\title{
Improving Finite-Volume Diffusive Fluxes Through Better Reconstruction
}

by

Chandan Balachandra Sejekan

B.Tech., Mechanical Engineering, National Institute of Technology Karnataka, Surathkal (India), 2013

A THESIS SUBMITTED IN PARTIAL FULFILLMENT OF

THE REQUIREMENTS FOR THE DEGREE OF

MASTER OF APPLIED SCIENCE

in

THE FACULTY OF GRADUATE AND POSTDOCTORAL STUDIES

(Mechanical Engineering)

THE UNIVERSITY OF BRITISH COLUMBIA

(Vancouver)

April 2016

(c) Chandan Balachandra Sejekan 2016 


\section{Abstract}

The overarching goal of CFD is to compute solutions with low numerical error. For finitevolume schemes, this error originates as error in the flux integral. For diffusion problems on unstructured meshes, the diffusive flux (computed from reconstructed gradients) is one order less accurate than the reconstructed solution. Worse, the gradient errors are not smooth, and so no error cancellation accompanies the flux integration, reducing the flux integral to zero order for second-order schemes. Our aim is to compute the gradient and flux more accurately at the cell boundaries and hence obtain a better flux integral for a slight increase in computational cost. We propose a novel reconstruction method and flux discretization to improve diffusive flux accuracy on cell-centred, isotropic unstructured meshes. Our approach uses a modified least-squares system to reconstruct the solution to second-order accuracy in the $\mathrm{H}_{1}$ norm instead of the prevalent $\mathrm{L}_{2}$ norm, thus ensuring second-order accurate gradients. Either circumcentres or containment centres are chosen as the control-volume reference points based on a criteria to facilitate calculation of secondorder gradients at flux quadrature points using a linear interpolation scheme along with a high-accuracy jump term to enhance stability of the system. Numerical results show a significant improvement in the order of accuracy of the computed diffusive flux as well as the flux integral. When applied to a channel flow advection-diffusion problem, the scheme resulted in an increased order of accuracy for the flux integral along with gains in solution accuracy by a factor of two. The characteristics of the new scheme were studied through stability, truncation error and cost analysis. The increase in computational costs were modest and affordable. The behaviour of the scheme was also tested by implementing a variation of it within the ANSYS Fluent discretization framework. 


\section{Preface}

The research ideas and methods discussed in this thesis are the fruits of a close working relationship between Dr. Carl Ollivier-Gooch and Chandan Balachandra Sejekan. The implementation of methods, the data analysis and the manuscript preparation were done by Chandan Balachandra Sejekan with invaluable guidance from Dr. Carl Ollivier-Gooch throughout the process. 


\section{Table of Contents}

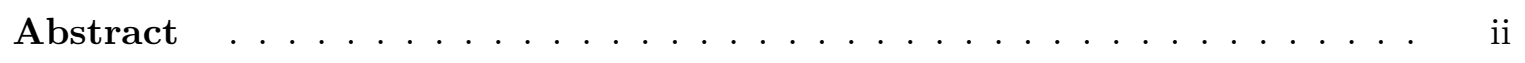

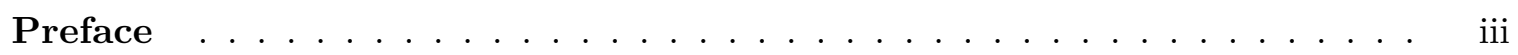

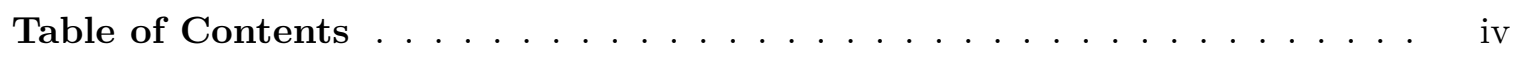

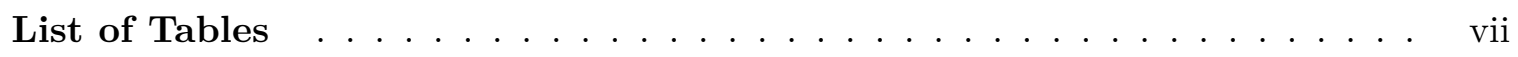

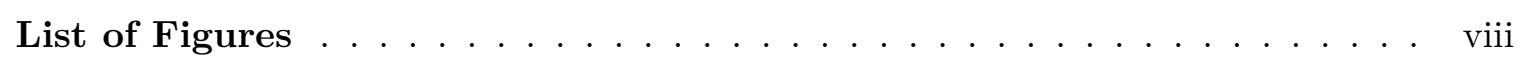

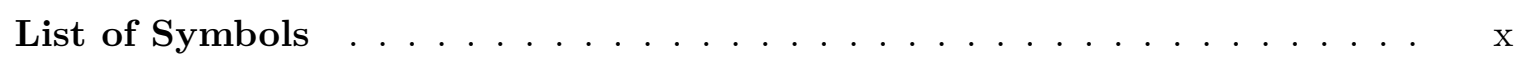

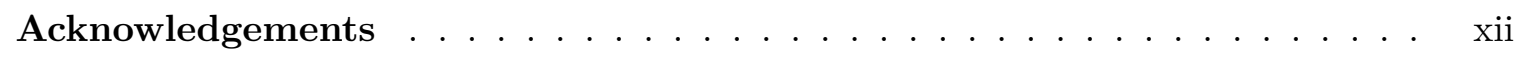

Dedication - The Master Voyage ............. xiii

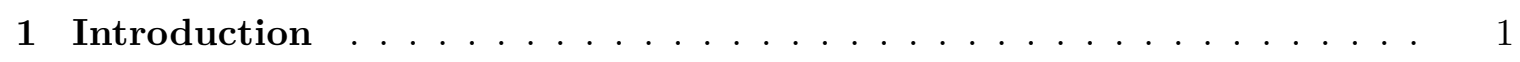

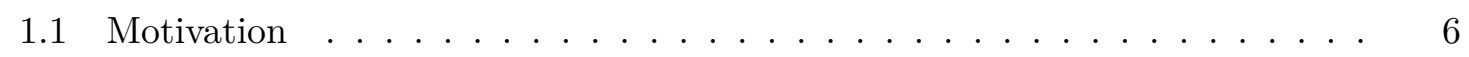

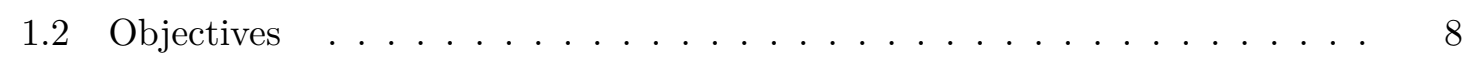

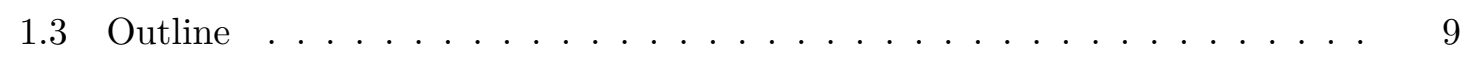

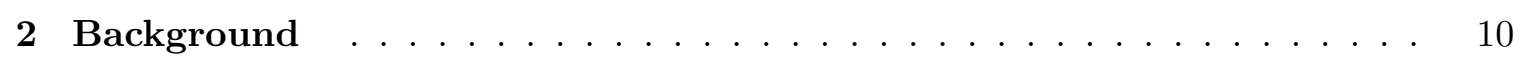

2.1 The Finite-Volume Solver . . . . . . . . . . . . . . . . . . 10

2.1 .1 Mesh Pre-processing . . . . . . . . . . . . . . . . . . 14

2.1.2 Solution Reconstruction . . . . . . . . . . . . . . . . 14

2.1 .3 Flux Evaluation . . . . . . . . . . . . . . . 21

2.1.3.1 Diffusive Flux Evaluation _. . . . . . . . . . 22

2.1.3.2 Face Gradients . . . . . . . . . . . 23 
2.1.3.3 Jump Terms . . . . . . . . . . . . . . . . . . . . . . 24

2.1.4 Flux Integration $\ldots \ldots \ldots \ldots \ldots$

2.1.5 Time Evolution _. . . . . . . . . . . . . . 26

2.2 Flux Error Reduction _. . . . . . . . . . . . . . . . . . . 27

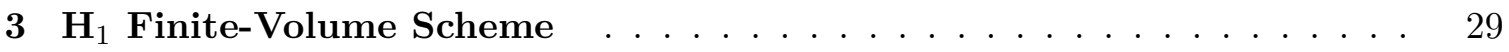

$3.1 \mathrm{H}_{1}$ Reconstruction . . . . . . . . . . . . . . . . . . . 29

$3.2 \mathrm{H}_{1}$ Flux Discretization $\ldots \ldots \ldots \ldots \ldots \ldots \ldots \ldots$

3.2.1 Theory in One Dimension _. . . . . . . . . . . . . 31

3.2.2 Extension to Two Dimensions _ . . . . . . . . . . . . . . . . 32

3.2.2.1 Circumcentres . . . . . . . . . . . . . 33

3.2.2.2 Containment Centres . . . . . . . . . . . 35

3.2.2.3 High-Accuracy Jump Term . . . . . . . . . . . . . . . . 36

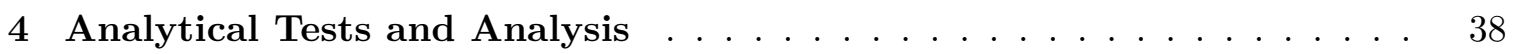

4.1 Methodology . . . . . . . . . . . . . . . . . . 38

4.2 Analytical Results . . . . . . . . . . . . . . . . . . 39

4.2.1 Linear Reconstruction . . . . . . . . . . . . . . . . . . . . . 39

4.2 .2 Quadratic Reconstruction . . . . . . . . . . . . . . 43

$4.2 .3 \quad \mathrm{H}_{1}$ Reconstruction . . . . . . . . . . . . . . . . . 45

4.2.3.1 Regular Stencil $\mathrm{H}_{1}$ Reconstruction . . . . . . . . 45

4.2.3.2 Extended Stencil $\mathrm{H}_{1}$ Reconstruction . . . . . . . . . . 47

5 Numerical Tests and Analysis . . . . . . . . . . . . . . . . 49

5.1 Preliminary Studies _. . . . . . . . . . . . . . . 50

5.1.1 One-dimensional $\mathrm{H}_{1}$ Flux Discretization . . . . . . . . . . . . 50

5.1 .2 Two-dimensional $\mathrm{H}_{1}$ Reconstruction Tests . . . . . . . . . 51

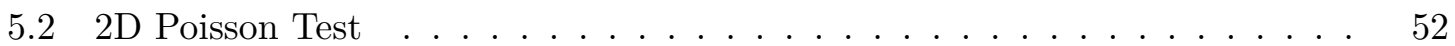

5.3 2D Advection-Diffusion Test . . . . . . . . . . . . . . . 57

6 Characterization Tests and Behavioural Analysis . . . . . . . . . . 61

6.1 Stability Analysis . . . . . . . . . . . . . . . . 61 
6.2 Truncation Error Analysis f . . . . . . . . . . . . . . 65

6.3 Cost Comparisons _. . . . . . . . . . . . . . . . . . . 69

$6.4 \mathrm{H}_{1}$ Finite-Volume Scheme in ANSYS Fluent Discretization . . . . . . . . 73

6.4.1 Fluent Diffusive Flux Discretization . . . . . . . . . . . . . . . 73

6.4.1.1 Reconstruction Gradients . . . . . . . . . . 73

6.4.1.2 Diffusion Gradients . . . . . . . . . . . . . . . 74

6.4.1.3 Diffusive flux formulation . . . . . . . . . . 75

6.4.1.4 Boundary Fluxes _. . . . . . . . . . . . . 76

$6.4 .2 \mathrm{H}_{1}$ Scheme in Fluent Diffusive Flux . . . . . . . . . . . . . . 76

6.4.3 Comparative Numerical Test Results _ . . . . . . . . . . . . 77

6.4.3.1 2D Poisson Test . . . . . . . . . . . . 78

6.4.3.2 2D Advection-Diffusion Test . . . . . . . . . . . . 80

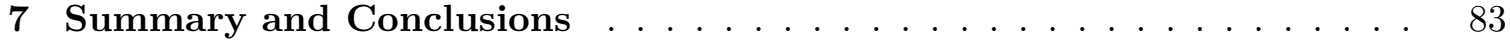

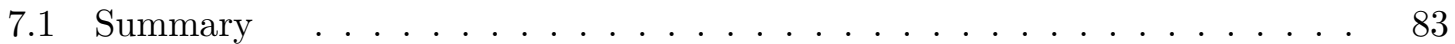

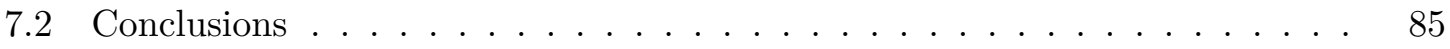

7.3 Recommended Future Work _. . . . . . . . . . . . . 87

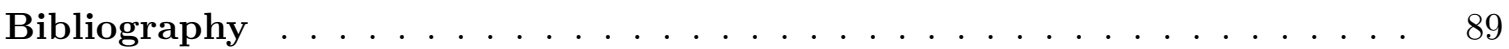




\section{List of Tables}

2.1 Flux Evaluation Strategies . . . . . . . . . . . . . . . . 25

2.2 Gauss-point locations . . . . . . . . . . . . . . 25

4.1 Stencils and truncation error for Laplacian on a cell-centred mesh . . . . . . 40

5.1 Orders of accuracy for Poisson model problem . . . . . . . . . . . . . 53

5.2 Orders of accuracy for advection-diffusion problem . . . . . . . . . 60

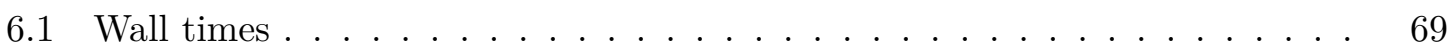




\section{List of Figures}

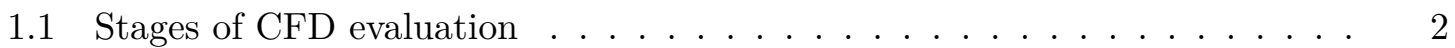

1.2 Mesh generation approaches over a two-dimensional airfoil . . . . . . . . . 3

1.3 Order of reconstruction accuracy of unstructured least-squares reconstruction schemes. ........................... 7

1.4 Solution error comparison of second and third order least-squares reconstruction 8

2.1 Control volume illustration f . . . . . . . . . . . . . . . . . . 11

2.2 High-order reconstruction stencil for cell centred mesh type . . . . . . . . . 17

2.3 Mesh geometrical features . . . . . . . . . . . . . . . . . . . 22

2.4 Gauss quadrature points schematic . . . . . . . . . . . . . 26

3.1 1D Non-uniform Grid Stencil . . . . . . . . . . . . . . . . . . . . 31

3.2 Various control-volume reference centres . . . . . . . . . . . . . . . 33

3.3 Circumcentre cases . . . . . . . . . . . . . . . . . . . . 34

3.4 The obtuse containment centre . . . . . . . . . . . . . . 36

4.1 Analytical Mesh coordinates and cell labels . . . . . . . . . . . . . . . 39

4.2 Second-order least-squares on uniform equilateral triangle mesh (decoupling) 42

4.3 Third-order least-squares on uniform equilateral triangle mesh . . . . . . . . 44

4.4 Regular $\mathrm{H}_{1}$ reconstruction on uniform equilateral triangle mesh (decoupling) 46

4.5 Extended $\mathrm{H}_{1}$ reconstruction on uniform equilateral triangle mesh (decoupling) 48

5.1 Isotropic unstructured meshes used . . . . . . . . . . . . . . . . 50

5.2 Preliminary Studies f . . . . . . . . . . . . . . . . . 51

5.3 Manufactured solutions for Poisson equation on an unstructured mesh . . . 52 
5.4 Case (i) Sinusoidal Poisson 2D test results . . . . . . . . . . . . . 54

5.5 Case (ii) Gaussian Bump Poisson 2D test results . . . . . . . . . . . . . 55

5.6 Case (iii) Plain Laplacian Poisson 2D test results . . . . . . . . . . . . . 56

5.7 Solution error comparison . . . . . . . . . . . . . . . . 57

5.8 Advection-diffusion solution $(\alpha=0.01) \ldots \ldots \ldots \ldots \ldots$

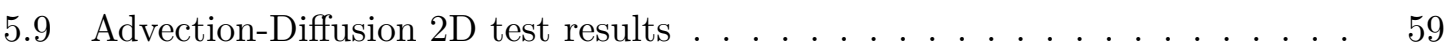

6.1 Eigenvalue plots - centroid vs. circumcentres . . . . . . . . . . . 62

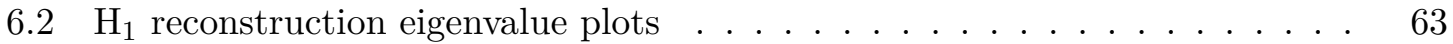

$6.3 \mathrm{H}_{1}$ reconstruction on equilateral mesh . . . . . . . . . . . . 64

6.4 Advention-diffusion eigenvalue plots . . . . . . . . . . . . 65

6.5 Truncation error convergence rate . . . . . . . . . . . 67

6.6 Truncation error magnitude plots $\ldots \ldots \ldots \ldots$. . . . . . 68

6.7 Jacobian calculation comparisons . . . . . . . . . . . . . 70

6.8 Comparison of total wall clock time required for convergence . . . . . . . 71

6.9 Flux error (steady state) vs total wall clock time required for convergence • 71

6.10 Time profile analysis . . . . . . . . . . . . . . . . . . . 72

6.11 Illustration of Fluent diffusive flux terms . . . . . . . . . . . . . . 75

6.12 Gradient stencils for diffusive fluxes about face $\mathrm{f} \ldots \ldots$. . . . . . . 76

6.13 Poisson solution convergence rates in Fluent software (Fluent-code) and Fluent discretization implementation in ANSLib (ANSLib-Fluent) $\ldots . . .78$

6.14 Poisson test results . . . . . . . . . . . . . . . . . . . . . 79

6.15 Advection-Diffusion solution convergence rates in Fluent software and Fluent discretization implementation in ANSLib . . . . . . . . . . . 81

6.16 Advection-Diffusion Solution convergence rates . . . . . . . . . . . . . 82 


\section{List of Symbols}

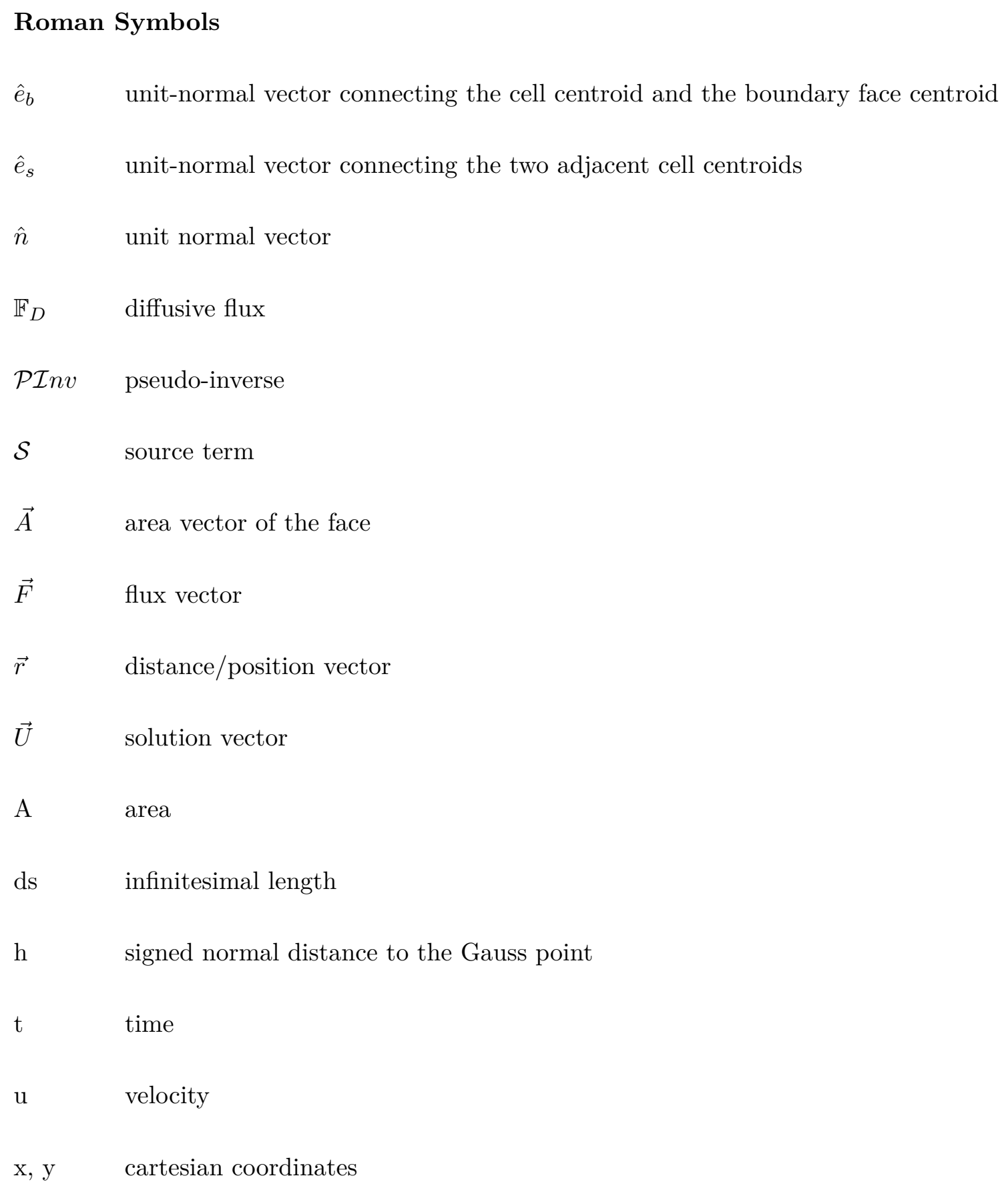




\section{Greek Symbols}

$\alpha \quad$ jump-term coefficient, Diffusion coefficient

$\epsilon \quad$ constant with a value of $10^{-9}$

$\eta \quad$ normal direction coordinate

$\Omega \quad$ size of control volume (area in 2D and volume in $3 \mathrm{D}$ )

$\bar{\phi} \quad$ control-volume-averaged value of the variable

$\phi \quad$ unknown variable, exact solution

$\widetilde{\phi} \quad$ reconstructed value of the variable

\section{Superscripts}

$+\quad$ left side evaluation

- $\quad$ right side evaluation

$\mathrm{m}, \mathrm{n} \quad$ polynomial exponents

\section{Subscripts}

2ndLS second-order least-squares reconstruction

3rdLS third-order least-squares reconstruction

CV control volume

g Gauss-point evaluation

$\mathrm{H}_{1} \quad$ proposed $\mathrm{H}_{1}$ reconstruction

i original control volume

j neighbouring control volume

L left CV reference point value

$\mathrm{R} \quad$ right $\mathrm{CV}$ reference point value 


\section{Acknowledgements}

I owe my gratitude to all those people who have made this thesis possible and because of whom my graduate experience has been one that I will cherish forever.

It is difficult to overstate my appreciation of my research supervisor Dr. Carl OllivierGooch, whose constant support, patience and guidance has been invaluable throughout the course of this research. I count myself lucky to have him as a supervisor and mentor. I have learned much from him not just in academics and research but also valuable life lessons in the virtues of patience, compassion and humility.

I would also like to acknowledge my colleagues at ANSLab research group Gary, Varun and Alireza for bearing with me and clarifying even the most trivial of doubts. Without their support, my progress would have been much slower. My thanks also go to Reza and Mahkame for proof-reading this document.

I am highly grateful to my friends for all the wonderful trips and fond memories. They are the spice that made my journey less humdrum.

Last but most importantly, I wish to thank my beloved family back in India for their unceasing, unwavering love and encouragement. They have been instrumental in moulding me into who I am today. It is to them that I dedicate my thesis. 


\section{The Master Voyage}

Rainy welcome to a city known anew.

Long arduous journey cast aside

With aspirations and zeal renew.

Stepping out boldly astride,

Across uncharted lands, uncharted life.

Friends from the past reckon.

Friends from the future beckon.

Friends galore together strive

Through highs and lows, a hive,

Over uncharted lands, uncharted life.

Homes of fun and laughter, incomplete

Without fond family far far away.

Constant craving to carve out a tunnel

Through the Earth's infernal core,

Through uncharted lands, uncharted life.

Travel and learning hand in hand.

New sights, hills, and mountains,

Knowledge to explore and surmount.

Aspiring to grow steadfast,

Learning uncharted lands, uncharted life.

Innumerable steps this voyage take.

Countless crowds cater this eager soul,

A morsel here, a feast there.

With all their unwavering support, I purport

Conquering uncharted lands, uncharted life.

- Chandan B. Sejekan

23rd March, 2016 


\section{Chapter 1}

\section{Introduction}

Computational Fluid Dynamics (CFD) has grown tremendously in the past decades to play a vital role in the study and design of fluid flows. Thanks to the accessibility of affordable high-performance computing hardware, CFD has become a mainstay in industries from civil construction to aerospace. It thus forms the third front in fluid analysis along with theoretical and experimental analysis. In fact CFD is preferred in the design fields due to its significant advantages of quick turn-around times, greater level of detail and flexibility as well as significantly lower costs of designs.

Most CFD analysis can be broken down to comprise of three basic stages of evaluation [57]; pre-processing, flow solver run and post-processing. We will briefly touch on each of these stages so as to better identify where the current research resides in the whole scheme of things. Figure 1.1 presents the same information in a schematic flow chart.

Pre-processing: The pre-processing stage prepares all of the necessary inputs that are required by the flow solver stage, in a format that can be understood by it. This stage essentially comprises of physical modelling and mesh generation. In the former, we mathematically define or model the physics of the flow problem that needs to be solved. This entails identification of the physical and chemical phenomena to be modelled and defining the geometry of the computational domain as well as its boundary conditions. We are essentially writing mathematical governing equations, typically in the form of partial differential equations (PDEs), that define the flow physics or approximate them. The error that arises due to this approximation of the flow physics is known as physical modelling error. This form of error varies greatly depending on the flow being analyzed.

The boundary conditions are equally important and should not be neglected. They directly influence the solution obtained at every stage. There are two common classifications 


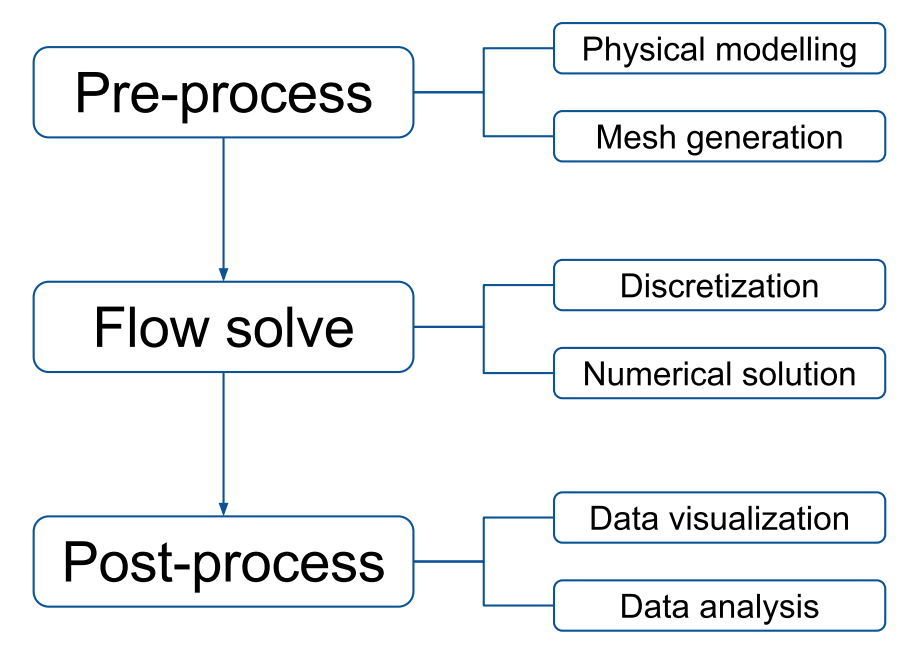

Figure 1.1: Stages of CFD evaluation

of boundary conditions: Dirichlet and Neumann. Dirichlet boundary conditions directly specify a value for the given variable at the boundary, for instance isothermal wall or constant velocity inlet conditions. Neumann conditions, on the other hand, specify a normal gradient for that variable at the boundary like the adiabatic wall or outlet conditions. The third and rare type, the Robin boundary condition is usually an expression which is a combination of the Dirichlet and Neumann boundary types. Its implementations can be found in convective heat transfer models.

Once the geometry of the computational domain has been defined, we can generate a mesh for it, which essentially involves decomposing the domain into finite non-overlapping sub-domains. These sub-domains are commonly known as cells or control volumes. The flow solver discretizes the governing equations on these individual sub-domains. Thus the finer these sub-domains are the more accurate the solution will be, while also increasing the computational costs. The mesh generation can follow two approaches, structured or unstructured. A structured mesh, as the name suggests, has a regular topology, which makes identification of each cell predictable and implicit. For instance the cell $(i, j, k)$ would always have $(i \pm 1, j \pm 1, k \pm 1)$ as its neighbours. This implicit cell connectivity makes it easier for the flow solver to process structured meshes. However, meshing of complex geometries is virtually impossible to automate for all possible geometries and thus demands a significant chunk of total CFD analysis time. In contrast unstructured meshes 


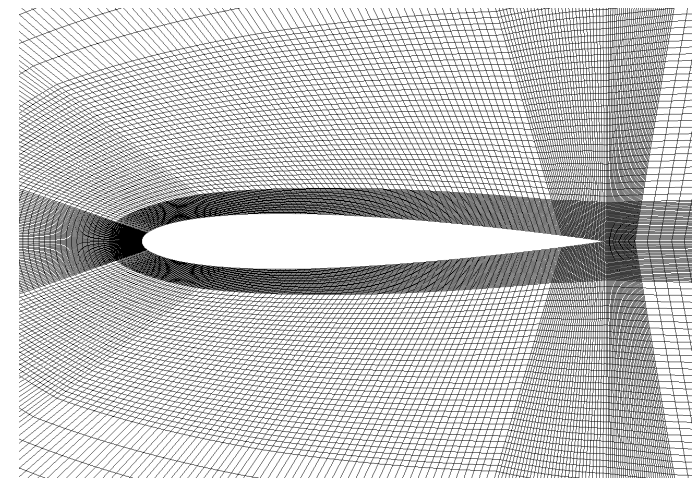

(a) Structured meshing

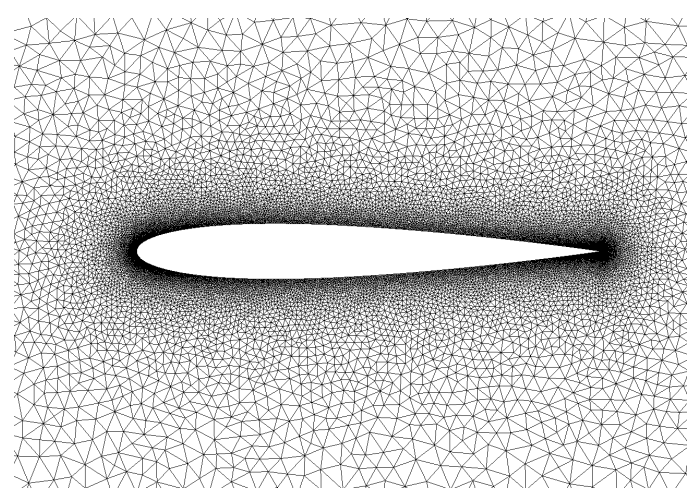

(b) Unstructured meshing

Figure 1.2: Mesh generation approaches over a two-dimensional airfoil

do not enforce a regular topology. This makes it much easier to mesh any geometry as an arbitrary combination of cells, commonly using triangles in two dimensions and tetrahedra in three dimensions. This assures flexibility and automation in meshing. However, now the cell connectivity needs to be explicitly defined which makes writing efficient unstructured flow solvers all the more challenging. Fortunately with advances with solver techniques, use of unstructured meshes has become common and proves to be more successful for complex problems [56]. These two approaches to mesh generation have been illustrated in Figure 1.2.

Flow Solve: With the mathematical model equations describing the flow field finalized, we need to discretize these PDEs within each cell, to enable their numerical approximation to the exact solution. A wide variety of discretization schemes and methods have been devised by researchers over the years. Broadly they fall under three main families; finitedifference, finite-element (and spectral element) and finite-volume schemes.

The common outline binding all three families is,

1. Discretize (approximate) the governing equations, in space and time if necessary, into a system of algebraic equations,

2. Solve these algebraic equations by some iterative method.

The approximation in the first step leads to the error known as the truncation error, which is the difference between the discrete algebraic equations and the continuous PDEs. The 
latter step results in the final numerical solution and an associated error known as the discretization error which can be defined as the difference between the exact solution of the governing equations and the numerical solution obtained from the scheme. As might be expected, the discretization error is related to the truncation error by the error transport equation, as shown by Roy [49]. Roy also highlighted the importance of truncation error for error estimation and also its applications in mesh adaptation to reduce the overall error [48]. The most common approach to reducing the discretization error to improve the numerical accuracy is either to use a finer mesh ( $h$-refinement) or to increase the order of accuracy ( $p$-refinement).

The finite-difference approach has historical value being the very first numerical technique to be developed for differential equations. It relies on using Taylor series expansions to approximate the point-wise values of the solution variables. It is simple and mathematically intuitive. However, conservation of variables needs to be explicitly handled; also its extension to unstructured meshes is quite a challenge. Excellent overviews of the finitedifference process can be found in the books $[2,27]$ with numerous works in the literature on advanced topics such as $[45,24]$.

The finite-element techniques finds its origins in the structural analysis of solids and was later applied to fluids. It encompasses methods such as the continuous Galerkin, streamlineupwind/Petrov-Galerkin (SUPG) $[9,16]$, discontinuous Galerkin [15, 10], spectral volume, spectral difference $[23,26]$ and more recently flux reconstruction $[17,58]$ methods. In these methods the solution is approximated by polynomial basis functions of the required degree within each cell, and the approximate solution is allowed to be discontinuous at the cell interfaces. Solution between neighbouring cells is coupled via the use of a common numerical flux applied at these cell interfaces. It is easily extended to unstructured meshes. However, the conservation of properties is still not guaranteed, and is particularly a challenge in flows with discontinuities such as shock waves.

The finite-volume scheme, which is at the heart of our research, takes the control-volume analysis approach commonly used in thermofluids systems. Here we integrate the conservative form of the governing equations over each control volume. This step distinguishes the finite-volume method from the others since the resulting numerical scheme is inher- 
ently conservative. This conservative property makes it easy to understand, intuitive and attractive for flows with discontinuities. Once again there are several schemes that fall under this umbrella such as the Green-Gauss, least-squares reconstruction (also known as

k-exact reconstruction), essentially non-oscillatory schemes (ENO) $[14,1]$ and its weighted modifications (WENO) $[25,12]$. A detailed description of the most commonly implemented finite-volume scheme, the least-squares technique, will follow in Chapter 2.

Post-processing: The final stage is the presentation and analysis of the numerical solution obtained. This generally involves visualization in the form of vector plots, surface plots, contour plots, and the like. There are innumerable plotting tools at one's disposal among which we found gnuplot, matplotlib (python) and Paraview to be useful for us.

\section{Software Frameworks Used}

The Advanced Numerical Simulations Library (ANSLib) [41], a CFD tool developed and maintained at the Advanced Numerical Simulations Laboratory (ANSLab) in the University of British Columbia, Vancouver, was used as the primary test bed in this research. It is an unstructured finite-volume flow solver that is capable of handling two and three dimensional meshes with accuracies ranging from first to fourth order for a variety of flow physics problems.

All the meshes used in this research were generated using the meshing software, Generation and Refinement of Unstructured Mixed-element Meshes in Parallel (GRUMMP) [40] also developed at ANSLab, UBC. It is a general purpose automatic mesh generation software for unstructured meshes with mixed element types in two and three dimensions. The software can produce high-quality meshes that meet user-defined mesh density requirements, using elements appropriate for the geometry and physics of a particular problem. It also supports mesh refinement, optimization, coarsening and local remeshing. 


\subsection{Motivation}

First and second order finite-volume schemes (commonly known as low order) are widely utilized in the industrial and commercial Computational Fluid Dynamics (CFD) codes for their robustness and algorithm simplicity. However they fail to satiate the ever increasing demands of accuracy expected from CFD algorithms. High-order methods (having a spatial accuracy of third-order or higher) can help meet this demand for accuracy. Various researchers at the First (2012), Second (2013) and Third (2015) International Workshops on High-Order CFD Methods suggest better performance and accuracy compared to second-order methods with lower computational costs for the same target solution error $[5,51,59,18]$. High-order schemes thus show great promise. Yet industry level adoption of high-order accurate schemes has been slow. This may be attributed to the increased algorithmic complexity and less robust behaviour of high-order schemes. There has been conscientious effort by the high-order community over the years to address this issue and bridge the current chasm that exists between the technology being driven in academia and the current algorithms adopted in practical applications.

High-order finite-volume methods, presented in section 2.1, are in a unique position to answer this calling since they are inherently an extension of the second-order finitevolume methods currently utilized in the industry. This significant advantage should make their wide-spread adoption easier. Unfortunately they do demand a substantial increase in computational resources per control volume and also a greater algorithmic complexity. This has proved to be a major deterrent in their adoption. We hope to mitigate this situation with the development of an intermediate finite-volume scheme that combines the best of both worlds, while being an easy upgrade from the existing second-order scheme.

It is well known among the CFD community that a p-order-accurate solution reconstruction would produce a p-order-accurate convective flux that depends only on the solution. However diffusive fluxes, since they also depend on the gradient, turn out to be of only (p-1)-order-accuracy. This can be visualized in Figure 1.3a, for which we set up a secondorder finite-volume least-squares reconstruction for an unstructured mesh (details follow in Chapter 2) and plotted the respective orders of accuracy of the reconstructed solution, 


\subsection{Motivation}

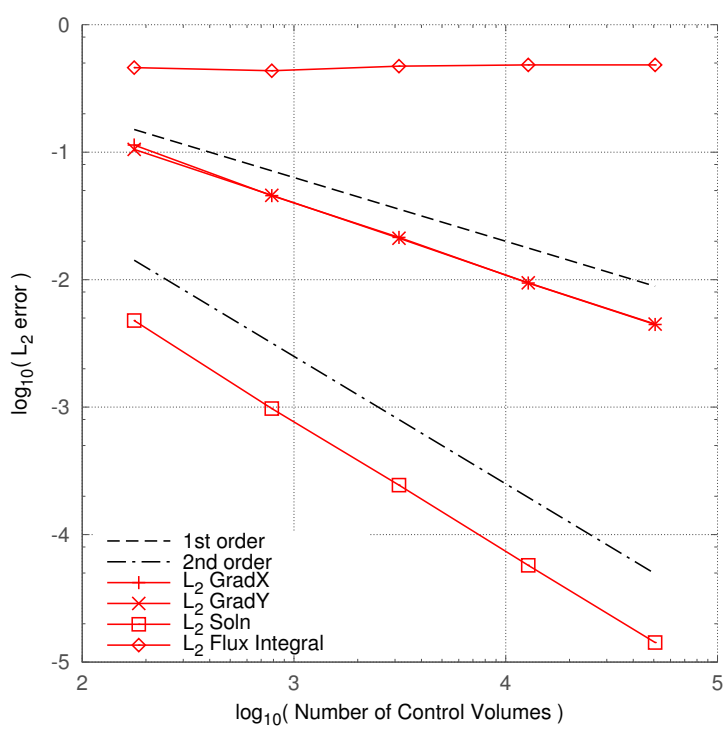

(a) Second-Order

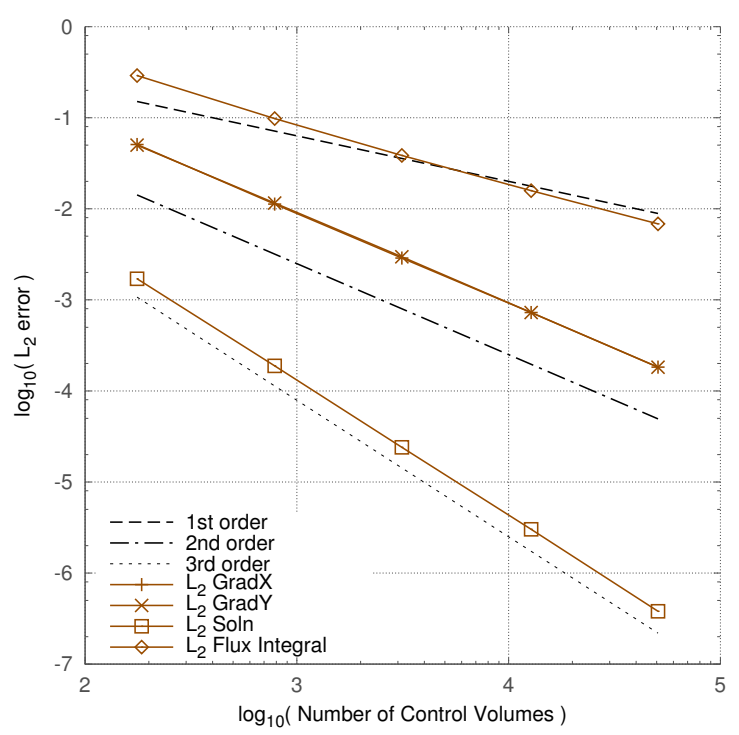

(b) Third-order

Figure 1.3: Order of reconstruction accuracy of unstructured least-squares reconstruction schemes.

the gradient and the flux integral. We can observe that although the solution is indeed second-order as anticipated, the gradient reconstruction is one order less accurate while the flux integral is even worse at zero-order. A similar trend can be seen in the convergence rate of a third-order scheme (Figure 1.3b), with loss of an order of accuracy as we move from solution to gradient to flux-integral. We aim to address this shortcoming of the flux with our research.

Any improvements in the accuracies of the flux and gradients would be beneficial in various applications where the physical quantities rely on the gradient evaluation such as viscous stress and heat fluxes in the Navier-Stokes equations as well as the gradient-based source terms that are used in many turbulence flow models such as the Spalart-Almaras turbulence model.

It is also reasonable to expect that any improvement in the flux would ultimately translate into improvements in the flux integral as well. This in turn should cascade into improvements in the truncation error, which is known to be identical to the flux integral obtained from smooth initial data. This would entail improvements in all of the areas where truncation error estimation plays a vital role namely mesh adaptation [48], error estimation[49], 


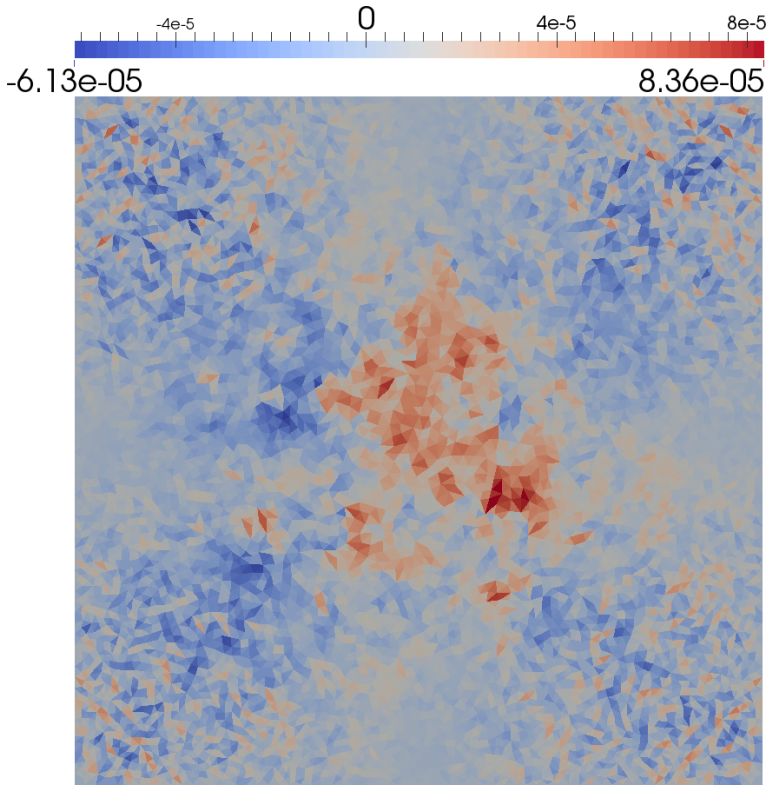

(a) Second-order solution error

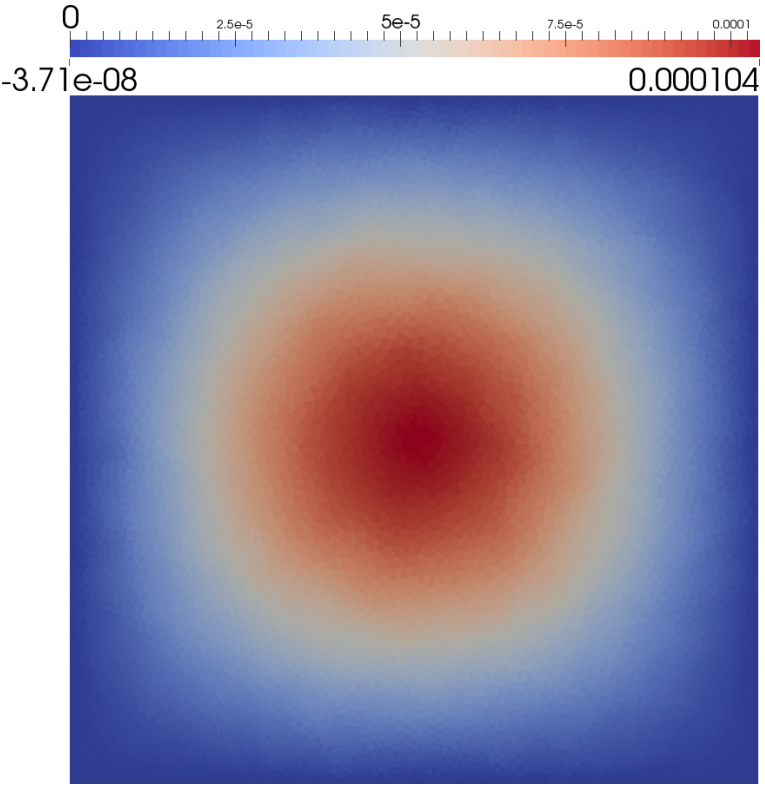

(b) Third-order solution error

Figure 1.4: Solution error comparison of second and third order least-squares reconstruction

defect correction as well as the adjoint problem [13]. Section 6.2 will further touch on this topic.

Moreover, as Figure 1.4 shows, the error characteristics of the solution also vary greatly with order of accuracy. The second-order and the third-order least-squares schemes show a striking difference in the smoothness of the solution discretization and truncation errors, with the latter providing remarkably smoother errors. As can be observed, the smoother solution error also reflects the shape of the underlying solution, which in this case was $\frac{\pi}{8} \sin (\pi x) \sin (\pi y)$. A smoother solution error enables better application of various error estimation algorithms [50].

\subsection{Objectives}

The primary goal of this research is to develop a finite-volume framework that would improve the diffusive fluxes to an order of accuracy on par with the solution order of accuracy.

In particular we will focus on obtaining second-order accurate diffusive fluxes at the cell interfaces especially at points of interest known as Gauss points at which the flux values are integrated using Gauss quadrature to compute the flux integral. 
We also aim to achieve this at a modest computational cost which in this context should be between the cost required for a full second-order finite-volume least-squares scheme and third-order finite-volume scheme.

Essentially as an general guideline, we strive to strike a balance between the best of second and third order finite-volume schemes.

Once developed we will look at evaluating and analyzing the characteristics of the new numerical scheme for important qualities of robustness (or in other words, stability) as well as the truncation error generated.

\subsection{Outline}

We begin by laying down a detailed foundation of the general finite-volume flow solver currently implemented in ANSLib in Chapter 2. We will focus on key aspects of the solver including the solution reconstruction and the flux discretization, which are most relevant to this research, while briefly discussing the rest namely the flux integration and the time advance techniques of the solver.

Chapter 3 provides details of the proposed new finite-volume scheme which we have named the $\mathrm{H}_{1}$ finite-volume scheme, so called because our solution reconstruction is second order accurate in the $\mathrm{H}_{1}$ norm.

Analytical tests were conducted, the results of which are presented in Chapter 4. We juxtapose the new scheme with the second and third order finite-volume least-squares schemes and draw inferences. The analytical tests are only practical for a uniform equilateral triangular mesh, while the numerical tests are more versatile and give a better picture.

Chapter 5 highlights the results of the numerical tests and once again the $\mathrm{H}_{1}$ finitevolume scheme is compared with the other two finite-volume schemes. We explore the characteristics and behaviour of the new scheme in Chapter 6, focusing on the traits of stability, truncation error and computational cost. Finally, we conclude with Chapter 7, wherein we summarize, provide some concluding remarks and also list possible avenues for future research and development. 


\section{Chapter 2}

\section{Background}

The relevant CFD practices that are essential for the understanding of the current research will be expounded in this chapter. The Advanced Numerical Simulations Library (ANSLib), the unstructured finite-volume flow-solver developed at the Advanced Numerical Simulations Lab at UBC, has been the primary test bed for this research. We begin with some insight into the workings of a finite-volume flow-solver with emphasis on the solution reconstruction and flux evaluation followed by an overview of flux integral evaluation and time advance techniques. We will then move on to an understanding of error reduction in subsequent sections of this chapter.

\subsection{The Finite-Volume Solver}

Finite-volume methods have long been recognized as one of the most robust and reliable discretization techniques available in CFD. As mentioned earlier, these methods inherently utilize the conservative form of the equations that govern fluid mechanics and as such require the physical domain to be divided into a number of control volumes. There are various approaches to forming the individual control volumes based on a mesh but the most commonly implemented are cell-centred and vertex-centred.

Cell-centred control volumes are formed directly from the underlying mesh grid with the cells of the mesh defined as the control volumes. In this approach the cell centroid is commonly chosen as the cell reference point for the cell, serving as the local origin for a reconstruction polynomial described later in this chapter. The vertex-centred approach, on the other hand, directly utilizes the mesh vertices as the cell reference points and builds control volumes around them, typically using the median-dual as the cell perimeter. The median-dual is defined as the lines connecting the cell centroids and edge midpoints. 


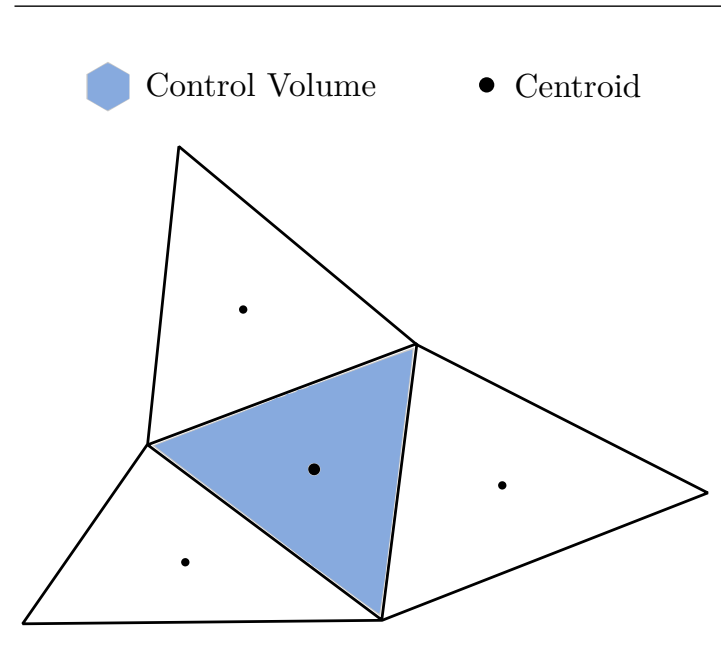

(a) Cell centered control volume

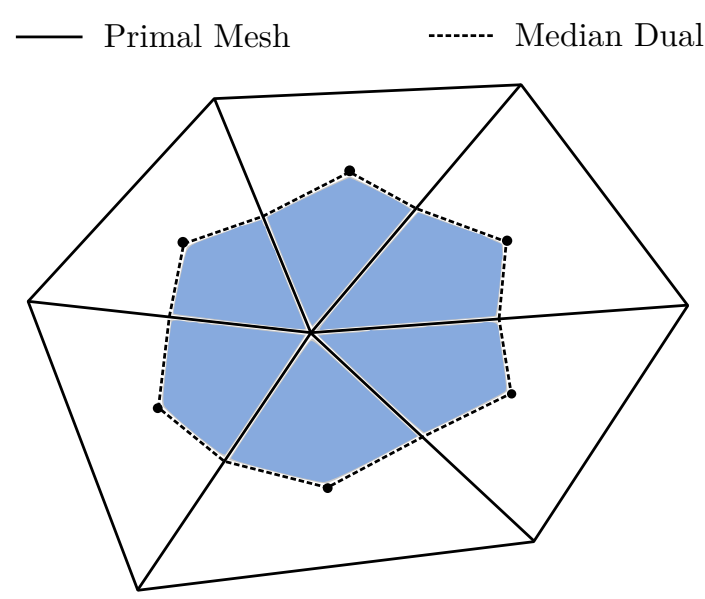

(b) Vertex centered control volume

Figure 2.1: Control volume illustration

Figure 2.1 provides a graphical illustration of the two common types of control volumes. Both forms have their inherent advantages and disadvantages. An inquisitive reader is directed to the work of Diskin et al. [11] for an in depth comparison of the two. In our research we have primarily focused on the two-dimensional cell-centred unstructured mesh domain and shall restrict our discussion to the same.

The conservative form of the fluid governing equations can be cast into a generic form [2] which can be written as

$$
\frac{\partial \vec{U}}{\partial t}+\nabla \cdot \vec{F}=\overrightarrow{\mathcal{S}}
$$

where $\vec{U}$ denotes the solution vector, $\vec{F}$ the flux dyad and $\overrightarrow{\mathcal{S}}$ the source term. In general our flow solver ANSLib works with the dimensionless form of the governing equations and hence in this thesis all the governing equations are cast in the dimensionless form.

Taking the integral of Equation 2.1 over an arbitrary control volume $C V_{i}$ and utilizing the divergence theorem we obtain a $2 \mathrm{D}$ finite-volume formulation of the governing equations as

$$
\iint_{C V_{i}} \frac{\partial \vec{U}}{\partial t} d A+\oint_{\partial C V_{i}} \vec{F} \cdot \hat{n} d s=\iint_{C V_{i}} \overrightarrow{\mathcal{S}} d A
$$

where $\hat{n}$ is the outward unit normal vector and $d s$ is the infinitesimal for the line integral along the control volume edges. Put simply, this equation says that the rate of change of the unknown variable within a control volume should be equal to the net flux that crosses 
all its boundaries. This concept lies at the very heart of the finite-volume method.

Equation 2.2 can be further simplified upon assuming the discretized physical domain to be constant. The solution vector $\vec{U}$ can then be extracted as its control volume average. Thus we have

$$
\frac{d \overline{U_{i}}}{d t}+\frac{1}{A_{i}} \oint_{\partial C V_{i}} \vec{F} \cdot \hat{n} d s=\frac{1}{A_{i}} \iint_{C V_{i}} \overrightarrow{\mathcal{S}} d A
$$

where $A_{i}$ is the area of the control volume. The first term on the left-hand side is the time derivative of the averaged solution vector $\bar{U}$ and the second term is known as the flux integral or the residual, representing the spatial discretization of the control volume. The right hand side is the source term integral which is usually added on to the flux integral before advancing the solution vector in time.

For the purpose of our research, the steady-state two-dimensional Poisson equation

$$
\nabla^{2} \phi=\left(\frac{\partial^{2} \phi}{\partial x^{2}}+\frac{\partial^{2} \phi}{\partial y^{2}}\right)=\mathcal{S}
$$

is used as the model diffusion equation, where $\phi$ is the unknown scalar variable and $\mathcal{S}$ is again the source term. Here and throughout this thesis we will represent the unknown variable by $\phi$. The Poisson equation is a model for the steady-state diffusion equation and hence it appears in various forms within the fluid physics given by the Navier-Stokes equations. An unsteady form of the steady-state diffusion equation can be written as

$$
\frac{\partial \phi}{\partial t}-\left(\frac{\partial^{2} \phi}{\partial x^{2}}+\frac{\partial^{2} \phi}{\partial y^{2}}\right)=\mathcal{S}
$$

giving us the solution and flux vectors as

$$
\vec{U}=\phi ; \vec{F}=\left[\begin{array}{c}
-\frac{\partial \phi}{\partial x} \\
-\frac{\partial \phi}{\partial y}
\end{array}\right]=-\nabla \phi
$$

Applying a similar procedure as above we obtain the finite-volume formulation of the 
Poisson equation

$$
\frac{d \overline{\phi_{i}}}{d t}-\frac{1}{A_{i}} \oint_{\partial C V_{i}} \nabla \phi \cdot \hat{n} d s=\frac{1}{A_{i}} \iint_{C V_{i}} \mathcal{S} d A
$$

This form can now be implemented and solved for by a finite-volume flow solver. The overall finite-volume numerical scheme can be summarized in the following iteration stages [3]:

1. Mesh Pre-processing: The physical grid discretization is pre-processed to ensure consistency, define the control volumes as required and precompute a few geometric terms.

2. Solution Reconstruction: In the most simplified terms, the process of obtaining the piecewise polynomial coefficients for use in evaluating the fluxes in Equation 2.3 from the control volume averages for each cell, is known as solution reconstruction.

3. Flux evaluation: The numerical flux at individual Gauss-points are evaluated using the reconstructed solution.

4. Flux integration: The flux contributions in each cell are integrated by performing a high-order accurate flux quadrature along each edge, generally by Gaussian quadrature.

5. Time evolution: The solution is evolved in time using an adequate time stepping scheme such as Implicit Euler, Runge-Kutta etc., resulting once again in a new collection of cell averages.

6. Repeat stages 2 through to 5 until a steady-state or a time-bound solution is obtained.

Our current research presented in this thesis has its roots in the second and third stages of the finite-volume iteration laid out above. Hence, in this background discussion we will focus primarily on the same with a brief introduction to the other steps to provide a more holistic understanding. 


\subsubsection{Mesh Pre-processing}

Although not an integral component of the finite-volume numerical scheme, the mesh preprocessing stage is nonetheless essential from the computational point of view since it is in this stage that we analyze and process the physical domain discretization, more commonly known as the mesh.

The various control volumes are formed in this stage and also their respective geometric terms such as their areas, their cell reference point locations etc. are evaluated. The geometric moments are also precomputed and stored to be used later during the solution reconstruction stage.

This pre-processing is only performed once at the very beginning in the flow solver and as such does not demand much computational time.

\subsubsection{Solution Reconstruction}

Solution reconstruction is the primary, as well as the most complex, component of the finite-volume method [3]. Reconstruction provides an accurate estimate of the solution and gradients of the unknown variable, which is crucial since the flux integral in turn is derived from those very estimates and hence reconstruction ultimately limits the spatial accuracy of the finite-volume solution.

Numerous approaches to perform solution reconstruction have been developed and implemented over the years [8]. The second-order Green-Gauss method laid out by Barth [7] had been widely accepted by the CFD community for its simplicity and robustness. Barth $[6$, $3,4]$ also presented a high-order (loosely defined as third-order or more) approach in the form of k-exact reconstruction (also known as minimum energy or least-squares reconstruction) capable of $(\mathrm{k}+1)$ th-order accuracy.

The latter has been shown to be more accurate and stable with mean constraints and utilizing weighted reconstructions. The research of Ollivier-Gooch and Van Altena [43] extended this technique with boundary constraints in their finite-volume scheme for the advection-diffusion equation. Their least-squares reconstruction scheme is described in this section. We will, in Chapter 3, propose novel modifications to this technique for our research. 
We begin by fitting a piecewise polynomial representation of the solution within each control volume using a Taylor series expansion about the cell reference point $\left(x_{i}, y_{i}\right)$.

$$
\begin{aligned}
\widetilde{\phi}_{i}(x, y)= & \left.\phi\right|_{i}+\left.\frac{\partial \phi}{\partial x}\right|_{i}\left(x-x_{i}\right)+\left.\frac{\partial \phi}{\partial y}\right|_{i}\left(y-y_{i}\right) \\
& +\left.\frac{\partial^{2} \phi}{\partial x^{2}}\right|_{i} \frac{\left(x-x_{i}\right)^{2}}{2}+\left.\frac{\partial^{2} \phi}{\partial y^{2}}\right|_{i} \frac{\left(y-y_{i}\right)^{2}}{2} \\
& +\left.\frac{\partial^{2} \phi}{\partial x \partial y}\right|_{i}\left(x-x_{i}\right)\left(y-y_{i}\right)+\ldots
\end{aligned}
$$

where $\widetilde{\phi}_{i}$ is the reconstructed solution at any point $(x, y)$ and $\frac{\partial^{k+l} \phi_{i}}{\partial x^{k} \partial y^{l}}$ are its derivatives at the reference point $\left(x_{i}, y_{i}\right)$ of the control volume $i$. The solution and its derivatives are collectively known as the coefficients of the Taylor polynomial.

The eventual order of accuracy that the numerical scheme will be able to achieve is dictated by the number of derivatives evaluated in this Taylor series polynomial. Thus the higher the degree of this polynomial the greater will be the order of accuracy of the solution and truncating the polynomial to a degree $\mathrm{k}$ will result in at most $(\mathrm{k}+1)$ th-order accuracy of the scheme. For instance, truncating Equation 2.8 to just the first derivatives in $\mathrm{x}$ and $\mathrm{y}$ and thus obtaining a linear polynomial, will result in a second-order accurate reconstruction.

$$
\widetilde{\phi}_{i}(x, y)=\left.\phi\right|_{i}+\left.\frac{\partial \phi}{\partial x}\right|_{i}\left(x-x_{i}\right)+\left.\frac{\partial \phi}{\partial y}\right|_{i}\left(y-y_{i}\right)+\mathcal{O}\left(\Delta x^{2}, \Delta y^{2}\right)
$$

The coefficients of the Taylor polynomial are evaluated while conserving the mean value of the solution in the given control volume and also such that the reconstruction closely approximates the nearby neighbours' control volume averages. This is a task best performed by casting the system as a least-squares problem. Hence this scheme is known as leastsquares reconstruction $[38,39]$.

Conservation of the mean is an essential requirement of the finite-volume method. To ensure this, a mean constraint equation is included in the system before solving. For the 
reconstructed control volume this is given by

$$
\bar{\phi}_{i}=\frac{1}{A_{i}} \iint_{V_{i}} \widetilde{\phi}_{i}(x, y) d A
$$

Upon substituting for the reconstructed value from Equation 2.8 we note that

$$
\bar{\phi}_{i}=\left.\phi\right|_{i}+\left.\frac{\partial \phi}{\partial x}\right|_{i} \bar{x}_{i}+\left.\frac{\partial \phi}{\partial y}\right|_{i} \bar{y}_{i}+\left.\frac{\partial^{2} \phi}{\partial x^{2}}\right|_{i} \frac{\bar{x}_{i}^{2}}{2}+\left.\frac{\partial^{2} \phi}{\partial x \partial y}\right|_{i} \overline{x y}_{i}+\left.\frac{\partial^{2} \phi}{\partial y^{2}}\right|_{i} \frac{\bar{y}_{i}^{2}}{2}+\ldots
$$

where the geometric moments have been represented as

$$
{\overline{x^{m} y^{n}}}_{i}=\frac{1}{A_{i}} \iint_{V_{i}}\left(x-x_{i}\right)^{m}\left(y-y_{i}\right)^{n} d A
$$

In addition to conserving the mean of the reconstructed control volume, we also need to minimize the error in reconstructing the mean of the neighbouring control volumes. This empowers us with more equations that we can solve to obtain up to the $k^{\text {th }}$ derivative of Equation 2.8 as demanded by the reconstruction accuracy we seek. It also ensures that the reconstruction is relevant globally across the physical domain. A specific set of neighbours is chosen for each control volume which forms its reconstruction stencil $\left\{V_{j}\right\}_{i}$. The number of neighbours and the neighbours themselves that make up this reconstruction stencil are chosen based on the required reconstruction accuracy and their topological distance from the reconstructed cell, as illustrated in Figure 2.2.

Selecting control volumes that are physically close to the given cell is known as compact support [32]. Compact support has been shown to improve stability by emphasizing data that should heavily influence the reconstruction. Neighbours are thus added onto the reconstruction stencil until an over-determined least-squares system is created wherein the number of equations is more than the unknown coefficients for the desired order of accuracy. Thus for second-order accuracy just three neighbours are sufficient while third and fourth order accuracy demand at least six and ten neighbours respectively.

In practice however the stencils are precomputed by appending an entire layer of neigh- 


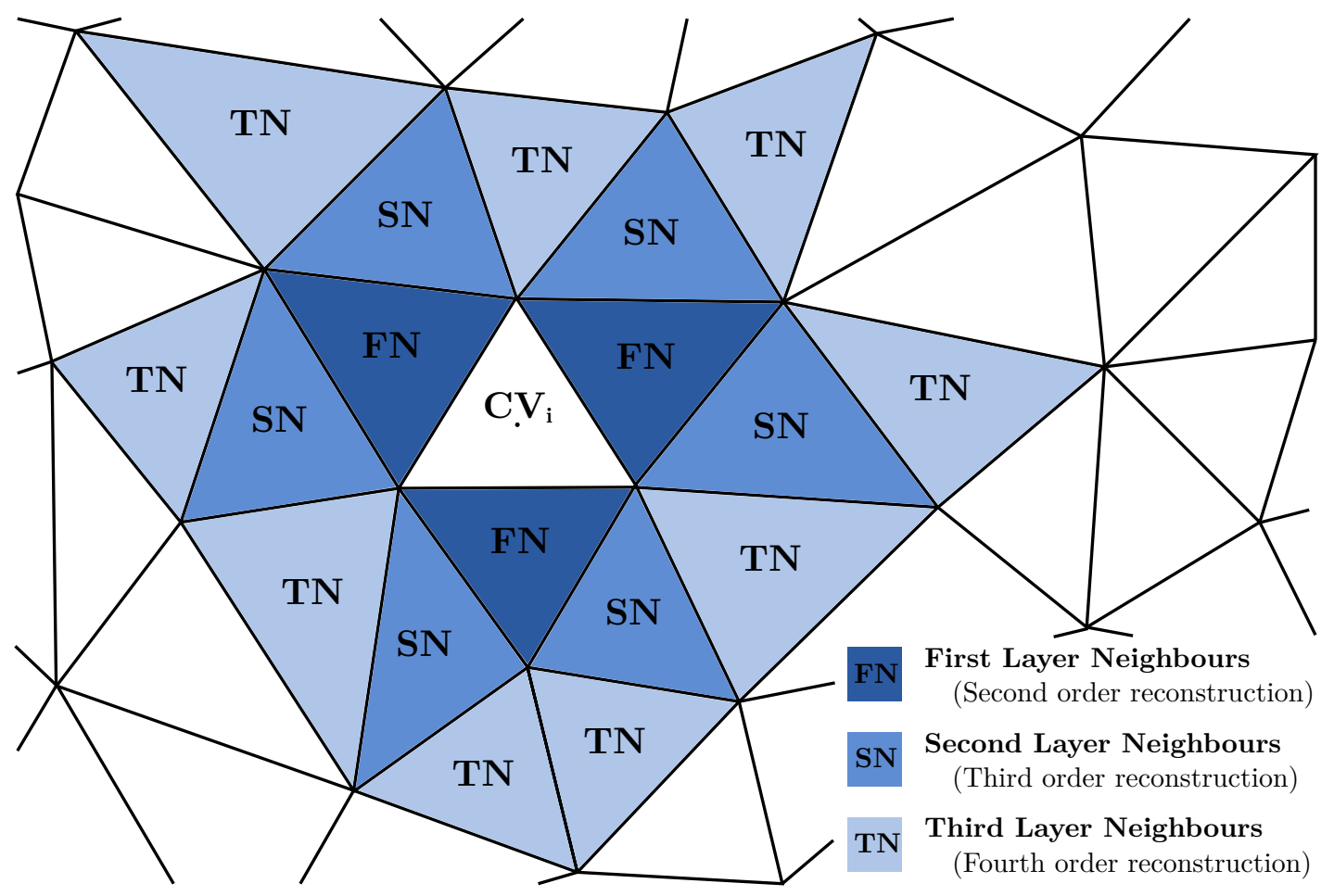

Figure 2.2: High-order reconstruction stencil for cell centred mesh type

bours to the stencil for each increase in order of accuracy such that the requirements are always met with an excess. When implementing for the cell centred mesh type, layers of neighbours are formed based on whom they share an edge with. The first neighbours are those that share a common edge with the given control volume, second neighbours in turn share a common edge with the first neighbours and so on. This generally results in stencil sizes of at least 3, 9 and 18 neighbours for second, third and fourth order accuracy. Doing so makes the system more robust and less susceptible to noise and non-smooth or oscillatory data.

Thus for every neighbouring control volume $V_{j}$ in the reconstruction stencil we need to satisfy as best we can a mean constraint for its control volume average. Mathematically this can be written as

$$
\bar{\phi}_{j} \approx \frac{1}{A_{j}} \iint_{V_{j}} \widetilde{\phi}_{i}(x, y) d A
$$

As before, expanding the right hand side using the Taylor series expansion of Equa- 
tion 2.8 we have

$$
\begin{aligned}
\frac{1}{A_{j}} \iint_{V_{j}} \widetilde{\phi}_{i}(x, y) d A= & \left.\phi\right|_{i}+\left.\frac{\partial \phi}{\partial x}\right|_{i}\left\{\frac{1}{A_{j}} \int_{V_{j}}\left(x-x_{i}\right) d A\right\}+\left.\frac{\partial \phi}{\partial y}\right|_{i}\left\{\frac{1}{A_{j}} \int_{V_{j}}\left(y-y_{i}\right) d A\right\} \\
& +\left.\frac{\partial^{2} \phi}{\partial x^{2}}\right|_{i}\left\{\frac{1}{2 A_{j}} \int_{V_{j}}\left(x-x_{i}\right)^{2} d A\right\}+\left.\frac{\partial^{2} \phi}{\partial y^{2}}\right|_{i}\left\{\frac{1}{2 A_{j}} \int_{V_{j}}\left(y-y_{i}\right)^{2} d A\right\} \\
& +\left.\frac{\partial^{2} \phi}{\partial x \partial y}\right|_{i}\left\{\frac{1}{A_{j}} \int_{V_{j}}\left(x-x_{i}\right)\left(y-y_{i}\right) d A\right\}+\ldots
\end{aligned}
$$

To simplify the moment calculation of every neighbouring control volume about the reference point of control volume $i$, we replace $\left(x-x_{i}\right)$ with $\left(x-x_{j}\right)+\left(x_{j}-x_{i}\right)$ and $\left(y-y_{i}\right)$ with $\left(y-y_{j}\right)+\left(y_{j}-y_{i}\right)$. Doing so effectively eliminates the otherwise complicated moment calculation by utilizing the known moments of each control volume about their own reference points. These geometrical moments of every cell can easily be computed in advance and stored during the initial stage of mesh pre-processing.

$$
\begin{aligned}
& \frac{1}{A_{j}} \iint_{V_{j}} \widetilde{\phi}_{i}(x, y) d A=\left.\phi\right|_{i}+\left.\frac{\partial \phi}{\partial x}\right|_{i}\left(\bar{x}_{j}+\left(x_{j}-x_{i}\right)\right)+\left.\frac{\partial \phi}{\partial y}\right|_{i}\left(\bar{y}_{j}+\left(y_{j}-y_{i}\right)\right) \\
& +\left.\frac{\partial^{2} \phi}{\partial x^{2}}\right|_{i}\left(\frac{\bar{x}_{j}+2 \bar{x}_{j}\left(x_{j}-x_{i}\right)+\left(x_{j}-x_{i}\right)^{2}}{2}\right) \\
& +\left.\frac{\partial^{2} \phi}{\partial x \partial y}\right|_{i}\left(\overline{x y}_{j}+\bar{x}_{j}\left(y_{j}-y_{i}\right)+\bar{y}_{j}\left(x_{j}-x_{i}\right)+\left(x_{j}-x_{i}\right)\left(y_{j}-y_{i}\right)\right) \\
& +\left.\frac{\partial^{2} \phi}{\partial y^{2}}\right|_{i}\left(\frac{\bar{y}_{j}+2 \bar{y}_{j}\left(y_{j}-y_{i}\right)+\left(y_{j}-y_{i}\right)^{2}}{2}\right)+\ldots
\end{aligned}
$$

These mesh dependent geometric moments can be generalized in the form

$$
\begin{aligned}
{\widehat{x^{m} y^{n}}}_{i j}= & \frac{1}{A_{j}} \iint_{V_{j}}\left(\left(x-x_{j}\right)+\left(x_{j}-x_{i}\right)\right)^{m} \cdot\left(\left(y-y_{j}\right)+\left(y_{j}-y_{i}\right)\right)^{n} d A \\
& =\sum_{l=0}^{n} \sum_{k=0}^{m} \frac{n !}{l !(n-l) !} \frac{m !}{k !(m-k) !}\left(x_{j}-x_{i}\right)^{k} \cdot\left(y_{j}-y_{i}\right)^{l} \cdot \overline{x^{m-k} y^{n-l}} j
\end{aligned}
$$

Thus the error minimization equation for the control volume averages of every neighbour 
within the stencil of the control volume $i$, can be written as

$$
\left.\bar{\phi}_{j} \approx \phi\right|_{i}+\left.\frac{\partial \phi}{\partial x}\right|_{i} \widehat{x}_{i j}+\left.\frac{\partial \phi}{\partial y}\right|_{i} \widehat{y}_{i j}+\left.\frac{\partial^{2} \phi}{\partial x^{2}}\right|_{i} \frac{\widehat{x}^{2}}{2}+\left.\frac{\partial^{2} \phi}{\partial x \partial y}\right|_{i} \widehat{x y}_{i j}+\left.\frac{\partial^{2} \phi}{\partial y^{2}}\right|_{i}{\widehat{y^{2}}}_{i j}+\ldots
$$

Hence the final least-squares system is formed for every control volume by collating together all these neighbouring error minimization equations. This system is also formed for each unknown variable in all the control volumes. The least-squares system, corresponding to the general form of $\mathbf{A} x=\beta$, can be written as

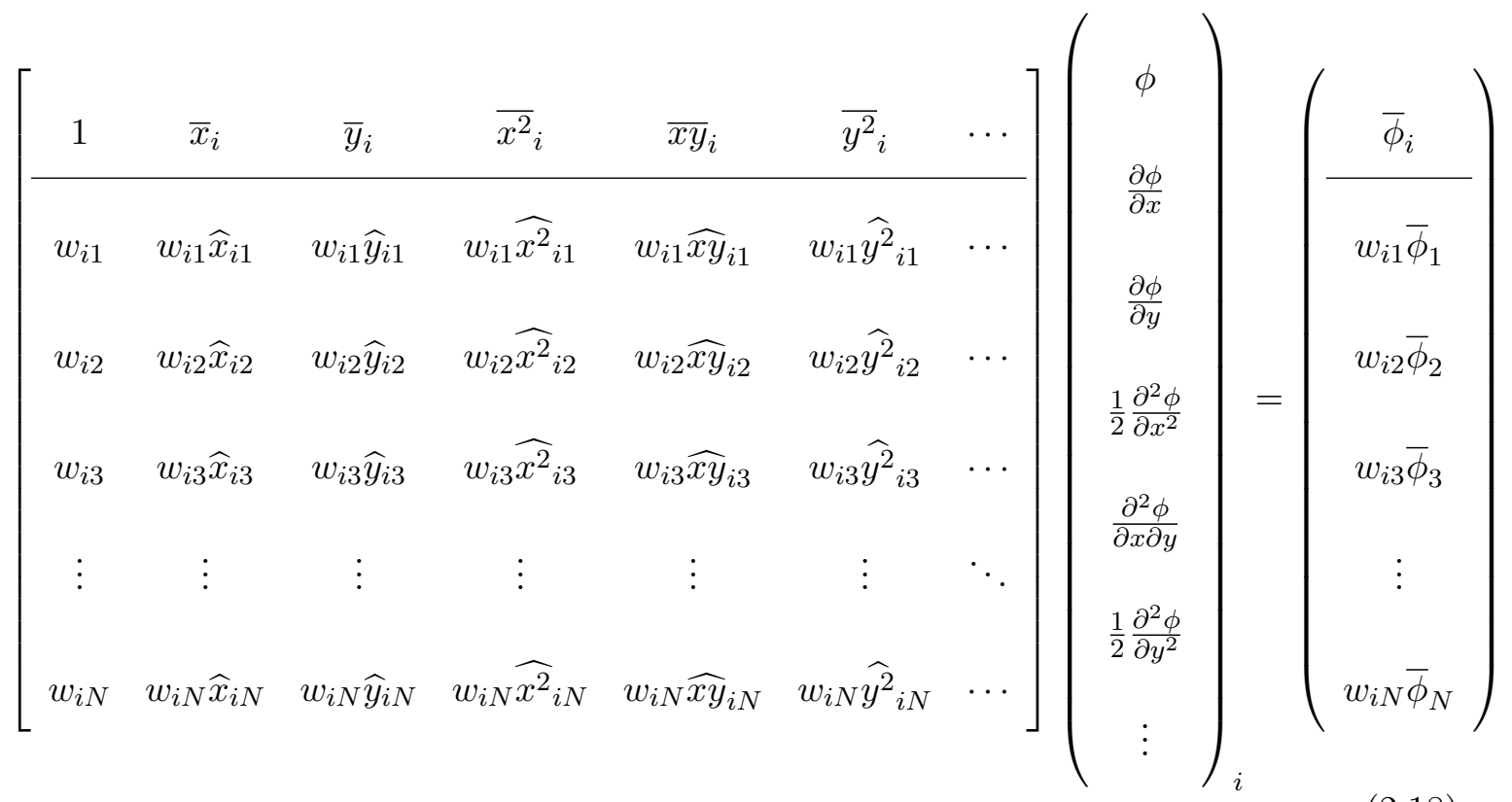

where the very first line is the mean constraint of the given control volume and the weights $w_{i j}$ are used to emphasize the effect of geometrically nearby control volumes. These weights are defined as

$$
w_{i j}=\frac{1}{\left|\vec{r}_{j}-\vec{r}_{i}\right|^{n}}
$$

where typically $n \in[0,2]$ and $\vec{r}$ is the position vector of the control-volume reference point. The choice of weighting scheme has been known to affect the conditioning and stability of the system $[30,29]$. The inverse-distance weighting $(n=1)$ is used as a default for most inviscid cases $[30,37]$.

In ANSLib, our unstructured flow-solver, the mean constraint is eliminated by Gauss elimination resulting in an unconstrained least-squares system which is then solved for 
every control volume using the Singular-Value-Decomposition (SVD) method. Moreover if we observe the least-squares system that is formed for every control volume, we notice that the leading matrix $\mathbf{A}$ on the left-hand side is purely geometrical and mesh dependent. Thus we can easily speed up the reconstruction process by precomputing and storing the pseudoinverse of this matrix. It can then be multiplied with the modified control-volume averages, the column matrix $\beta$, at each time step to obtain the new reconstruction coefficients.

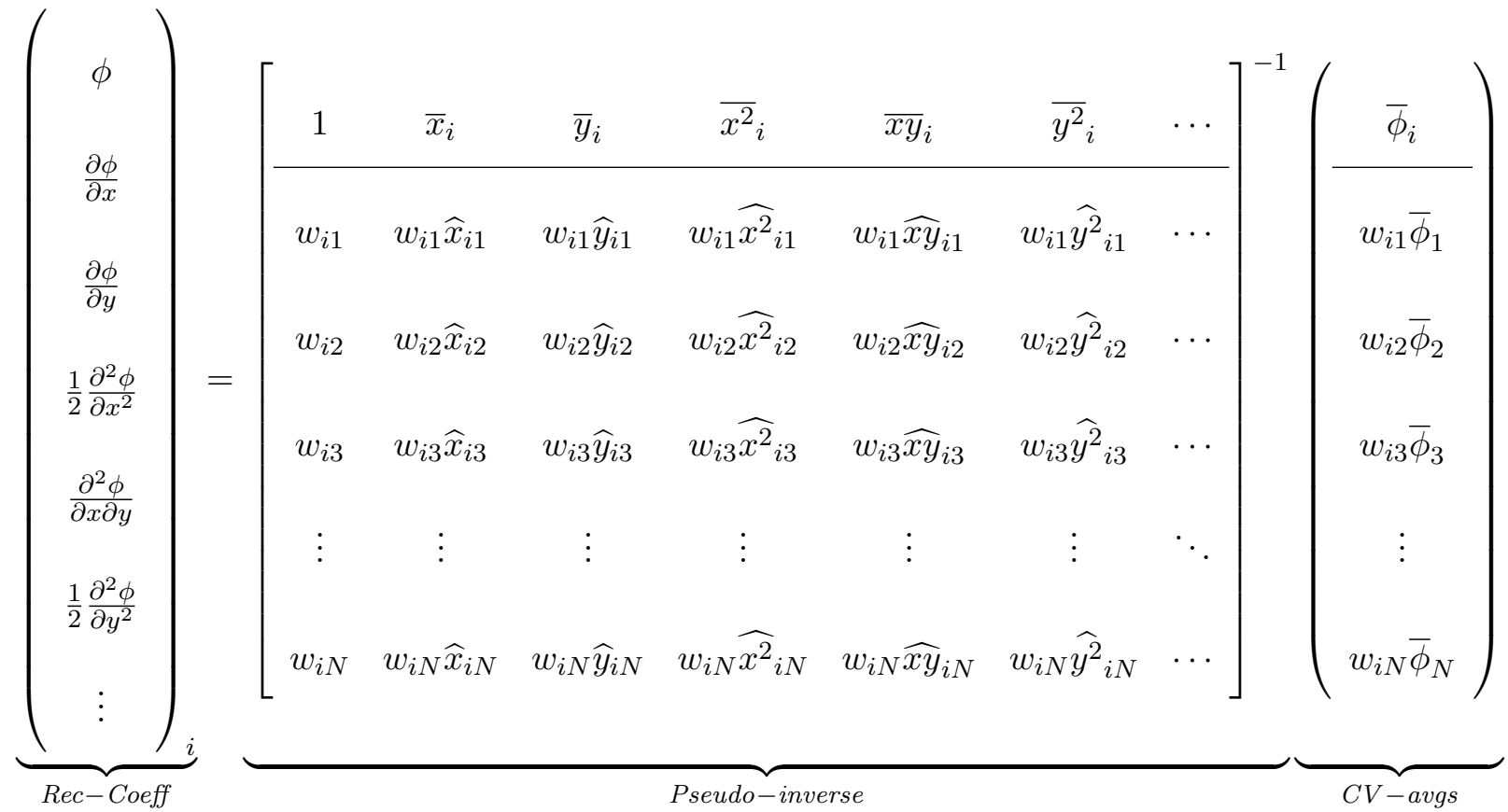

We will represent the pseudo-inverse in the following general form where the columns correspond to the neighbours and the rows correspond to the reconstruction coefficients:

$$
\mathcal{P} \operatorname{Inv}_{L S}=\left[\begin{array}{ccccc}
A_{11} & A_{12} & A_{13} & \cdots & A_{1 N} \\
A_{21} & A_{22} & A_{23} & \cdots & A_{2 N} \\
& & & & \\
A_{31} & A_{32} & A_{33} & \cdots & A_{3 N} \\
\vdots & \vdots & \vdots & \ddots & \vdots
\end{array}\right]
$$

Another very crucial aspect that should be addressed before any computational scheme is complete are its boundary treatment methods. The research presented here does not deal 
with the boundary control volumes and hence prior knowledge of the same is not essential to its understanding. A curious reader is directed to a detailed assessment of the same by Puneria $[47,46]$.

\subsubsection{Flux Evaluation}

As depicted by Equation 2.2, to find the rate of change of the unknown variable we need to evaluate the flux integral that tells us the net flux that crosses the boundaries of the given control volume. To do so we need the value of the numerical flux at the Gauss points on each edge of the cell so that a Gauss-quadrature integration can be performed using them. Thus, an accurate flux evaluation plays a vital role in the finite-volume computational scheme.

The evaluation of the numerical flux greatly depends on the physics of the problem being solved and the corresponding discretization scheme being used. There are broadly two main categories of fluxes. Convective fluxes describe the direct transport of some physical quantity across the control volume boundary and depend only on the solution. Diffusive fluxes describe processes such as mixing and heat conduction and depend on both the solution and its gradient. This research focus being the Poisson diffusion physics over a cell-centred finite-volume approach, we will in this section only discuss flux evaluation strategies for the same. A detailed explanation of convective fluxes can be found in the work of Jalali [19].

When using structured meshes, it is common practice to directly use a central differencing scheme on the cell averages from neighbouring control volumes to obtain a face gradient. Due to the inherent smoothness of a structured mesh, doing so rewards them with error cancellations that prevent any loss of accuracy. Unfortunately the same does not hold true for unstructured mesh solvers. The centroids which are used as cell reference points for unstructured cell-centred meshes are inconveniently not located on the perpendicular bisectors of the shared edge. This setback causes much harm to the accuracy of the face gradient and eventually reflects in the overall accuracy of the solver. This very concern has been addressed in the current research and a resolution will be presented in Chapter 3 with results in subsequent chapters. For now, we will discuss the current practices in unstructured mesh diffusive flux evaluation. 


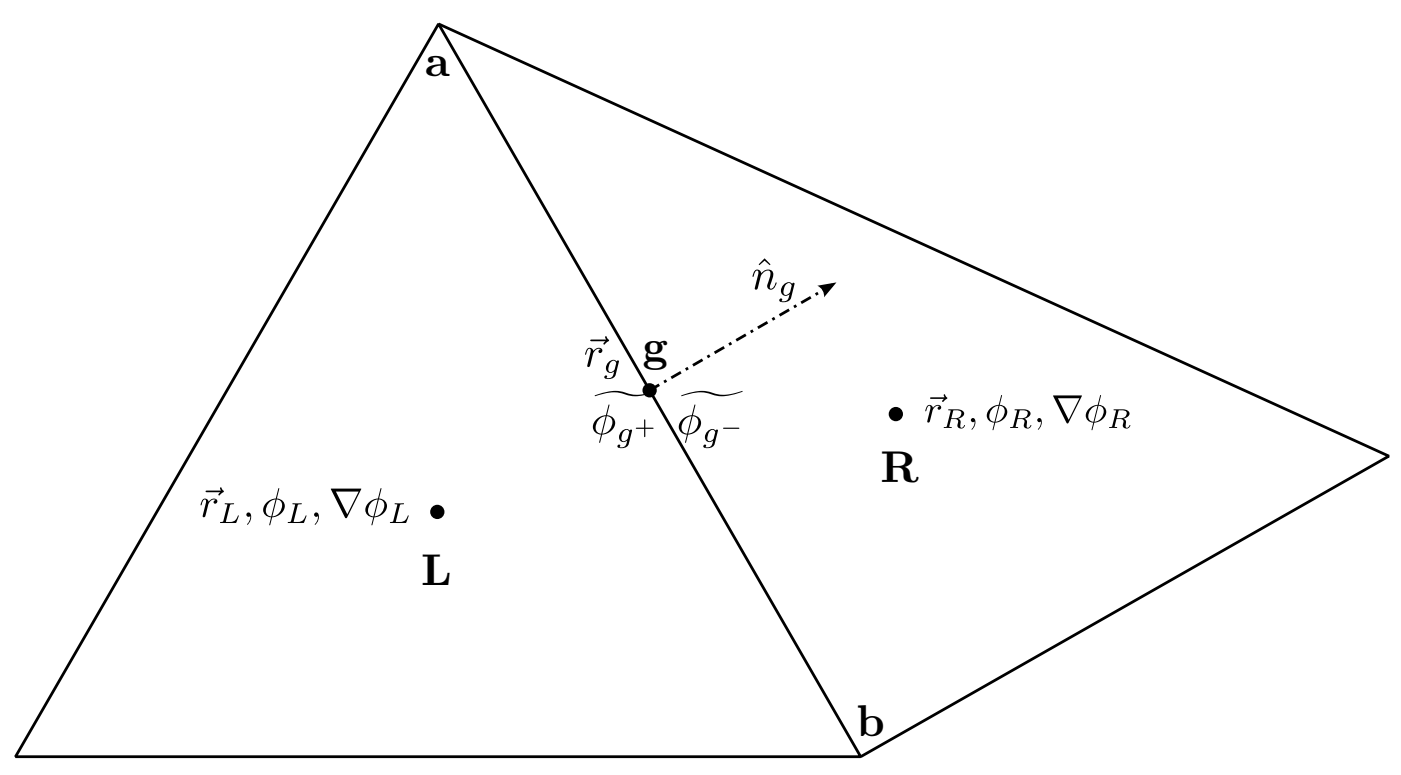

Figure 2.3: Mesh geometrical features

\subsubsection{Diffusive Flux Evaluation}

The diffusive flux for a face is given by

$$
\mathbb{F}_{D}=\int_{a b}(\nabla \phi)_{g} \cdot \hat{n} d s
$$

Thus in the case of diffusive fluxes, a unique value of gradients needs to be evaluated at all the face Gauss points. The easiest approach would be to take advantage of the gradient coefficients obtained from the reconstruction process explained in the previous section. Thus the gradients at the Gauss points, approximated using a Taylor series expansion from say the left control volume can be written as

$$
\widetilde{\nabla \phi_{g^{+}}}=\left(\begin{array}{c}
\left.\frac{\partial \phi}{\partial x}\right|_{L}+\left.\frac{\partial^{2} \phi}{\partial x^{2}}\right|_{L}\left(x_{g}-x_{L}\right)+\left.\frac{\partial^{2} \phi}{\partial x \partial y}\right|_{L}\left(y_{g}-y_{L}\right)+\cdots \\
\left.\frac{\partial \phi}{\partial y}\right|_{L}+\left.\frac{\partial^{2} \phi}{\partial y^{2}}\right|_{L}\left(y_{g}-y_{L}\right)+\left.\frac{\partial^{2} \phi}{\partial x \partial y}\right|_{L}\left(x_{g}-x_{L}\right)+\cdots
\end{array}\right)
$$

Figure 2.3 illustrates the various geometric terms utilized in this equation. A similar expression can be written from the perspective of the right control volume. But it should 
be noted that we require a unique value since the conservation property of the finite-volume method tells us that, across a shared edge, the out-flux from one cell should be equal to the in-flux into the neighbouring cell, that is,

$$
\int_{a b}(\nabla \phi)_{g^{+}} \cdot \hat{n} d s=\int_{a b}(\nabla \phi)_{g^{-}} \cdot \hat{n} d s
$$

This may seem straightforward, since we do have gradients at the cell reference points from the reconstruction and we just have to extrapolate the same using the Taylor series expansion, but there is a catch. The two gradient values at the shared Gauss point obtained from the reconstruction in the left and right cells are bound to be different. There is a discontinuity that arises due to the inherent reconstruction errors and non-smooth data in the reconstruction. Jalali et al. [21] have performed a comparative assessment of different approaches to resolve the same, including various averaging methods as well as addition of finite-difference term and jump terms for the calculation of face gradients. His findings have been presented in the following sections.

\subsubsection{Face Gradients}

The easiest way to resolve this discontinuity across the cell face would be an arithmetic averaging of the two gradient evaluations from the left and the right cell:

$$
\nabla \phi_{g}=\frac{1}{2}\left(\widetilde{\nabla \phi_{g^{+}}}+\widetilde{\nabla \phi_{g^{-}}}\right)
$$

Other variations incorporate some geometrical weighted averaging scheme such as linear interpolation or volume weighting. Linear interpolation works similar to a regular interpolation by taking into consideration the distance of the opposing cell reference points from the shared Gauss point, that is,

$$
\nabla \phi_{g}=\frac{1}{\left|\vec{r}_{L G}\right|+\left|\vec{r}_{R G}\right|}\left(\left|\vec{r}_{R G}\right| \widetilde{\nabla \phi_{g^{+}}}+\left|\vec{r}_{L G}\right| \widetilde{\nabla \phi_{g^{-}}}\right)
$$


while the volume weighted averaging emphasizes each gradients with their corresponding volumes (or areas in case of two-dimensions).

$$
\nabla \phi_{g}=\frac{1}{A_{L}+A_{R}}\left(A_{L} \widetilde{\nabla \phi_{g^{+}}}+A_{R} \widetilde{\nabla \phi_{g^{-}}}\right)
$$

Unfortunately using just the averaged value for the flux is insufficient for a robust discretization scheme making the scheme susceptible to high-frequency errors [21]. The use of finite-difference terms and jump terms have been proposed in the literature to mitigate this effect by introducing some damping into the system. Among these the use of the jump term has been shown to be most effective.

\subsubsection{Jump Terms}

The jump term was first developed by Nishikawa [35] and was shown to be a very effective damping agent for high-frequency errors. It accounts for the discontinuity in the solution across the control volume interface and is added directly to the flux in the face normal direction using the following formulation

$$
(\nabla \phi)_{j u m p}=\frac{\alpha}{\left|\vec{r}_{L R} \cdot \hat{n}_{g}\right|}\left(\widetilde{\phi_{g^{+}}}-\widetilde{\phi_{g^{-}}}\right)
$$

where $\alpha$ is a constant known as the jump term coefficient, $\vec{r}_{L R}$ is the distance vector joining the left and right cell reference points, $\hat{n}_{g}$ is the face normal unit-vector at the Gauss-point $g$ and $\widetilde{\phi_{g^{+}}}, \widetilde{\phi_{g^{-}}}$are the solution values at the Gauss-point obtained using Taylor series expansion from the left and right control volumes respectively. The jump term coefficient greatly affects the efficacy of the jump term. Jalali [21] suggests a value of $4 / 3$ as optimal for the jump coefficient. Jump terms with optimal jump coefficients have been demonstrated to considerably increase the solution accuracy in the case of diffusion schemes $[21,60]$. As will be later discussed our implementation of the jump term improves upon this current design. Our findings indicate a significant improvement in the accuracy as well as stability of our scheme upon the addition of a jump term.

The various flux evaluation strategies have been summarized in Table 2.1 with Figure 2.3 


\begin{tabular}{cc}
\hline Strategy & Formula \\
\hline \hline Arithmetic averaging & $\frac{1}{2}\left(\widetilde{\nabla \phi_{g^{+}}}+\widetilde{\nabla \phi_{g^{-}}}\right)$ \\
\hline Linear interpolation & $\frac{1}{\vec{r}_{L g}|+| \vec{r}_{R g} \mid}\left(\left|\vec{r}_{R g}\right| \widetilde{\nabla \phi_{g^{+}}}+\left|\vec{r}_{L g}\right| \widetilde{\nabla \phi_{g^{-}}}\right)$ \\
\hline Volume-weighted & $\frac{1}{A_{L}+A_{R}}\left(A_{L} \widetilde{\nabla \phi_{g^{+}}}+A_{R} \widetilde{\nabla \phi_{g^{-}}}\right)$ \\
\hline \hline Jump Term & $\frac{\alpha}{\left.\left|\vec{r}_{L R^{\prime} \cdot \hat{n}_{g}}\right| \widetilde{\phi_{g^{+}}}-\widetilde{\phi_{g^{-}}}\right)}$ \\
\hline
\end{tabular}

Table 2.1: Flux Evaluation Strategies

illustrating the individual geometric terms.

\subsubsection{Flux Integration}

A Gauss quadrature integration rule is a common choice for numerical integration since it requires the integrands to be evaluated at only a few specific points known as Gauss quadrature points. The greater the accuracy required, the greater is the number of Gauss points chosen per edge. For instance, one Gauss point located at the edge-mid-side would suffice for first and second-order accuracy, while third and fourth order accurate integration would demand two Gauss points per edge of the cell. The exact location of the Gauss points for up to 4 th order is given by Table 2.2. Figure 2.4 illustrates the same schematically. The accuracy of the flux integration has to be set equal to or higher than the solution reconstruction accuracy lest all the reconstruction accuracy achieved be lost trivially.

Once we have the numerical flux values at the Gauss points from the previous stage, a flux integration can be performed over that edge of the control volume using this Gauss quadrature rule. The total flux integral in the Equation 2.3 for the control volume is then

\begin{tabular}{cc}
\hline Order of Flux Integration & Location (in terms of edge vertices) \\
\hline \hline Second-order & $\vec{r}_{g}=\left(\frac{\vec{r}_{B}+\vec{r}_{A}}{2}\right)$ \\
\hline Third and Fourth order & $\vec{r}_{g}=\left(\frac{\vec{r}_{B}+\vec{r}_{A}}{2}\right) \pm\left(\frac{\vec{r}_{B}-\vec{r}_{A}}{2 \sqrt{3}}\right)$ \\
\hline
\end{tabular}

Table 2.2: Gauss-point locations 


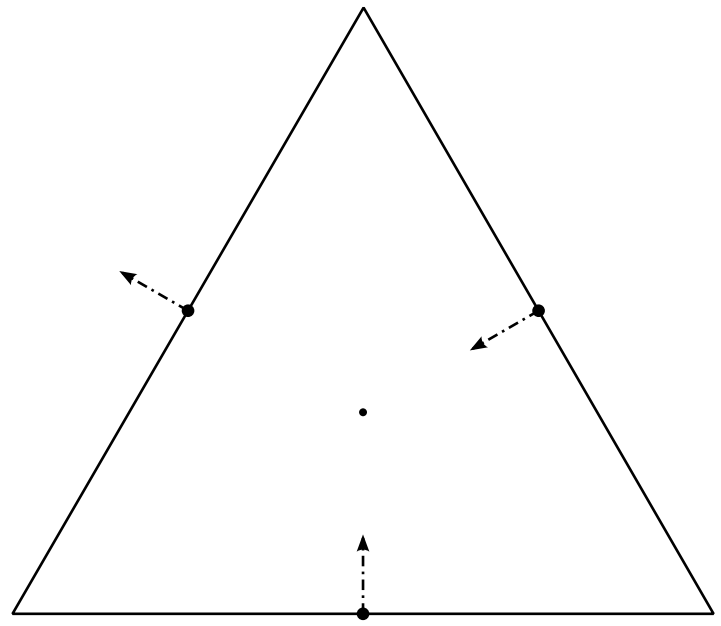

(a) Second-order (1 Gauss-point per edge)

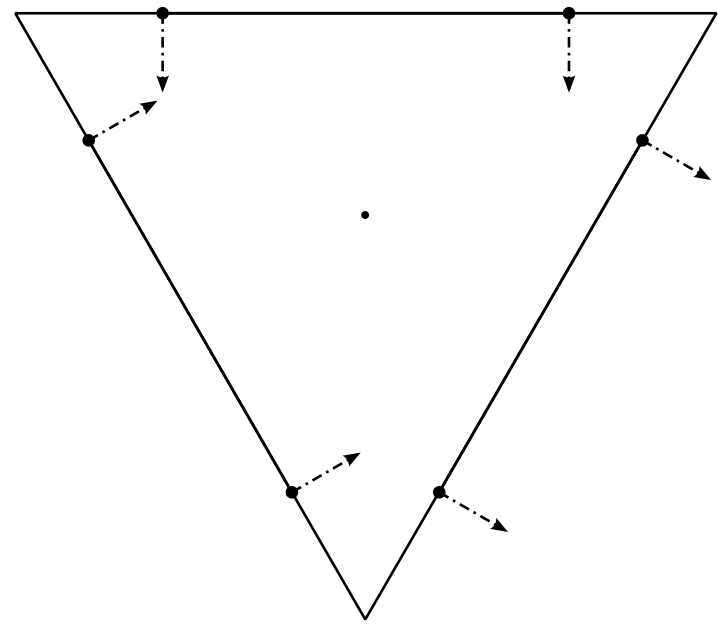

(b) Third or fourth order (2 Gauss-point per edge)

Figure 2.4: Gauss quadrature points schematic

simply a summation of the individual integrals along each edge of the cell. A more indepth analysis and explanation of the flux integration process can be found in the works of Ollivier-Gooch and Van Altena [43] and Van Altena [55].

\subsubsection{Time Evolution}

In the present work we are concerned with the solution to the Poisson equation which is a steady-state model equation. In such cases the time-derivative in Equation 2.3 is made redundant and thus may be eliminated. The task then is to find the solution to the system of equations defining the discrete flux integrals, which can be solved by any matrix solution technique to obtain the steady-state Poisson solution. How well and how quickly we are able achieve that steady-state solution is described by the term, convergence of solution.

Although this is essentially straight forward it greatly lacks the robustness that is often demanded from all flow-solvers. To circumvent this drawback ANSLib implements a technique known as Pseudo-Timestepping wherein we take (implicit) time steps towards the steady state solution instead of solving for it at one go. This imparts the desired robustness in the steady-state time evolution without sacrificing solution accuracy. Hence in our research we use this Pseudo-Timestepping technique. Moreover, by doing we we can hope to apply similar accuracy results we see in the model cases even in the future applications 
of Euler and Navier-Stokes equations which are not necessarily steady state problems.

This and other time evolution algorithms that are used within ANSLib have been very well documented by Nejat [33] and Michalak [30, 31].

\section{$2.2 \quad$ Flux Error Reduction}

Few other researchers have attempted to address similar motivations as those described in Section 1.1.

Katz and Sankaran [22] proposed a novel approach to obtain third-order accurate solution to inviscid problems by means of a flux correction term. Unlike our motivation, their ultimate goal was to obtain higher accuracy for the solution rather than the gradients. Their flux correction term is aimed at specifically improving the truncation error through error-cancellations which they show translates to improvements in the accuracy of the solution. It is also unique since it can be directly added to any existing second-order accurate vertex-centred (also known as node-centred) scheme. However, as they mention, this flux correction term is unfortunately limited in its applications to vertex-centred linear Galerkin schemes. Moreover, the computation of this term is itself not trivial and requires at least a second-order accurate estimate of the gradient of the flux. This flux gradient is computed using a quadratic least squares approximation. The additional computational time required per iteration for this corrected scheme was reported to be around $50 \%$ more than the regular vertex-centred linear Galerkin schemes.

This flux correction scheme was also extended to unsteady viscous flows by Pincock and Katz [44]. As can be anticipated, the correction terms required for the viscous fluxes are significantly more computationally intensive, with a 40-70\% increase over a second-order finite-volume method. Unfortunately, comparisons to a third-order finite-volume method were not shown, which makes it tough to compare our schemes. Here, for the viscous terms, they propose a new cubic polynomial "Jacobian-averaged" finite-element mapping to compute the gradients within each control-volume, which contributes to the significant costs. They report some instabilities with regards to certain problems and also express their work in progress to enable the use of larger time steps. 
Another equally interesting approach was developed by Nishikawa for the diffusion equation [34] and later extended to encompass advection as well [36]. The key concept he proposes is the use of a first-order hyperbolic system that is equivalent to the the regular second-order diffusion equation, albeit only in the steady state. A secondary yet remarkable feature is that his new scheme gives second-order accuracy for both the solution as well as the gradients. Although developed for a second-order residual-distribution scheme, he suggests that it could be developed in a similar manner for finite-difference and finitevolume schemes as well. At its current state of development it only works with an explicit time advancing strategy and an implicit time advance would ruin the solution accuracy. However, they do report an advantage of being able to use much larger explicit time steps than other regular Galerkin schemes. 


\section{Chapter 3}

\section{$\mathbf{H}_{1}$ Finite-Volume Scheme}

In this chapter we present the theory behind our proposed $\mathrm{H}_{1}$ finite-volume scheme. We will describe our scheme in one dimension before examining the two-dimensional scheme in detail.

Our overall objective can be broadly put forth as striking a balance between the best features of third-order accurate least-squares with nearly the speed and simplicity of secondorder methods. An overview of the scheme that we propose is as follows

1. Compute a reconstruction that is second order for both the solution and the gradients.

2. Utilize this reconstruction to evaluate a second-order diffusive flux at the Gauss points.

3. Use single point Gauss-quadrature (located on the face mid-side) to obtain the flux integral for each control volume.

4. Update the solution states in every control volume.

The first step is in essence to obtain the gradients to second-order accuracy at the controlvolume reference points with the least computational effort while the second step is a new flux discretization we propose. Our modifications do not apply to the last two steps, the regular flux-integral evaluation and time-stepping strategies, which were discussed in brief in section 2.1 .

\section{$3.1 \quad \mathrm{H}_{1}$ Reconstruction}

The first step in our new scheme is to obtain the gradients to second-order accuracy at the control volume reference points. A regular second-order reconstruction scheme computes just the solution and its first-derivative (gradient) coefficients and as such requires just three 
face neighbours from the first layer in Figure 2.2 to complete its reconstruction stencil. It thus boasts a relatively cheap pseudo-inverse that has the general form of

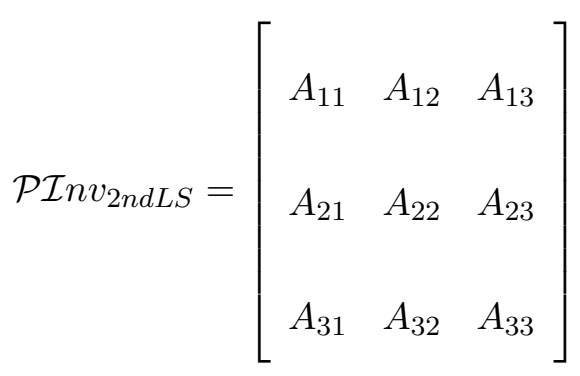

In contrast a third-order reconstruction typically uses nine neighbours to enable the estimation of the three additional second-derivative (quadratic) coefficients. Hence its leastsquares system and pseudo-inverse are more complex and computationally intensive. This results in a third-order accurate approximation to the solution and a second-order accurate approximation to the solution gradient.

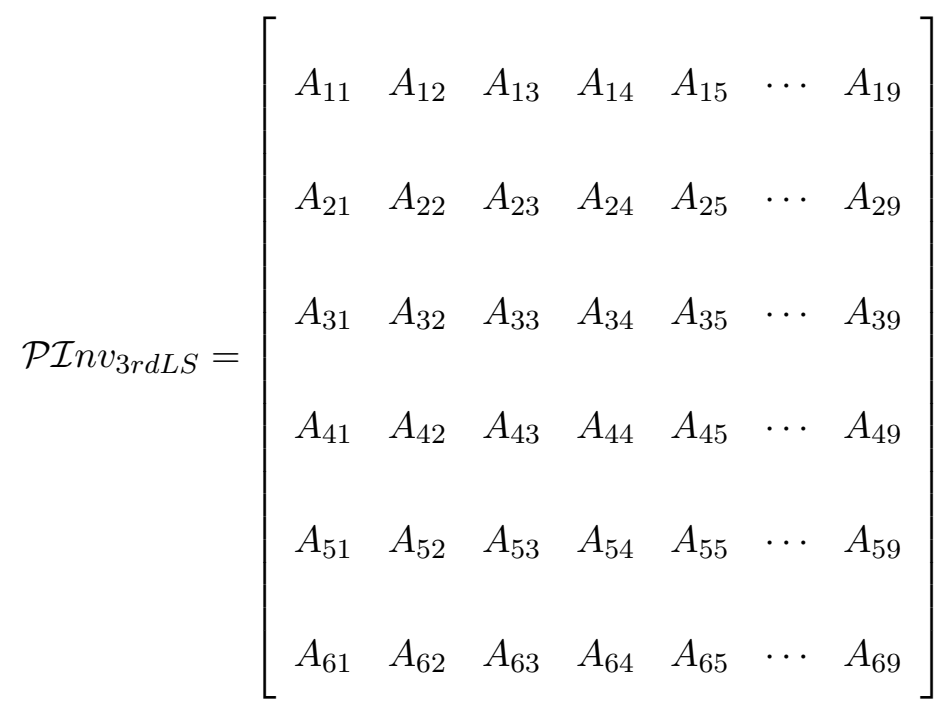

With $\mathrm{H}_{1}$ reconstruction we do not need to compute the three additional second-derivative coefficients as will be evidenced in the next subsection. Thus this enables us to eliminate the last three rows corresponding to the quadratic coefficients. This saves us considerable resources and computational time when repeatedly performing the matrix-vector multiplication between the pseudo-inverse and the control-volume averages to obtain the solution reconstruction coefficients at each time step. It also provides a substantial gain in efficiency 


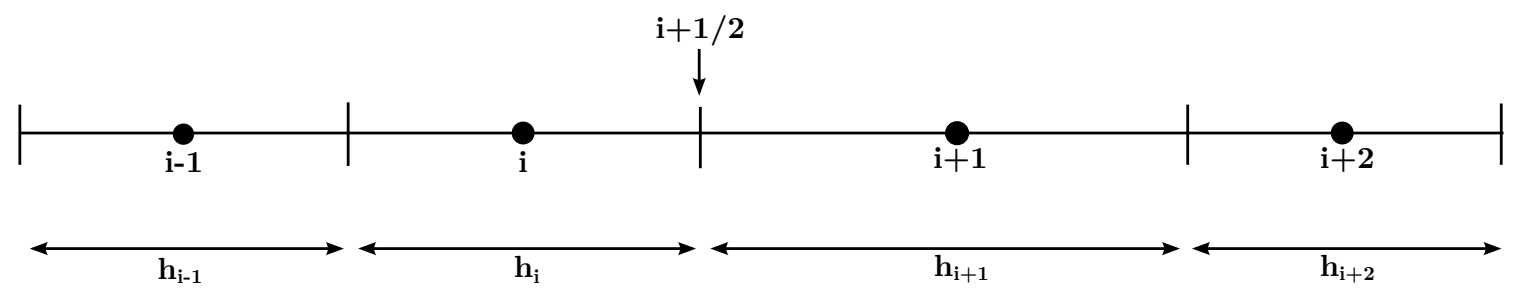

Figure 3.1: 1D Non-uniform Grid Stencil

since we get a relatively large jump in accuracy without the laborious computation that the quadratic terms would demand. Note that the three pseudo-inverse rows that we are left with are exactly the same as those from a third-order reconstruction.

$$
\mathcal{P}{\mathcal{I} n v_{H_{1}}}=\left[\begin{array}{cccccccc}
A_{11} & A_{12} & A_{13} & A_{14} & A_{15} & \cdots & A_{19} \\
A_{21} & A_{22} & A_{23} & A_{24} & A_{25} & \cdots & A_{29} \\
A_{31} & A_{32} & A_{33} & A_{34} & A_{35} & \cdots & A_{39}
\end{array}\right]
$$

\section{$3.2 \quad \mathrm{H}_{1}$ Flux Discretization}

Once we have obtained the necessary second-order gradients at the control-volume reference points we must translate those to the Gauss points while maintaining our accuracy. Here we discuss our new approach to the flux discretization at the Gauss point which counters the loss of error cancellations due to the irregularity of the mesh.

\subsubsection{Theory in One Dimension}

The quickest way to test the waters with a proof of concept is to take a look at what can be accomplished on a one-dimensional grid. In our case that turns out to be relatively straightforward. We will expand on the same with reference to the one-dimensional non-uniform grid stencil shown in Figure 3.1; this is the one-dimensional analog to an unstructured mesh.

Consider the Taylor series expansion of the first derivative about the cell centres in terms 
of the derivative at the cell interface. Note that the reconstructed first derivatives $\left(\frac{\widetilde{d \phi}}{d x}\right)$ are a second-order approximation to the exact derivatives $\left(\frac{d \phi}{d x}\right)$ at the cell reference point.

$$
\begin{aligned}
& \left.\frac{\widetilde{d \phi}}{d x}\right|_{i}+O\left(h^{2}\right)=\left.\frac{d \phi}{d x}\right|_{i}=\left.\frac{d \phi}{d x}\right|_{i+1 / 2}-\left.\frac{h_{i}}{2} \frac{d^{2} \phi}{d x^{2}}\right|_{i+1 / 2}+O\left(h^{2}\right) \\
& \left.\frac{\widetilde{d \phi}}{d x}\right|_{i+1}+O\left(h^{2}\right)=\left.\frac{d \phi}{d x}\right|_{i+1}=\left.\frac{d \phi}{d x}\right|_{i+1 / 2}+\left.\frac{h_{i+1}}{2} \frac{d^{2} \phi}{d x^{2}}\right|_{i+1 / 2}+O\left(h^{2}\right)
\end{aligned}
$$

Now normally for a least-squares reconstruction we would take a direct average of the above two Equations 3.4 and 3.5, to obtain the derivative at the cell interface

$$
\left.\frac{d \phi}{d x}\right|_{i+1 / 2}=\frac{1}{2}\left\{\left.\frac{\widetilde{d \phi}}{d x}\right|_{i}+\left.\widetilde{d \phi}\right|_{i+1}\right\}-\left.\left(\frac{h_{i+1}-h_{i}}{4}\right) \frac{d^{2} \phi}{d x^{2}}\right|_{i+1 / 2}+O\left(h^{2}\right)
$$

For structured (uniform) meshes the second term on the right hand side of Equation 3.6 would cancel out upon taking an arithmetic average; unfortunately the same cannot be said of unstructured meshes. Thus structured meshes enjoy a second-order accurate derivative while unstructured mesh counterparts have to contend with first-order derivatives.

Instead of using a simple arithmetic average, if we were to eliminate each of the firstorder terms on the right hand side of Equations 3.4 and 3.5 with a linear interpolation, then we can expect a gain in accuracy even for unstructured (non-uniform) grids.

$$
\left.\frac{d \phi}{d x}\right|_{i+1 / 2}=\frac{1}{\left(h_{i+1}+h_{i}\right)}\left\{\left.h_{i+1} \cdot \frac{\widetilde{d \phi}}{d x}\right|_{i}+\left.h_{i} \cdot \frac{\widetilde{d \phi}}{d x}\right|_{i+1}\right\}+O\left(h^{2}\right)
$$

This enables us to gain back second-order accuracy even in unstructured meshes without the use of the higher derivatives. Thus the higher derivatives obtained from the reconstruction are made redundant and need not be computed. In the next subsection we look at extending this technique to two dimensions.

\subsubsection{Extension to Two Dimensions}

In two dimensions we essentially only require the component of the gradient in the normal direction to compute the diffusive flux at any given control-volume interface. The 


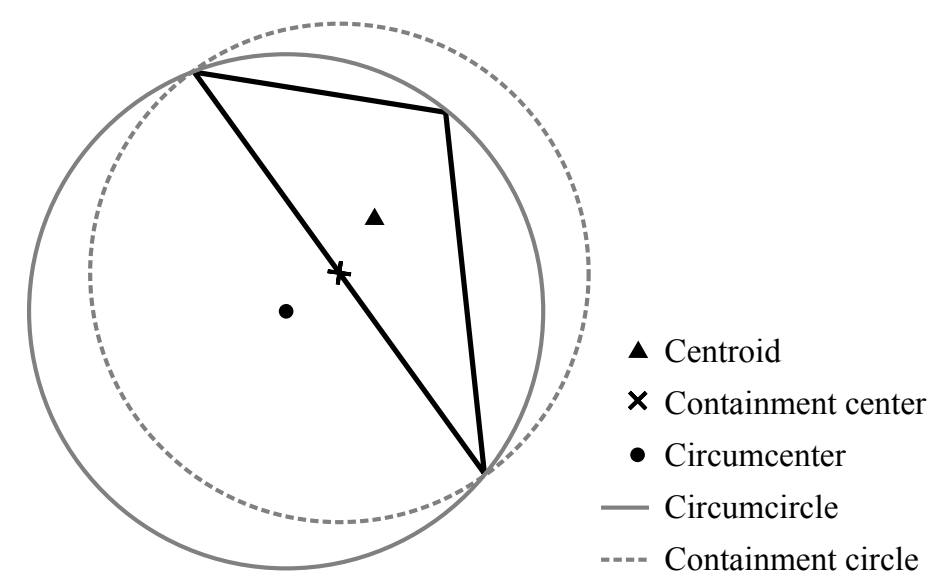

Figure 3.2: Various control-volume reference centres

traditional use of centroids as the control-volume reference point has proven to be a stable and robust choice. However, obtaining the second-order normal gradient components using centroids necessitates the use of the second derivatives. Another control-volume reference point candidate that has been rarely looked into is the circumcentre. A circumcentre is defined as the point where the perpendicular bisectors of the faces of a triangle intersect. Obtaining the second-order normal gradient components using them should be easier, since they always lie on the face normal through the edge mid-side. Unfortunately the use of circumcentres as control volume reference points leads to instabilities, which will be further discussed in section 6.1. The use of containment centres instead provides better stability without hampering the accuracy gains. Containment centres are defined as the centres of the smallest circle that can enclose a given triangle. The trio of control-volume reference points - namely the centroid, the circumcentre and the containment-centre - are illustrated in Figure 3.2. Our flux discretization strategy using a combinations of circumcentres or containment centres is presented below.

\subsubsection{Circumcentres}

Circumcentres are the prime candidate for control-volume reference points for this scheme, since they always lie on the perpendicular bisector of the faces. However with unstructured meshes the triangles are not guaranteed to be acute. Hence there will definitely arise scenarios where the circumcentre falls outside the cell or worse coincides with the circumcentre 


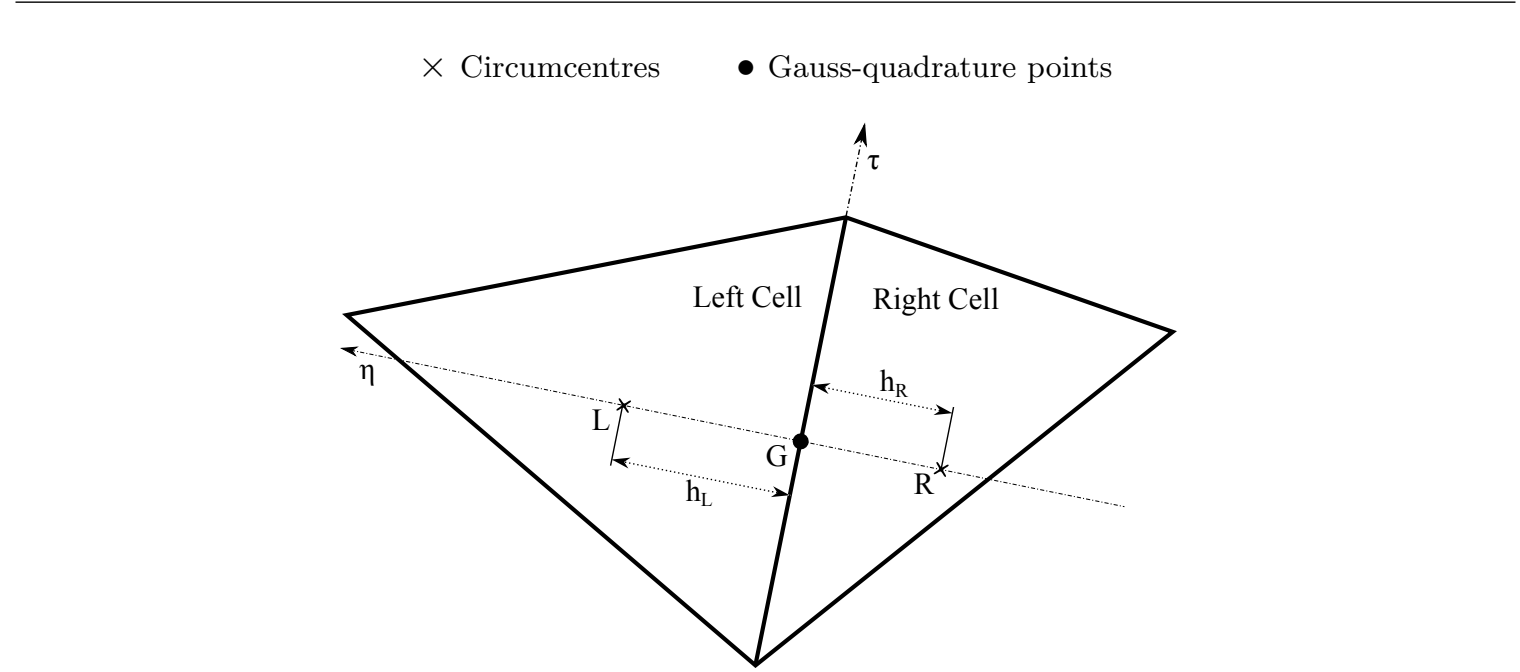

(a) The Good circumcentre case

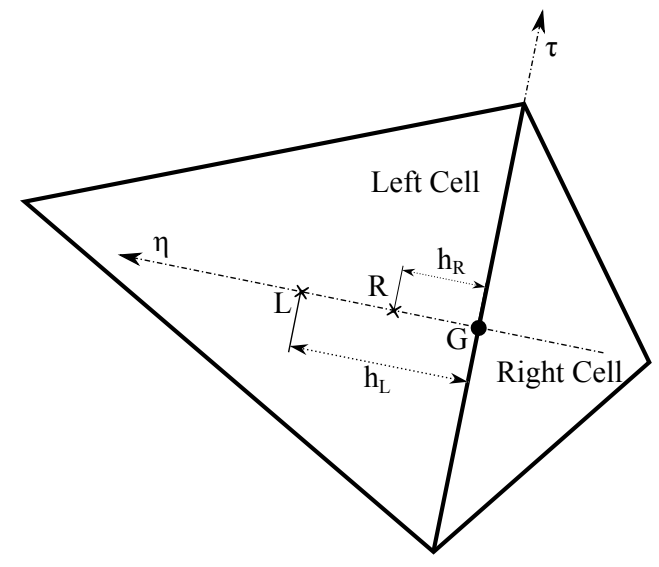

(b) The Bad circumcentre case

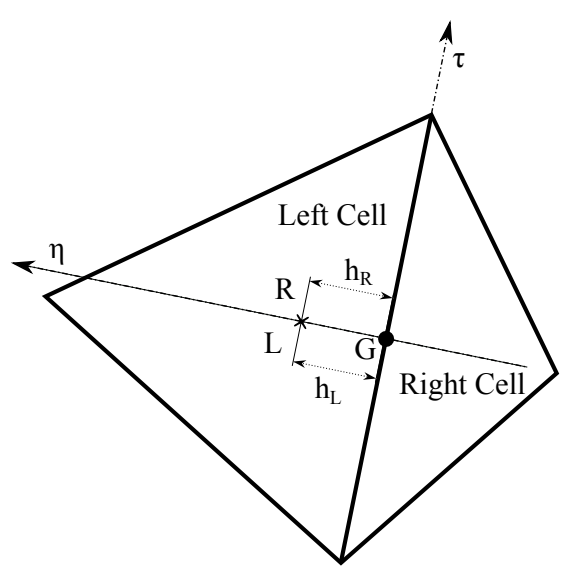

(c) The Ugly circumcentre case

Figure 3.3: Circumcentre cases

of a neighbour. These situations fall into the following three categories. Treating $h_{L}$ and $h_{R}$ as signed normal distance to the Gauss point, each positive into its own cell, enables the identification and handling of these scenarios.

The Good: This is the best case scenario - and the most common for isotropic meshes — where the cells on either side of the face are acute and hence the circumcentres are well behaved as illustrated by Figure 3.3a. The circumcentres lie on either side of the Gauss point, so we can directly implement a linear interpolation formula similar to that in one dimension given by Equation 3.7. We note that the same formula can also be used in cases where both the triangles are obtuse in which case the signed distances, being both negative, would just negate each other. The second-order gradient at the 
Gauss point is given by

$$
\left.\frac{\partial \phi}{\partial \eta}\right|_{g}=\frac{1}{\left(h_{L}+h_{R}\right)}\left\{\left.h_{R} \frac{\widetilde{\partial \phi}}{\partial \eta}\right|_{L}+\left.h_{L} \frac{\widetilde{\partial \phi}}{\partial \eta}\right|_{R}\right\}+O\left(h^{2}\right)
$$

The Bad: Here we face an obtuse triangle on one side of the face as illustrated by Figure 3.3b. Thus both the circumcentres are on the same side of the Gauss point and one of those signed distances is negative. We still obtain a second-order gradient at the Gauss point using Equation 3.8 thanks to the linear extrapolation formula formed implicitly by the use of signed distances.

The Ugly: This is the last and worst scenario, which occurs when the two circumcentres coincide. In such cases we revert to arithmetic averaging of cell gradients, as interpolation is impossible.

$$
\left.\frac{\partial \phi}{\partial \eta}\right|_{g}=\frac{1}{2}\left\{\left.\frac{\widetilde{\partial \phi}}{\partial \eta}\right|_{L}+\left.\frac{\widetilde{\partial \phi}}{\partial \eta}\right|_{R}\right\}+O(h)
$$

However, these cases were later identified as the root cause of- instabilities to be explained in Section 6.1, and hence were eliminated with the use of containment centres.

\subsubsection{Containment Centres}

Containment centres are defined as the centres of the smallest circle that can enclose a given triangle. They are identical to the circumcentre of an acute triangle but have the added advantage of not spilling out of the triangle when it is obtuse. In that scenario the containment centre is the mid-side of the longest edge of the triangle, which enables us to directly utilize its value for our face gradient. This should theoretically be ideal for that specific Gauss point but hurt accuracy for the flux at the remaining Gauss points on the other faces.

$$
\left.\frac{\partial \phi}{\partial \eta}\right|_{g}=\left\{\left.\frac{\widetilde{\partial \phi}}{\partial \eta}\right|_{R}\right\}+O\left(h^{2}\right)
$$

Two approaches to the implementation of containment centres can be thought of. First 


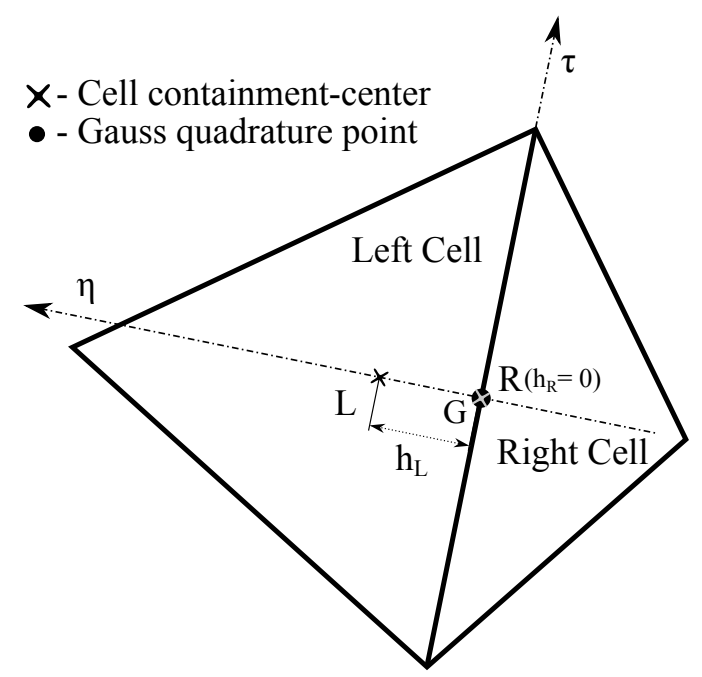

Figure 3.4: The obtuse containment centre

would be a blanket implementation where containment centres are implemented throughout the mesh thus eliminating the bad and ugly cases. The other approach would use containment centres to counter the ugly circumcentre cases, which were the root cause of the stability problem. The latter proved much more accurate and hence results presented in this thesis use this approach.

\subsubsection{High-Accuracy Jump Term}

As discussed in subsection 2.1.3.3, the jump term that accounts for the difference in the reconstructed solution in the two cells at the Gauss point plays a significant role in enhancing the stability of a diffusion scheme and making it more robust. Hence we wished to take advantage of its damping effects. However a regular jump term would adversely affect the accuracy of our discretization since it would only be first-order accurate, given that the solution is second-order accurate. Hence we have formulated a new jump term with higher-order accuracy that would enhance stability while serving our accuracy needs.

The first requirement to obtain the high-accuracy jump term is an estimate of the second-derivative at the Gauss point in the normal direction. We obtain this estimate using a central-difference formulation between the second-order gradients at the control-volume 
reference points.

$$
\left.\left.\frac{\partial^{2} \phi}{\partial \eta^{2}}\right|_{L} \approx \frac{\partial^{2} \phi}{\partial \eta^{2}}\right|_{m i d}=\frac{\left\{\left.\frac{\widetilde{\partial \phi}}{\partial \eta}\right|_{L}-\left.\frac{\widetilde{\partial \phi}}{\partial \eta}\right|_{R}\right\}}{h_{L R}+\epsilon}+O\left(h^{2}\right)
$$

We then use this to estimate more accurate left and right solution states at the Gauss point of the face.

$$
\widetilde{\phi_{g^{+}}} \approx\left(\widetilde{\phi_{L}}+\left.\frac{\partial \phi}{\partial x}\right|_{L} h_{L x}+\left.\frac{\partial \phi}{\partial y}\right|_{L} h_{L y}\right)+\left.\frac{\partial^{2} \phi}{\partial \eta^{2}}\right|_{L} \frac{h_{L}^{2}}{2}
$$

where the term in brackets on the right-hand side is evaluated implicitly from the gradient reconstruction.

We can then use these solution states in the jump term formulation to enhance its accuracy.

$$
(\nabla \phi)_{j u m p}=\frac{\alpha}{h_{\text {EdgeLength }}}\left(\widetilde{\phi_{g^{+}}}-\widetilde{\phi_{g^{-}}}\right)
$$

The constant $\epsilon$, with a value of $10^{-9}$, as well as the face edge-length being used as the length base, are precautions in place to avoid scenarios where the circumcentres or containment centres coincide and we would be dividing by zero. 


\section{Chapter 4}

\section{Analytical Tests and Analysis}

As we discussed in the previous chapter, a p-order-accurate solution reconstruction will produce (p-1)-order-accurate gradients which in turn leads to (p-1)-order diffusive flux evaluation. Ollivier-Gooch and Van Altena [43] were able to explain this loss in accuracy by analytically solving the least-squares system on uniform triangular meshes. They analyzed the flux integral, and its associated truncation error, for the Laplacian operator obtained using linear (second-order), quadratic (third-order) and cubic (fourth-order) reconstructions. The Laplace equation being a simplified form of the Poisson equation without a source term, can be formulated as:

$$
\nabla^{2} \phi=0
$$

Given the uniform and symmetric nature of the mesh, computations become simplified enough to be handled by symbolic mathematical software tools such as Maple, but are still too laborious to be done by hand.

Here we extend the analysis presented by [43], to our proposed $\mathrm{H}_{1}$ reconstruction scheme and compare it with the second and third-order least-square schemes.

\subsection{Methodology}

To begin, a coordinate and labelling system is created for a uniform, equilateral triangular mesh as illustrated by Figure 4.1. Each cell-centred control-volume triangle has edges of length $\mathrm{h}$. The index coordinates $(i, j)$, used for identifying the cells, do not run parallel to the Cartesian coordinates $(x, y)$, with the $\mathrm{j}$ axis running from the bottom left to top right corner. Each parallelogram with sides parallel to the i and $\mathrm{j}$ axes is labelled with a unique $(i, j)$ pair and the respective triangles are distinguished as being either up $(\Delta)$ or down $(\nabla)$ 


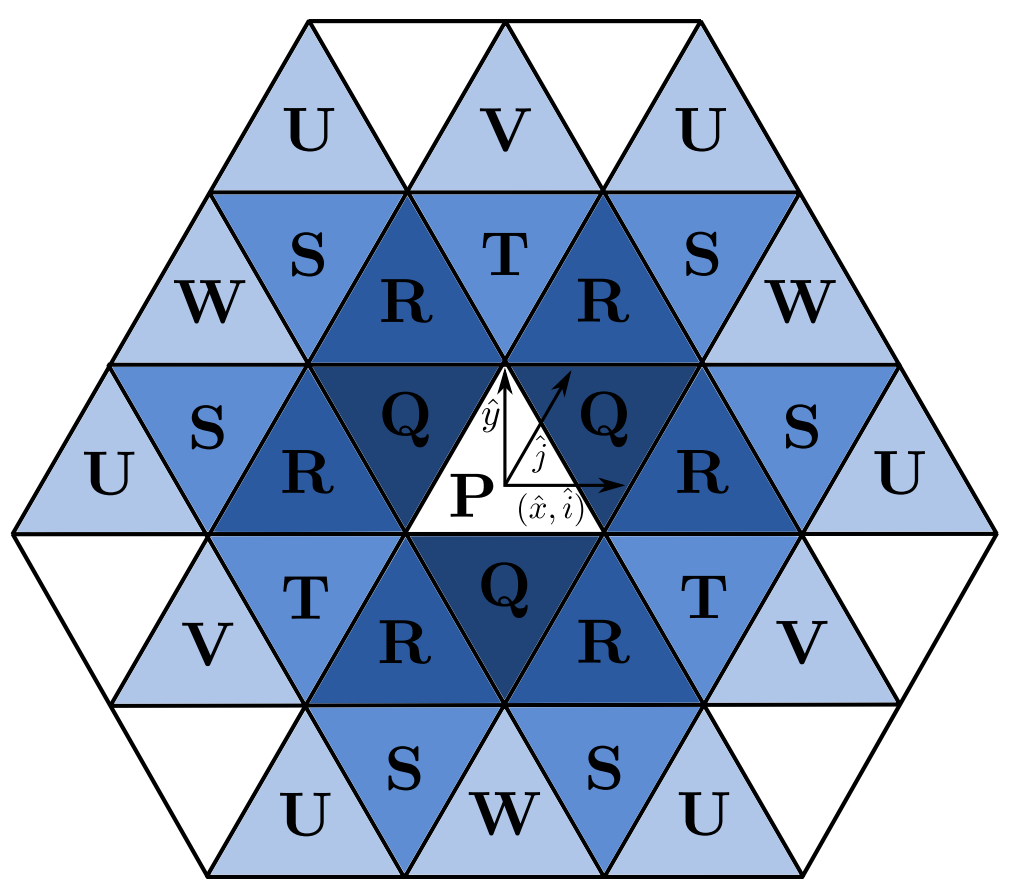

Figure 4.1: Analytical Mesh coordinates and cell labels

pointing. Triangles have also been classified to belong to a particular family based on their coefficients for the flux integral. The control volumes from the same family share the same coefficients due to the symmetry of the mesh. We will perform our reconstruction about the central $P$ cell.

The control volume moments are computed by analytical integration. We can then prepare the finite-volume framework that was described in chapter 2 to analytically solve for the solution, diffusive flux and the flux integral. The next section details our results and analysis.

\subsection{Analytical Results}

The results of our analysis have been summarized in Table 4.1. Further discussion on each of the cases follow.

\subsubsection{Linear Reconstruction}

Beginning with the linear (second-order) reconstruction, as already discussed the first three neighbours (Q's) are sufficient to satisfy the least-squares constraint requirements. All 


\begin{tabular}{|c|c|c|c|c|}
\hline & \multicolumn{4}{|c|}{ Reconstruction type } \\
\hline & Linear & Regular $\mathrm{H}_{1}$ & Extended $\mathrm{H}_{1}$ & Quadratic \\
\hline $\begin{array}{l}\text { Recon. stencil } \\
\text { (weight) }\end{array}$ & $\mathrm{Q}\left(3 / h^{2}\right)$ & $\begin{array}{l}\mathrm{Q}\left(3 / h^{2}\right) \\
\mathrm{R}\left(1 / h^{2}\right)\end{array}$ & $\begin{array}{l}\mathrm{Q}\left(3 / h^{2}\right), \\
\mathrm{R}\left(1 / h^{2}\right), \\
\mathrm{T}\left(3 / 4 h^{2}\right)\end{array}$ & $\begin{array}{l}\mathrm{Q}\left(3 / h^{2}\right) \\
\mathrm{R}\left(1 / h^{2}\right)\end{array}$ \\
\hline Leading T.E. term & $\frac{h^{2}}{16} \nabla^{2} \nabla^{2} \phi$ & $\begin{aligned} & \frac{h \sqrt{3}}{12} \beta(\phi) \\
+ & \frac{h^{2}}{8} \nabla^{2} \nabla^{2} \phi\end{aligned}$ & $\begin{array}{c}\frac{h \sqrt{3}}{12} \beta(\phi) \\
+\frac{73 h^{2}}{564} \nabla^{2} \nabla^{2} \phi\end{array}$ & $\begin{array}{c}\frac{h \sqrt{3}}{45} \beta(\phi) \\
+\frac{7 h^{2}}{144} \nabla^{2} \nabla^{2} \phi\end{array}$ \\
\hline $\begin{array}{c}\text { Laplacian } \\
\text { stencil multiplier }\end{array}$ & $\frac{2}{3 h^{2}}$ & $\frac{1}{3 h^{2}}$ & $\frac{1}{267 h^{2}}$ & $\frac{4}{9 h^{2}}$ \\
\hline Family P (1) & -6 & -18 & -504 & -15 \\
\hline Family Q (3) & 0 & -2 & -56 & 3 \\
\hline Family R (6) & 1 & 3 & 69 & 1 \\
\hline Family S (6) & - & 2 & 36 & - \\
\hline Family T (3) & - & -2 & -16 & - \\
\hline Family W (3) & - & - & 30 & - \\
\hline
\end{tabular}

Table 4.1: Stencils and truncation error for Laplacian on a cell-centred mesh 
the geometric weights were set to inverse-distance-squared (distance between cell-reference locations). The second-order least-squares solution for an arbitrary control volume (i,j, $\Delta)$ is

$$
\left(\begin{array}{c}
\phi \\
\frac{\partial \phi}{\partial x} \\
\frac{\partial \phi}{\partial y}
\end{array}\right)=\left(\begin{array}{c}
\bar{\phi}_{(i, j, \Delta)} \\
\frac{\bar{\phi}_{(i, j, \nabla)}-\bar{\phi}_{(i-1, j, \nabla)}}{h} \\
\frac{\bar{\phi}_{(i, j, \nabla)}+\bar{\phi}_{(i-1, j, \nabla)}-2 \bar{\phi}_{(i, j-1, \nabla)}}{h \sqrt{3}}
\end{array}\right) .
$$

An analogous result is obtained for down-pointing triangles. Proceeding to compute the diffusive flux and the single-Gauss-point flux integral as discussed in Section 2.1, we obtain the following expression in terms of control-volume averages for the computed Laplacian in the control volume $(0,0, \Delta)$

$$
\widetilde{\nabla^{2} \phi_{0,0}}=\left(\frac{2}{3 h^{2}}\right)\left(-6 \bar{\phi}_{P}+\Sigma \bar{\phi}_{R}\right)
$$

where $\Sigma \bar{\phi}_{R}$ is the sum of the control-volume averaged solution for all the cells labelled R (six in total) in Figure 4.1.

We can observe that this expression only involves the up-pointing triangle families, $\mathrm{P}$ and $\mathrm{R}$, and as such the solution in the down-pointing triangles are "decoupled" from its up-pointing neighbours. This is analogous to the odd-even decoupling commonly observed in structured meshes. The result of this decoupling can be visualized in Figure 4.2, when we run our numerical solver ANSLib (described in the next chapter) on this uniform equilateral triangle mesh for the Poisson test case. The decoupling clearly affects the solution convergence since the neighbours are not "communicating" with each other. In particular the down-pointing triangles do not have access to the boundary conditions without the help of the up-pointing triangles.

The remedy to this is to break the symmetry that brings about the decoupling or to introduce some form of external coupling. The former is solved by utilizing unstructured meshes while the latter can be achieved with the help of a jump term described in Section 2.1.3.3. The use of the scheme on unstructured meshes still admits the possibility of some decoupling effects, but presence of a jump term should completely negate decoupling 


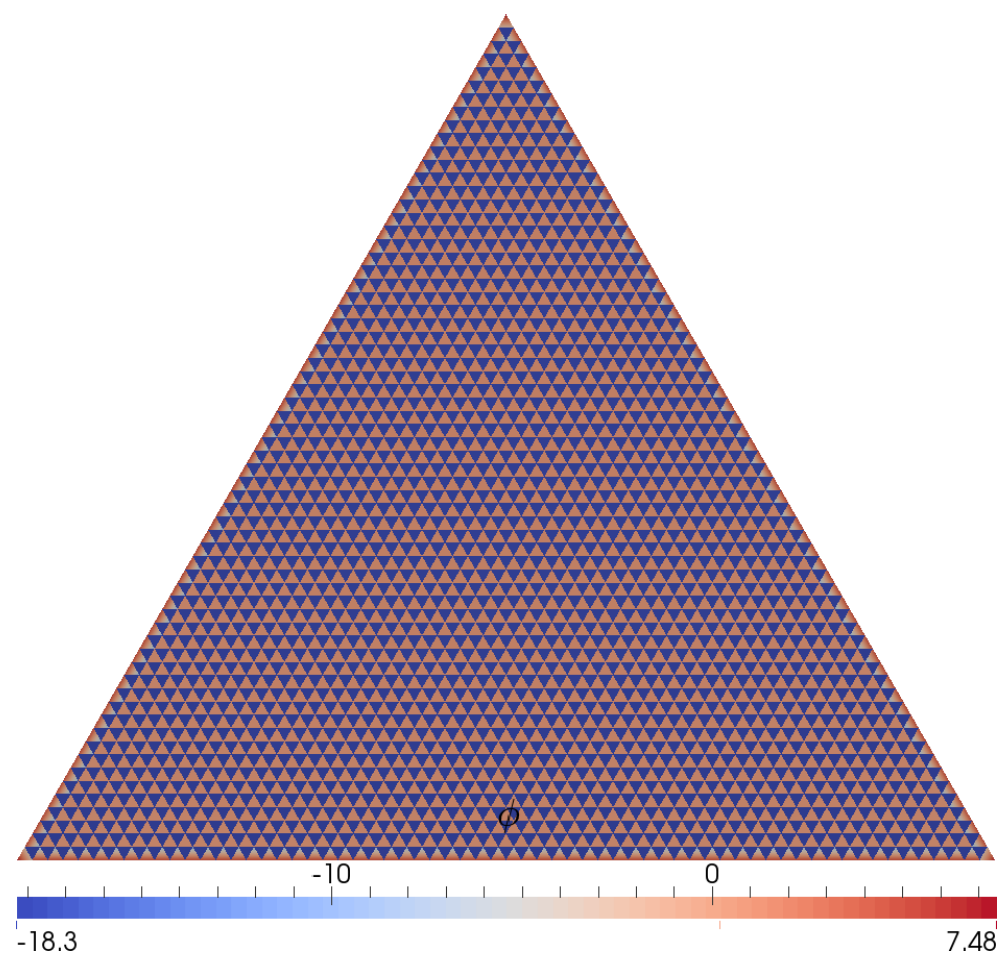

Figure 4.2: Second-order least-squares on uniform equilateral triangle mesh (decoupling)

effects since it directly incorporates the two neighbouring solutions in its formulation. The solution thus obtained is essentially the same as Figure 4.3.

We can expand the control-volume averages in Equation 4.3 in terms of derivatives of the underlying smooth solution $\phi$ at the origin by using Equation 2.17. This gives us the error in the computed Laplacian $\widetilde{\nabla^{2}} \phi_{0,0}$ from the control-volume average of the exact Laplacian $\bar{\nabla}^{2} \phi_{0,0}$ as

$$
{\widetilde{\nabla^{2}}}_{0,0}-\bar{\nabla}^{2} \phi_{0,0}=\frac{h^{2}}{16}\left(\frac{\partial^{4} \phi}{\partial x^{4}}+2 \frac{\partial^{4} \phi}{\partial x^{2} \partial y^{2}}+\frac{\partial^{4} \phi}{\partial y^{4}}\right)+\mathcal{O}\left(h^{4}\right)
$$

The presence of an order- $h^{2}\left(\mathcal{O}\left(h^{2}\right)\right)$ term on the right-hand side shows that this scheme for calculating the Laplacian is second-order accurate on structured cell-centred meshes.

Moreover, by substituting the control-volume average expansions into the gradient formulations in Equation 4.2 it can be easily shown that the first derivatives (gradients) are only first-order accurate. This supports our numerical findings presented in Section 1.1, which was the original motivation for this work. 


\subsubsection{Quadratic Reconstruction}

Proceeding similarly for the (third-order) quadratic reconstruction, all second-layer neighbours (R's) are required to make the least-squares system over-constrained. We apply inverse-distance-squared weighting to these new neighbours. A look at the solution of the third-order least-squares problem in an arbitrary control volume $(\mathrm{i}, \mathrm{j}, \Delta)$, shows the need for the additional layer of neighbours.

$$
\left(\begin{array}{c}
\phi \\
\frac{\partial \phi}{\partial x} \\
\frac{\partial \phi}{\partial y} \\
\frac{\partial^{2} \phi}{\partial x^{2}} \\
\frac{\partial^{2} \phi}{\partial x \partial y} \\
\frac{\partial^{2} \phi}{\partial y^{2}}
\end{array}\right)=\left(\begin{array}{c}
\bar{\phi}_{i, j, \Delta} \\
\frac{\bar{\phi}_{i+1, j, \Delta}-\bar{\phi}_{i-1, j, \Delta}-\bar{\phi}_{i, j-1, \Delta}+\bar{\phi}_{i+1, j-1, \Delta}+3 \bar{\phi}_{i, j, \nabla}-3 \bar{\phi}_{i-1, j, \nabla}}{6 h} \\
\frac{\sqrt{3}\left(2 \bar{\phi}_{i, j+1, \Delta}+2 \bar{\phi}_{i-1, j+1, \Delta}-\bar{\phi}_{i, j-1, \Delta}-\bar{\phi}_{i+1, j-1, \Delta}+3 \bar{\phi}_{i, j, \nabla}+3 \bar{\phi}_{i-1, j, \nabla}-\bar{\phi}_{i+1, j, \Delta}-\bar{\phi}_{i-1, j, \Delta}-6 \bar{\phi}_{i, j-1, \nabla}\right)}{18 h} \\
\frac{-30 \bar{\phi}_{i, j, \Delta}+7 \bar{\phi}_{i+1, j, \Delta}+7 \bar{\phi}_{i-1, j, \Delta}+9 \bar{\phi}_{i, j, \nabla}+9 \bar{\phi}_{i-1, j, \nabla}-2 \bar{\phi}_{i, j+1, \Delta}-2 \bar{\phi}_{i-1, j+1, \Delta}+\bar{\phi}_{i, j-1, \Delta}+\bar{\phi}_{i+1, j-1, \Delta}}{9 h^{2}} \\
\frac{\sqrt{3}\left(2 \bar{\phi}_{i, j+1, \Delta}-2 \bar{\phi}_{i-1, j+1, \Delta}+3 \bar{\phi}_{i, j-1, \Delta}-3 \bar{\phi}_{i+1, j-1, \Delta}+3 \bar{\phi}_{i, j, \nabla}-3 \bar{\phi}_{i-1, j, \nabla}-\bar{\phi}_{i+1, j, \Delta}+\bar{\phi}_{i-1, j, \Delta}\right)}{9 h^{2}} \\
\frac{\bar{\phi}_{i, j-1, \Delta}-\bar{\phi}_{i+1, j, \Delta}-\bar{\phi}_{i-1, j, \Delta}-10 \bar{\phi}_{i, j, \Delta}+2 \bar{\phi}_{i, j+1, \Delta}+2 \bar{\phi}_{i-1, j+1, \Delta}+\bar{\phi}_{i+1, j-1, \Delta}+4 \bar{\phi}_{i, j-1, \nabla}+\bar{\phi}_{i, j, \nabla}+\bar{\phi}_{i-1, j, \nabla}}{3 h^{2}}
\end{array}\right)
$$

Once again we proceed to find the diffusive fluxes, which now incorporate the secondderivative terms, and the flux integral using a two-point Gauss quadrature. This gives us the following expression in terms of control-volume averages for the computed Laplacian in the control volume $(0,0, \Delta)$

$$
\widetilde{\nabla^{2} \phi_{0,0}}=\left(\frac{4}{9 h^{2}}\right)\left(-15 \bar{\phi}_{P}+3 \Sigma \bar{\phi}_{Q}+\Sigma \bar{\phi}_{R}\right)
$$

where, for example, $\Sigma \bar{\phi}_{Q}$ is the sum of the control-volume averaged solution for all the cells labelled Q (three in total) in Figure 4.1.

Equation 4.6 incorporates both the up as well as the down-pointing triangles, unlike the second-order version seen in Equation 4.3. Thus we can expect this third-order leastsquares scheme to not be decoupled. This is verified by the successful convergence of our numerical simulation of this reconstruction scheme on an equilateral triangle mesh, shown 


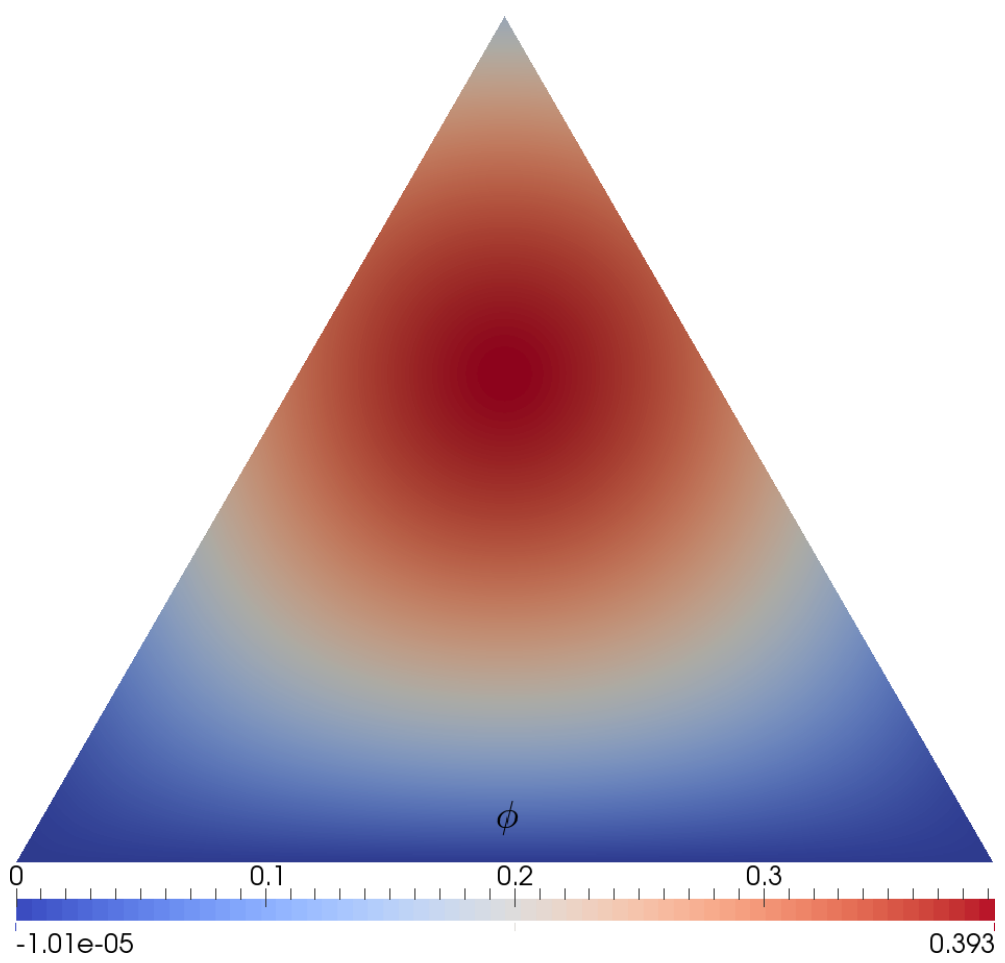

Figure 4.3: Third-order least-squares on uniform equilateral triangle mesh

in Figure 4.3.

Upon substituting the respective expansions of the control-volume averages from Equation 2.17 into Equation 4.6, we can find its exact error and its order of accuracy. The error in the Laplacian for third-order least-squares is given by

$$
\widetilde{\nabla^{2} \phi_{0,0}}-\bar{\nabla}^{2} \phi_{0,0}=\frac{h \sqrt{3}}{45}\left(3 \frac{\partial^{3} \phi}{\partial x^{2} \partial y}-\frac{\partial^{3} \phi}{\partial y^{3}}\right)+\frac{7 h^{2}}{144}\left(\frac{\partial^{4} \phi}{\partial x^{4}}+2 \frac{\partial^{4} \phi}{\partial x^{2} \partial y^{2}}+\frac{\partial^{4} \phi}{\partial y^{4}}\right)+\mathcal{O}\left(h^{3}\right) .
$$

This is a surprising result, since the Laplacian is only first-order accurate here due to the presence of an $\mathcal{O}(h)$ term on the right-hand side. Moreover, even if we were to ignore this first-order term, we still have to contend with the second-order accurate term which is only slightly smaller than its counter-part in the linear reconstruction. As explained in [43] this first-order term is an unfortunate combination of third-derivative terms that cannot be computed independently for any single family of control volumes. This can be primarily attributed to the reconstruction's symmetric stencils being unable to distinguish between the non-zero $x^{2} y$ and $y^{3}$ moments in the Taylor series expansion of the control-volume averages on uniform triangular meshes. However, fortunately this does not extend to unstructured 
meshes where, as we have seen in Figure 1.3b, the third-order (quadratic) least-squares reconstruction performs much better, thanks to the absence of this fatal symmetry.

\subsection{3 $\quad \mathrm{H}_{1}$ Reconstruction}

For our proposed $\mathrm{H}_{1}$ reconstruction we present two cases, one with a regular quadratic stencil ( $\mathrm{Q}+\mathrm{R}$ families) and the other with an extended stencil which encompasses all the vertex neighbours of the given control volume $(\mathrm{Q}+\mathrm{R}+\mathrm{T}$ families $)$. This provides us with insights into how our scheme is affected by extending the reconstruction stencil.

\subsubsection{Regular Stencil $\mathrm{H}_{1}$ Reconstruction}

The reconstruction stencil used for our proposed $\mathrm{H}_{1}$ reconstruction is the same as the one used for the quadratic reconstruction. Thus the reconstruction solution we obtain is also similar save that we do not obtain the second-degree derivatives, as given by:

$$
\left(\begin{array}{c}
\phi \\
\frac{\partial \phi}{\partial x} \\
\frac{\partial \phi}{\partial y}
\end{array}\right)=\left(\begin{array}{c}
\bar{\phi}_{i, j, \Delta} \\
\frac{\bar{\phi}_{i+1, j, \Delta}-\bar{\phi}_{i-1, j, \Delta}-\bar{\phi}_{i, j-1, \Delta}+\bar{\phi}_{i+1, j-1, \Delta}+3 \bar{\phi}_{i, j, \nabla}-3 \bar{\phi}_{i-1, j, \nabla}}{6 h} \\
\frac{2\left(\bar{\phi}_{i, j+1, \Delta}+\bar{\phi}_{i-1, j+1, \Delta}\right)-\bar{\phi}_{i, j-1, \Delta}-\bar{\phi}_{i+1, j-1, \Delta}+3\left(\bar{\phi}_{i, j, \nabla}+\bar{\phi}_{i-1, j, \nabla}\right)-\bar{\phi}_{i+1, j, \Delta}-\bar{\phi}_{i-1, j, \Delta}-6 \bar{\phi}_{i, j-1, \nabla}}{6 h \sqrt{3}}
\end{array}\right)
$$

Using a two-point Gauss quadrature, we obtain the following expression for the computed Laplacian in the control volume $(0,0, \Delta)$

$$
\widetilde{\nabla^{2} \phi_{0,0}}=\left(\frac{1}{3 h^{2}}\right)\left(-18 \bar{\phi}_{P}-2 \Sigma \bar{\phi}_{Q}+3 \Sigma \bar{\phi}_{R}+2 \Sigma \bar{\phi}_{S}-2 \Sigma \bar{\phi}_{T}\right)
$$

in terms of the control-volume averages and

$$
\widetilde{\nabla^{2} \phi_{0,0}}-\bar{\nabla}^{2} \phi_{0,0}=\frac{h \sqrt{3}}{12}\left(3 \frac{\partial^{3} \phi}{\partial x^{2} \partial y}-\frac{\partial^{3} \phi}{\partial y^{3}}\right)+\frac{h^{2}}{8}\left(\frac{\partial^{4} \phi}{\partial x^{4}}+2 \frac{\partial^{4} \phi}{\partial x^{2} \partial y^{2}}+\frac{\partial^{4} \phi}{\partial y^{4}}\right)+\mathcal{O}\left(h^{3}\right)
$$

as the error in the computed Laplacian. 


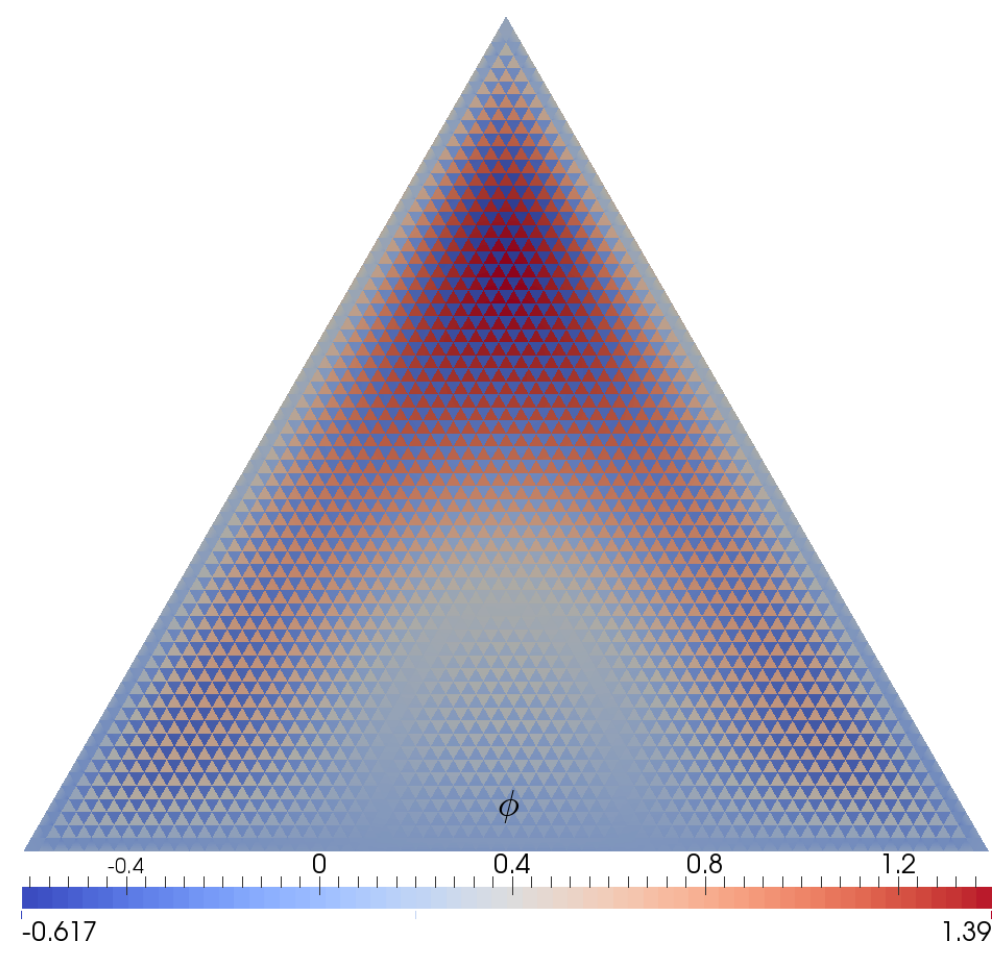

Figure 4.4: Regular $\mathrm{H}_{1}$ reconstruction on uniform equilateral triangle mesh (decoupling)

We can instantly recognize the similarities with the parent quadratic reconstruction, from which $\mathrm{H}_{1}$ reconstruction is derived, especially with a similar first-order term in the error expression for Laplacian, albeit larger by almost a constant factor of 4 . The second-order term seems larger than even its counter-part in linear reconstruction. However, fortunately the scheme fares better when used with realistic unstructured meshes, as we will see in Chapter 5.

Another anomaly we observed was an unforeseen pattern of solution decoupling, as seen in Figure 4.4, when numerical simulations were conducted on the uniform equilateral mesh. However this is not supported by our understanding of Equation 4.9, which shows no signs of decoupling as was evident in Equation 4.3 for the linear reconstruction scheme. Once again, this decoupling can be countered with the aid of a jump term, just like in the linear reconstruction case. 


\subsubsection{Extended Stencil $\mathrm{H}_{1}$ Reconstruction}

For the extended stencil $\mathrm{H}_{1}$ Reconstruction, we include all the vertex neighbours of the given control volume, namely the $\mathrm{Q}, \mathrm{R}$ and $\mathrm{T}$ family of cells seen in Figure 4.1. We include just three additional neighbours ( $\mathrm{T}$ family) instead of the entire third-layer of neighbours which includes the S family of neighbours as well. The reconstruction solution we get is given by:

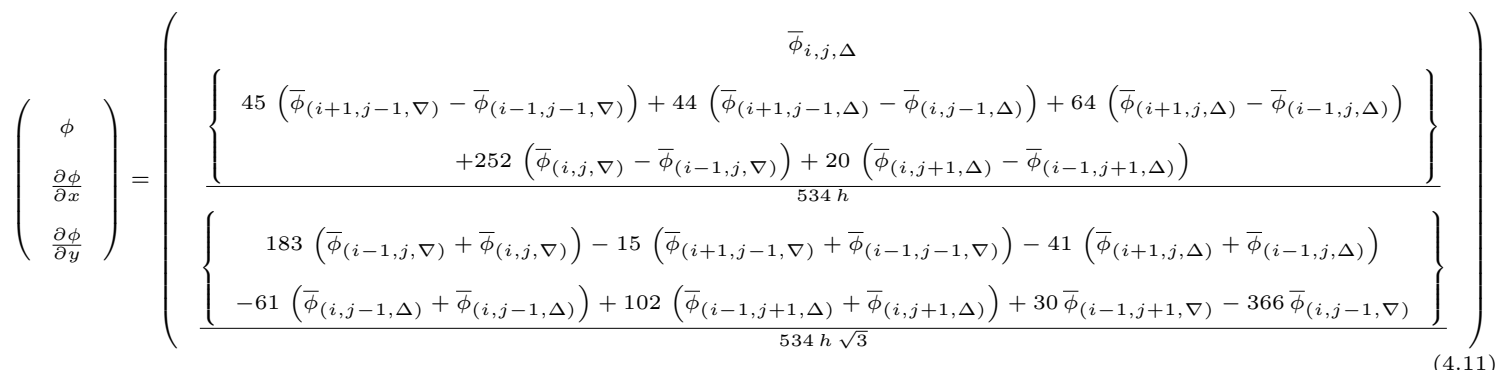

Once again using a two-point Gauss quadrature, we obtain the following expression for the computed Laplacian in the control volume $(0,0, \Delta)$

$$
\widetilde{\nabla^{2} \phi_{0,0}}=\left(\frac{1}{267 h^{2}}\right)\left(-504 \bar{\phi}_{P}-56 \Sigma \bar{\phi}_{Q}+69 \Sigma \bar{\phi}_{R}+36 \Sigma \bar{\phi}_{S}-16 \Sigma \bar{\phi}_{T}+30 \Sigma \bar{\phi}_{U}\right)
$$

in terms of the control-volume averages and

$$
{\widetilde{\nabla^{2}} \phi_{0,0}}-\bar{\nabla}^{2} \phi_{0,0}=\frac{h \sqrt{3}}{12}\left(3 \frac{\partial^{3} \phi}{\partial x^{2} \partial y}-\frac{\partial^{3} \phi}{\partial y^{3}}\right)+\frac{287 h^{2}}{2136}\left(\frac{\partial^{4} \phi}{\partial x^{4}}+2 \frac{\partial^{4} \phi}{\partial x^{2} \partial y^{2}}+\frac{\partial^{4} \phi}{\partial y^{4}}\right)+\mathcal{O}\left(h^{3}\right)
$$

as the error in the computed Laplacian.

Here too we find the same decoupling quirk as seen with the regular case, as observed in Figure 4.5.

It is apparent that an extension of the stencil does not have much impact on the accuracy of the system, however we have observed a slight increase in stability of the system with the extension of the stencil during numerical simulation using our flow-solver ANSLib. However the additional stability was negligible compared to the effects of other measures such as the use of a jump term. Moreover the added benefits do not justify the more 


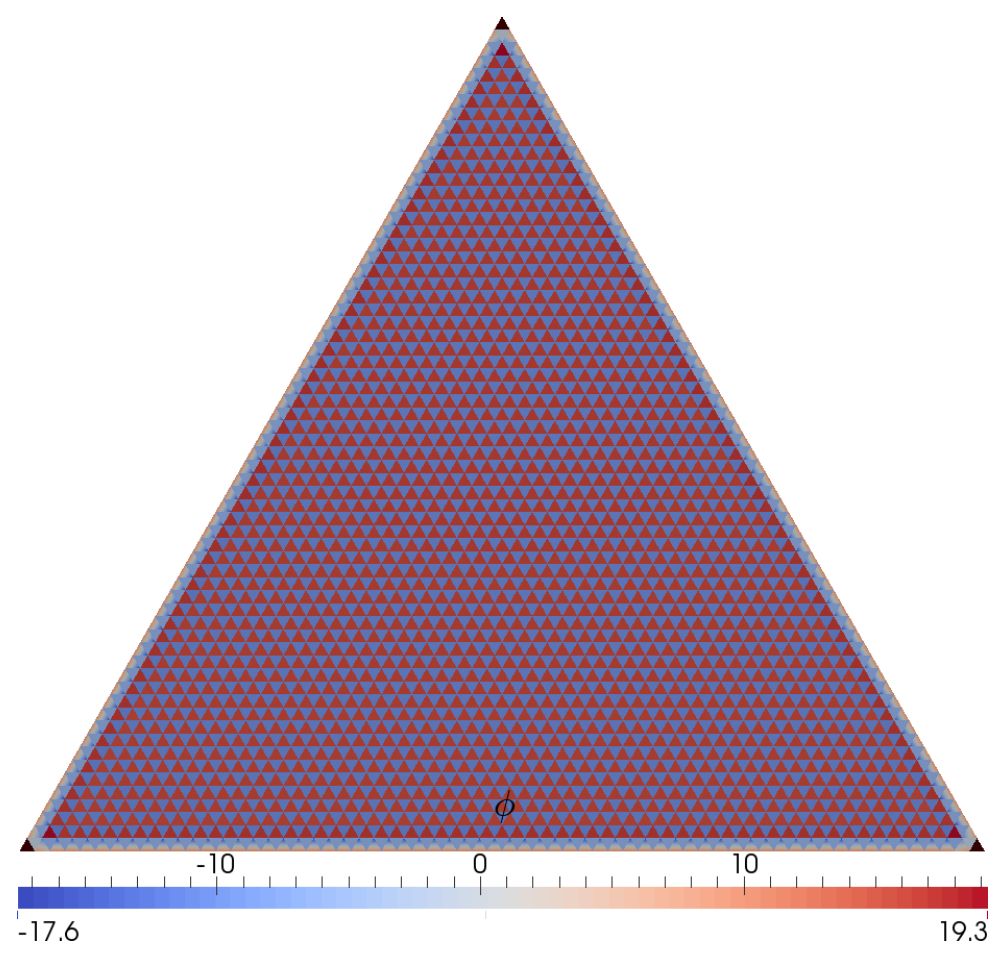

Figure 4.5: Extended $\mathrm{H}_{1}$ reconstruction on uniform equilateral triangle mesh (decoupling)

intensive computations required due to the addition of these additional vertex neighbours (and consequently, additional rows in the left-hand side of every control-volume's least squares system).

Henceforth, we will stick to the regular $\mathrm{H}_{1}$ reconstruction for all of our numerical tests and simulations that follow in the next chapter. 


\section{Chapter 5}

\section{Numerical Tests and Analysis}

The analytic tests in the previous chapter were designed for meshes with regular topology to provide insights into the behaviour of the discretization schemes on a structured mesh. Similar analysis on an unstructured mesh would prove highly impractical for every control volume would have its own unique qualities and reconstruction stencil. Here instead we compare and analyze the various accuracy results from our proposed $\mathrm{H}_{1}$ finite-volume scheme and the conventional second and third order least-squares reconstruction schemes. All the numerical experiments were conducted using the Advanced Numerical Simulations Library (ANSLib), the unstructured numerical simulation code developed in Advanced Numerical Simulations Laboratory (ANSLab), UBC.

The precise and consistent evaluation of the numerical errors generated is essential to analyze the accuracy of any given scheme. The discretization error, which forms the majority of the numerical error, along with its primary source, the truncation error, can be estimated by a technique known as the method of manufactured solutions (MMS). This technique has not only been implemented to estimate the numerical error but also used for order of convergence studies and code verification [54].

In MMS, an exact solution that satisfies the given governing equations is known (or manipulated using a source term) and preset. Hence errors can be computed by evaluating and comparing the final discrete solution obtained through the simulation with this exact solution. The same is done for the corresponding flux and flux integrals. The $L_{2^{-}}$ norm of these errors are then evaluated for a series of five consistently-refined cell-centred unstructured meshes and analyzed to obtain the asymptotic order of convergence of the scheme. The mesh refinement was performed by reducing the length scale by a factor of two to form the next finer mesh. The triangular meshes are regular isotropic and non-nested 


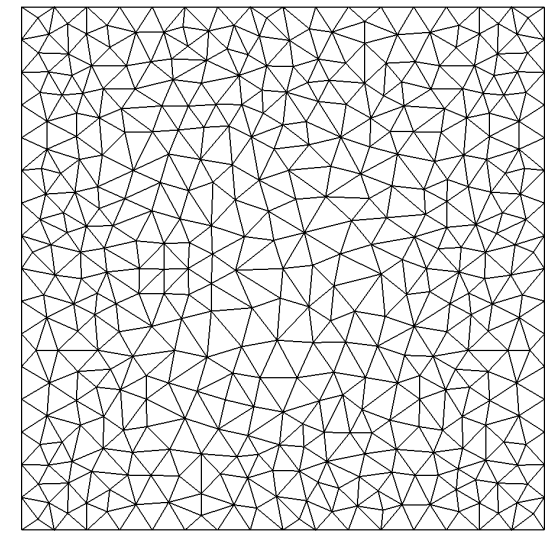

(a) Square mesh with 780 triangles

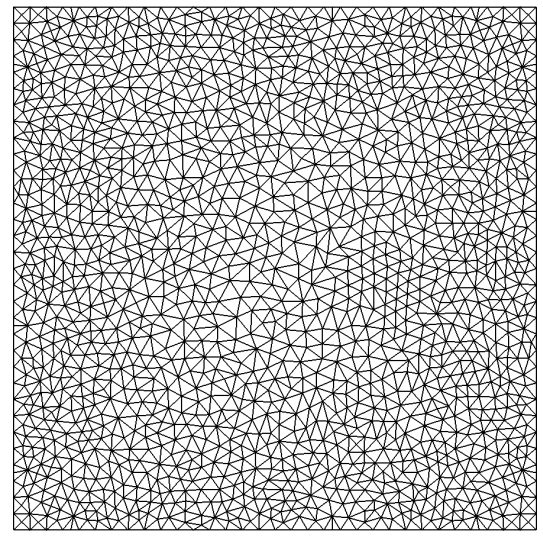

(b) Square mesh with 3132 triangles

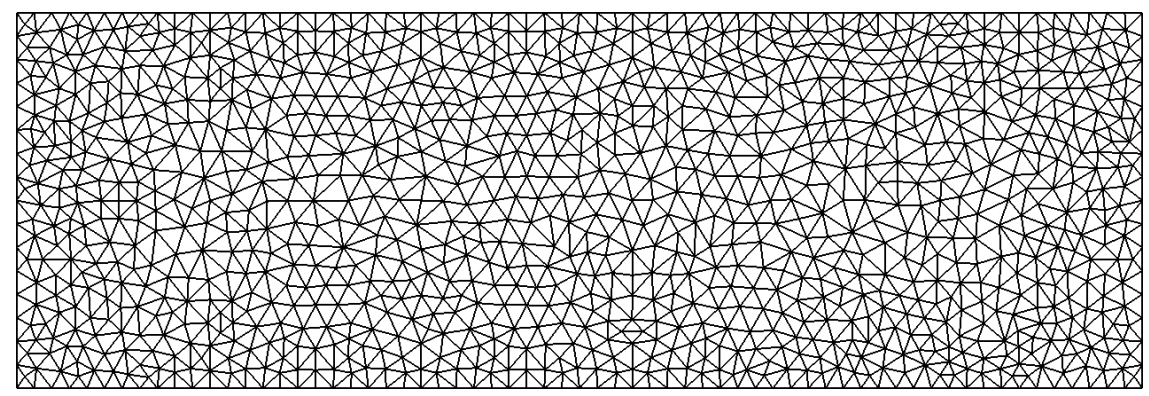

(c) Channel mesh with 2162 triangles

Figure 5.1: Isotropic unstructured meshes used

meshes and were generated by Delaunay refinement. Figure 5.1 shows a few of the isotropic unstructured meshes used.

We begin with some preliminary studies, which verify the foundations of our new $\mathrm{H}_{1}$ finite-volume scheme. Complete accuracy analysis was then performed for two separate model problems, namely the Poisson equation and the advection-diffusion equation.

\subsection{Preliminary Studies}

\subsubsection{One-dimensional $\mathbf{H}_{1}$ Flux Discretization}

Preliminary studies were undertaken to verify the advantages of $\mathrm{H}_{1}$ flux discretization in onedimension that was described in subsection 3.2.1. A Matlab code was written to compare the accuracy achieved with the use of the simple arithmetic averaging as well as the linear interpolation given by Equations 3.6 and 3.7 respectively, assuming second-order gradients at the cell reference points. The tests were conducted on a series of randomly perturbed 


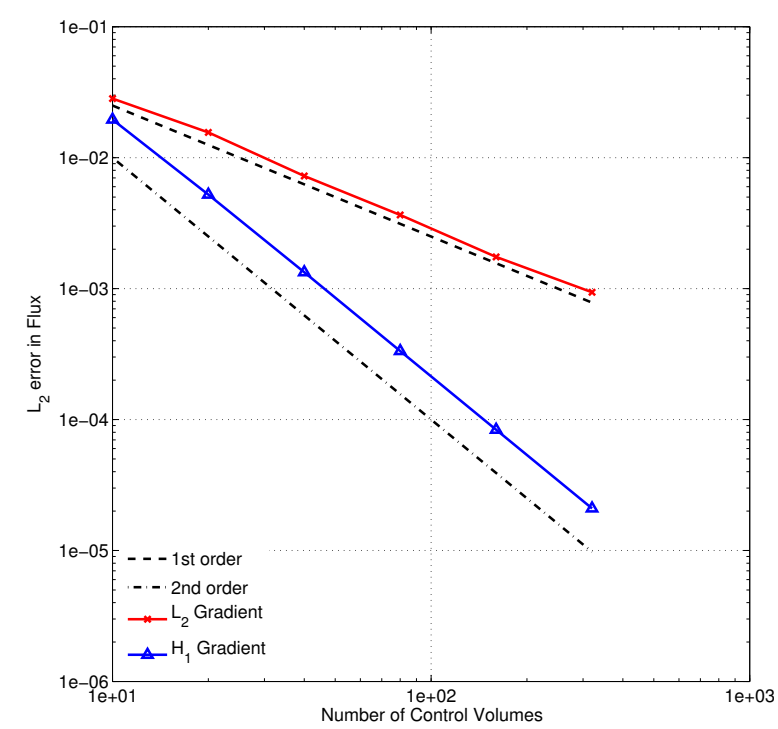

(a) One-dimensional $\mathrm{H}_{1}$ flux discretization

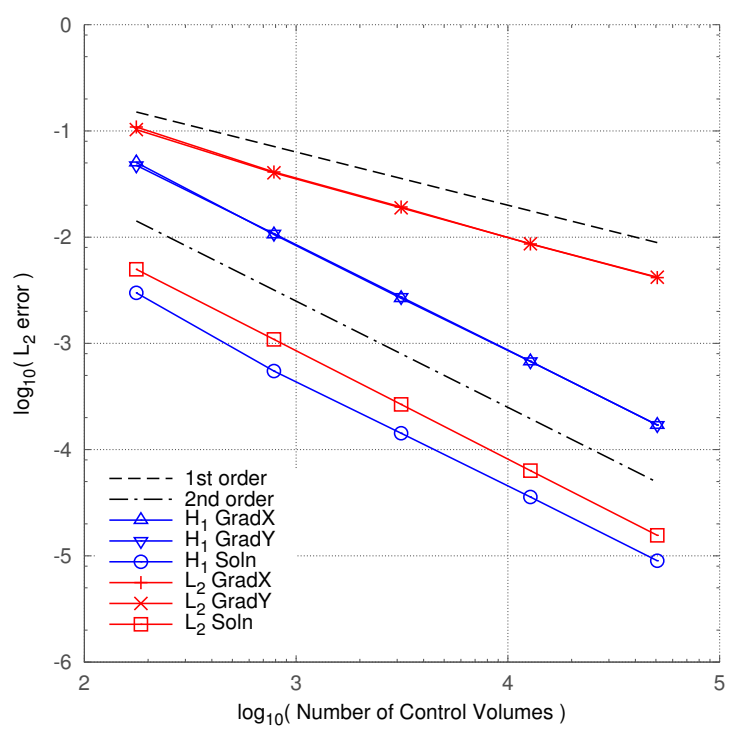

(b) Order of reconstruction accuracy in two dimensions

Figure 5.2: Preliminary Studies

one-dimensional grids with the cell mid-side as the cell reference point. A test function of $e^{-x^{2}}$ was used as the manufactured solution with Dirichlet boundary conditions.

Figure 5.2a shows the results of the preliminary tests in one-dimension, which verifies our theory. The linear interpolation of $\mathrm{H}_{1}$ reconstruction does indeed provide us with second-order gradients at the cell interface in contrast to the first-order gradients obtained with simple arithmetic averaging of second-order least-squares scheme.

\subsubsection{Two-dimensional $\mathrm{H}_{1}$ Reconstruction Tests}

The consistency of the $\mathrm{H}_{1}$ reconstruction in two-dimensions, detailed in section 3.1, was also verified. A manufactured solution test function of $\frac{\pi}{8} \sin (\pi x) \sin (\pi y)$ was used to assess the accuracy of the reconstruction, with homogeneous Dirichlet boundary condition enforcement.

Figure $5.2 \mathrm{~b}$ compares the reconstruction order of accuracy of our new $\mathrm{H}_{1}$ reconstruction with that of second-order least-squares reconstruction for the solution and the two gradient components.

As is clear, we have been successful in increasing the order of accuracy of the reconstructed gradients to second order using $\mathrm{H}_{1}$ reconstruction while the same are first-order 


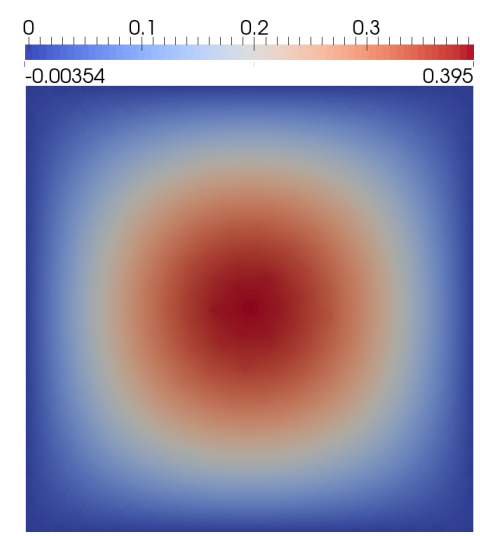

(a) Case (i) $\frac{\pi}{8} \sin (\pi x) \sin (\pi y)$

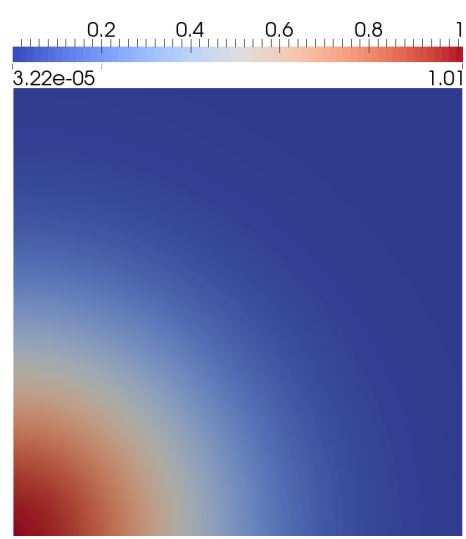

(b) Case (ii) Gaussian Bump: $e^{-\left(x^{2}+y^{2}\right) / 0.2}$

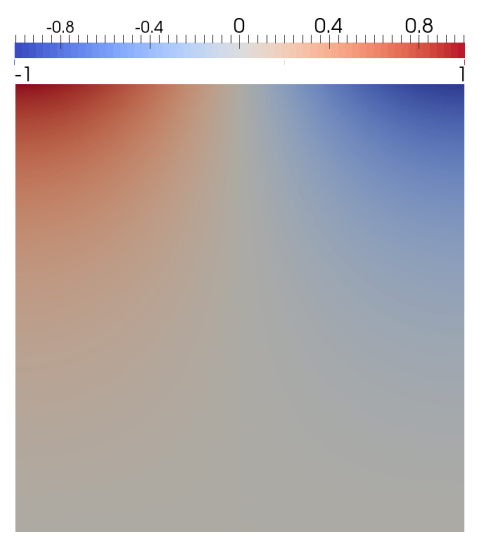

(c) Case (iii) Plain Laplacian: $\frac{\cos (\pi x) \sinh (\pi y)}{\sinh (\pi)}$

Figure 5.3: Manufactured solutions for Poisson equation on an unstructured mesh

accurate in second-order (linear) least-squares reconstruction.

\subsection{D Poisson Test}

The Poisson equation serves as the ideal scalar model of diffusion problems. Three unique manufactured solution test functions were used to assess the accuracy of the reconstruction, namely:

Case (i) Sinusoidal: $\frac{\pi}{8} \sin (\pi x) \sin (\pi y)$, with Dirichlet boundary conditions

Case (ii) Gaussian Bump: $\exp \left(-\left(x^{2}+y^{2}\right) / 0.2\right)$, with Dirichlet boundary conditions

Case (iii) Plain Laplacian: $\cos (\pi x) \sinh (\pi y) / \sinh (\pi)$, with Dirichlet boundaries on the top and bottom walls and Neumann boundaries on the side walls

Figure 5.3 shows the resultant manufactured solutions on a unit square mesh consisting of 780 control volumes, with the origin located at the bottom left corner.

The orders of accuracy of the Poisson solution, flux and flux integral have been plotted and compared in Figures 5.4, 5.5 and 5.6. The tests were run on 5 successively refined meshes containing 176, 780, 3132, 12766 and 50715 isotropic triangles.

The diffusive flux at the Gauss points using the smooth initial data is second-order accurate as seen in Figures 5.4a, 5.5a and 5.6a. 


\begin{tabular}{|c|c|c|c|c|c|c|c|c|c|}
\hline \multirow{2}{*}{ Quantity } & \multicolumn{3}{|c|}{$\begin{array}{c}\text { Second-order } \\
\text { least squares }\end{array}$} & \multicolumn{3}{c|}{$\mathrm{H}_{1}$} & \multicolumn{3}{c|}{ Third-order } \\
reconstruction \\
\cline { 2 - 11 } & $\begin{array}{c}\text { Case } \\
\text { (i) }\end{array}$ & $\begin{array}{c}\text { Case } \\
\text { (ii) }\end{array}$ & $\begin{array}{c}\text { Case } \\
\text { (iii) }\end{array}$ & $\begin{array}{c}\text { Case } \\
\text { (i) }\end{array}$ & $\begin{array}{c}\text { Case } \\
\text { (ii) }\end{array}$ & $\begin{array}{c}\text { Case } \\
(\mathrm{iii})\end{array}$ & $\begin{array}{c}\text { Case } \\
\text { (i) }\end{array}$ & $\begin{array}{c}\text { Case } \\
\text { (ii) }\end{array}$ & $\begin{array}{c}\text { Case } \\
(\mathrm{iii})\end{array}$ \\
\hline \hline $\begin{array}{c}\text { Flux } \\
\text { (initial data) }\end{array}$ & 1.14 & 1.07 & 1.21 & 1.82 & 2.01 & 1.73 & 2.06 & 2.10 & 2.22 \\
\hline $\begin{array}{c}\text { Flux integral } \\
\text { (initial data) }\end{array}$ & 0.01 & 0.11 & 0.14 & 0.62 & 0.56 & 0.47 & 1.36 & 1.20 & 1.10 \\
\hline $\begin{array}{c}\text { Flux } \\
\text { (converged) }\end{array}$ & 1.22 & 1.38 & 1.41 & 1.53 & 1.58 & 1.32 & 2.05 & 2.11 & 2.20 \\
\hline Solution & 2.17 & 2.03 & 2.31 & 2.02 & 2.08 & 2.05 & 2.05 & 2.01 & 3.00 \\
\hline
\end{tabular}

Table 5.1: Orders of accuracy for Poisson model problem

With the flux-integral order obtained at this stage, as seen in Figures 5.4b, 5.5b and 5.6b, we can observe that $\mathrm{H}_{1}$ reconstruction is indeed first order accurate but loses accuracy on the finest mesh. None the less it still shows much better accuracy compared to a secondorder least-squares method. Note that this flux integral was still computed by using just a single Gauss quadrature point.

When the solution is fully converged, the flux shows a trend similar to the flux integral, losing its order as it progresses to finer meshes; this is illustrated by Figures 5.4c, 5.5c and 5.6c.

As for the converged solution itself, seen in Figures 5.4d, 5.5d and 5.6d, all three schemes are comparable and essentially produce second-order accurate solutions.

The orders of accuracy achieved for the solution, diffusive flux and the flux integral with each scheme and for all cases, are tabulated in Table 5.1. The values listed are the averaged rates of convergence across all the five refinement levels.

Another interesting feature of the $\mathrm{H}_{1}$ reconstruction can be found when we consider its solution error. When compared to the other least-squares schemes, seen in Figure 5.7 for the test case (i), it is able to overcome the rough noise plaguing the second-order least-squares discretization error and is closer to a third-order least-squares in terms of its smoothness. Overall, the differences in the error distribution for the three schemes are quite striking, considering that the error norms are nearly identical. 


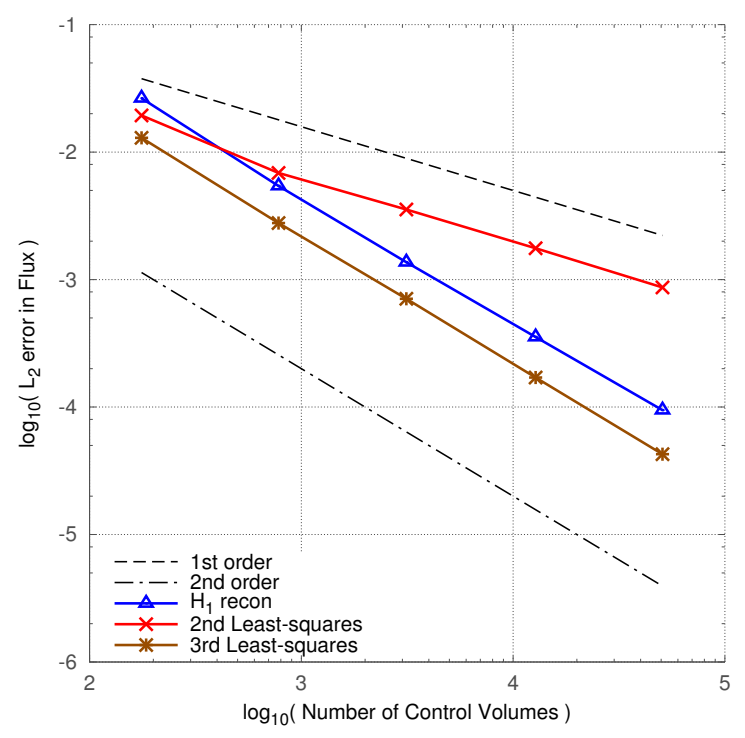

(a) Flux convergence rate at Gauss points (initial data)

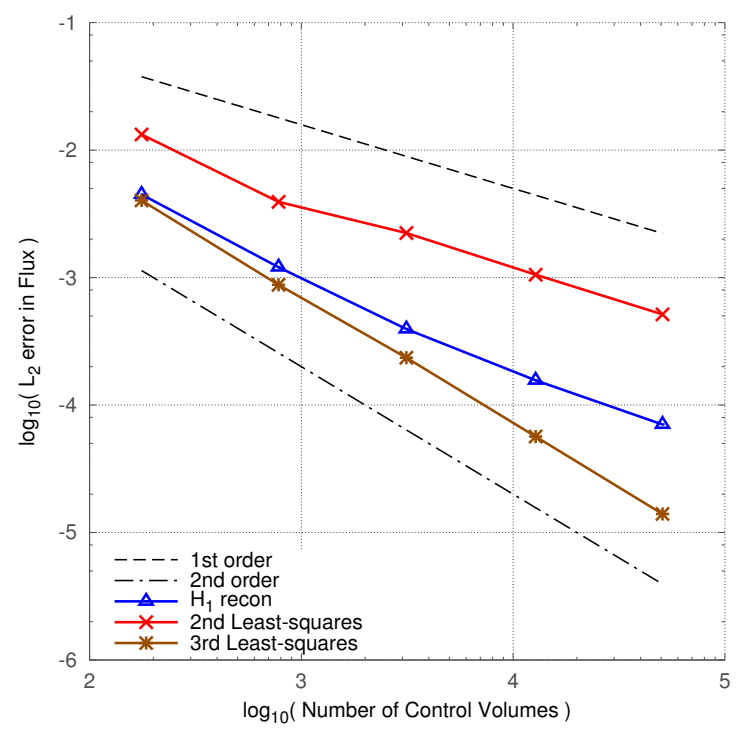

(c) Flux convergence rate at Gauss points (converged solution)

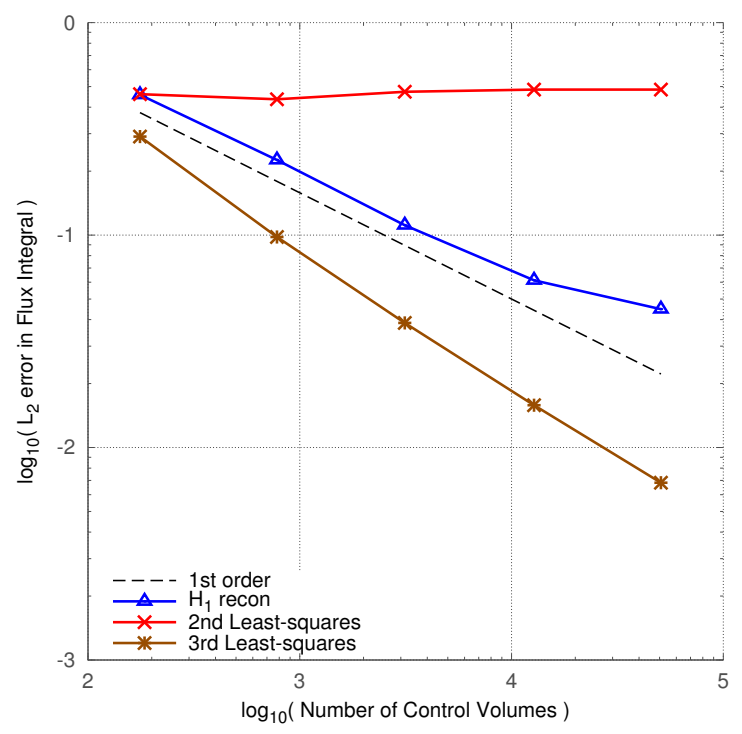

(b) Flux integral convergence rate (initial data)

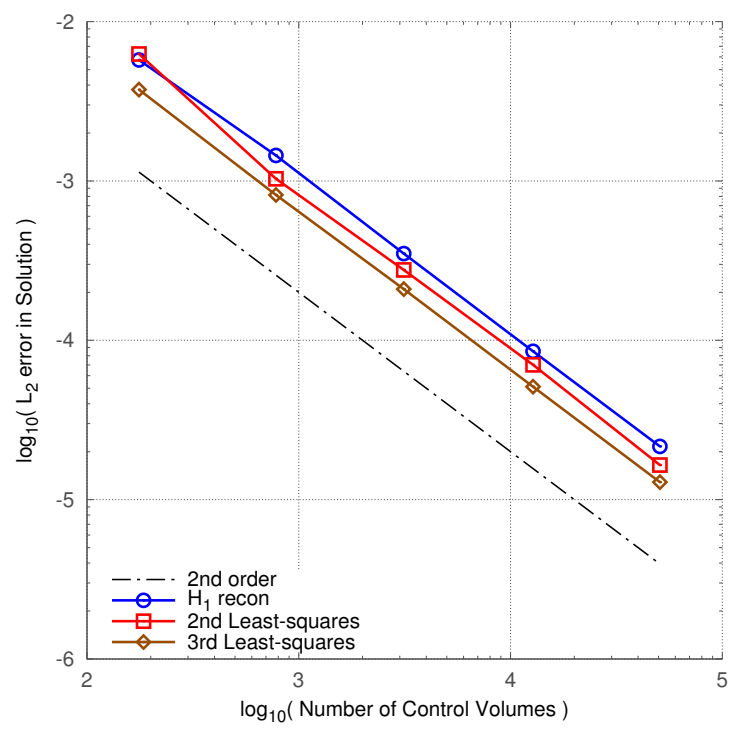

(d) Solution convergence rate

Figure 5.4: Case (i) Sinusoidal Poisson 2D test results 


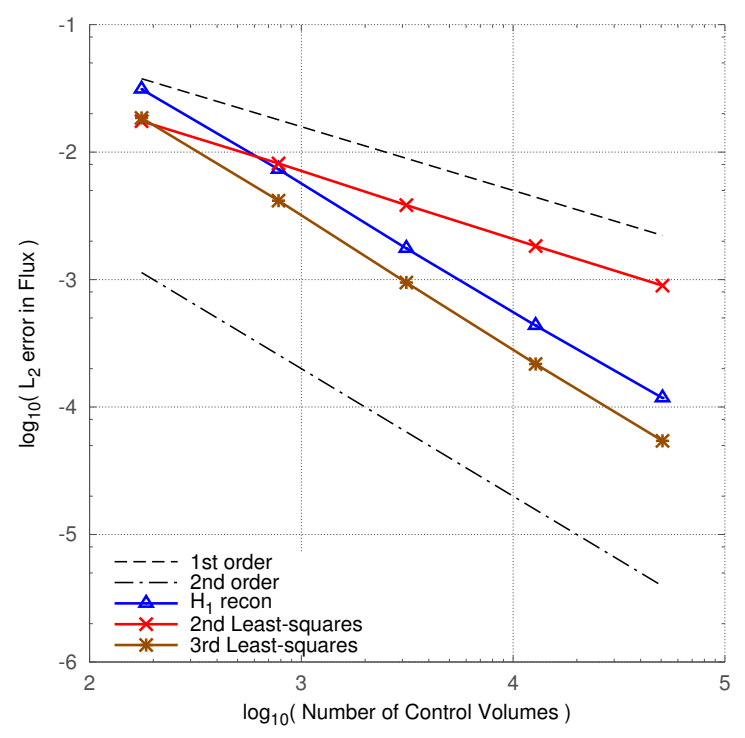

(a) Flux convergence rate at Gauss points (initial data)

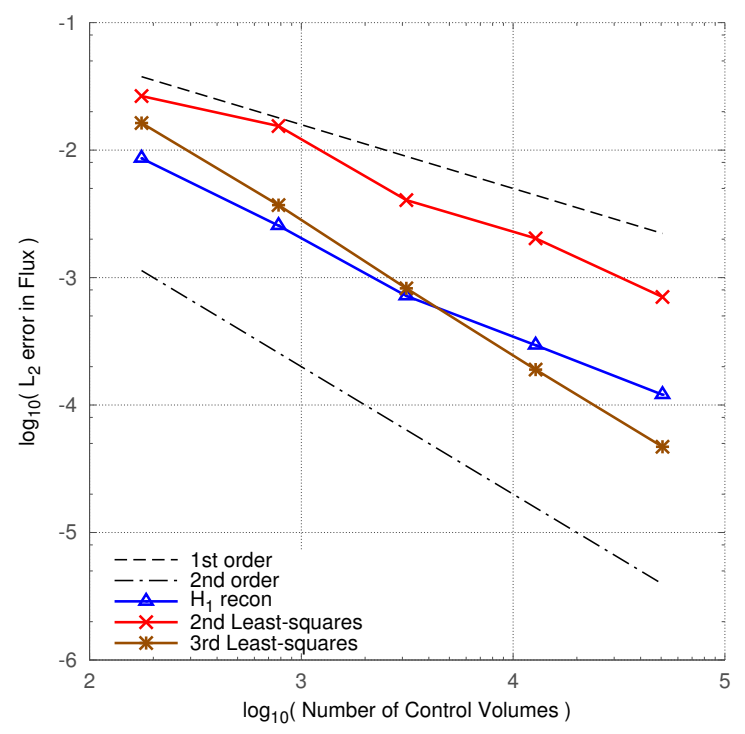

(c) Flux convergence rate at Gauss points (converged solution)

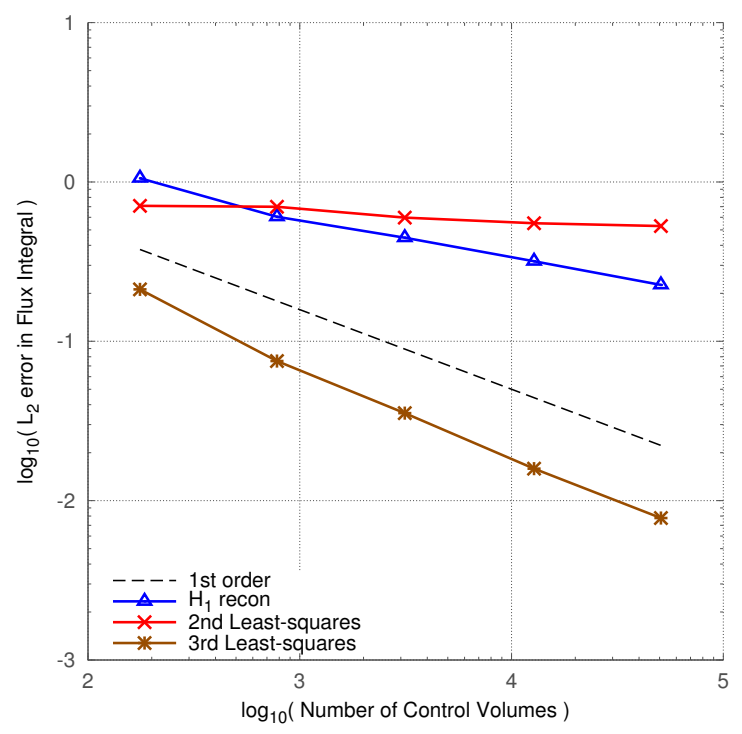

(b) Flux integral convergence rate (initial data)

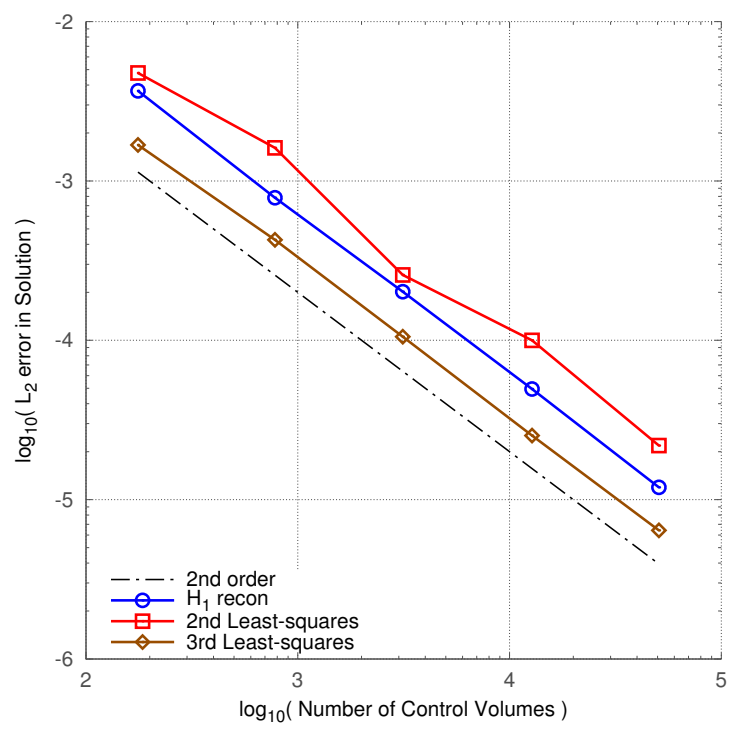

(d) Solution convergence rate

Figure 5.5: Case (ii) Gaussian Bump Poisson 2D test results 


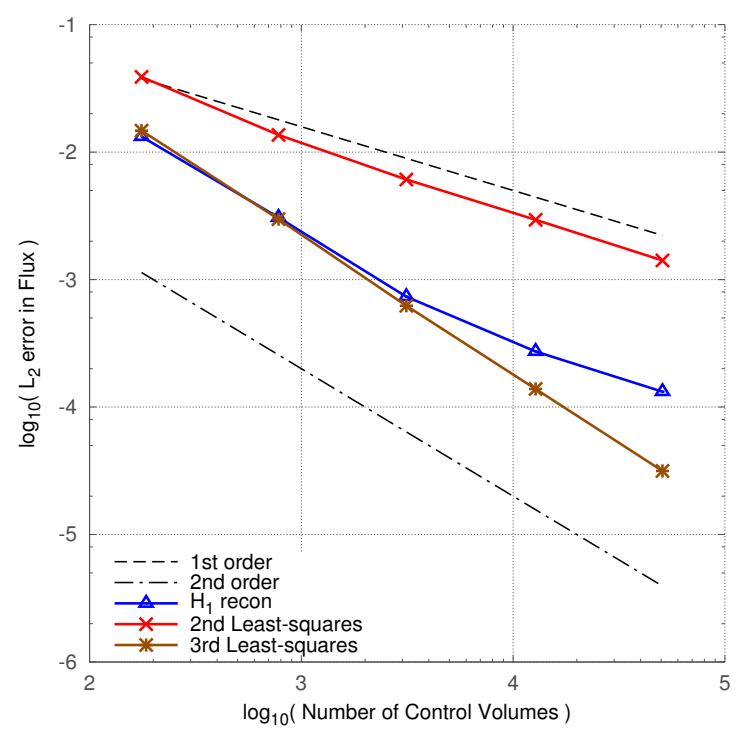

(a) Flux convergence rate at Gauss points (initial data)

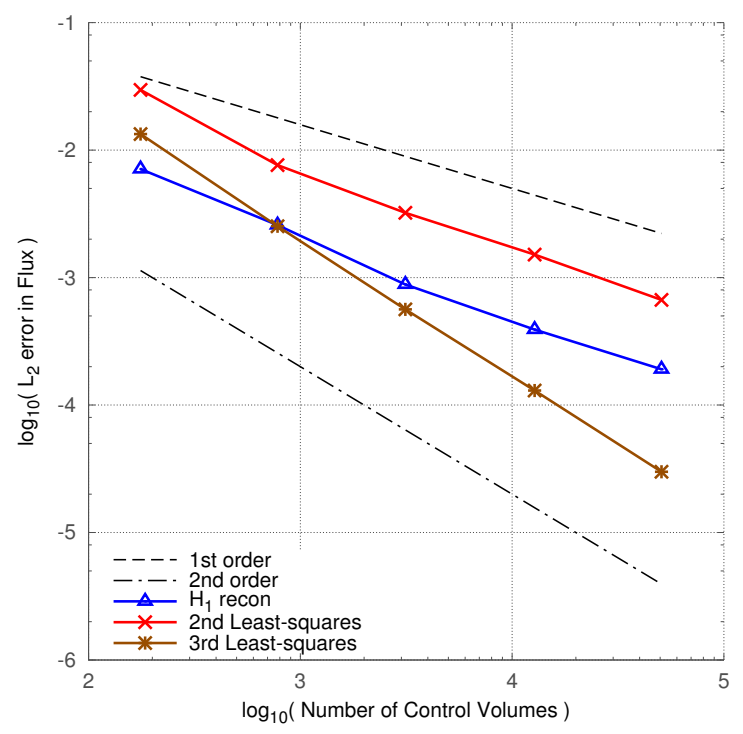

(c) Flux convergence rate at Gauss points (converged solution)

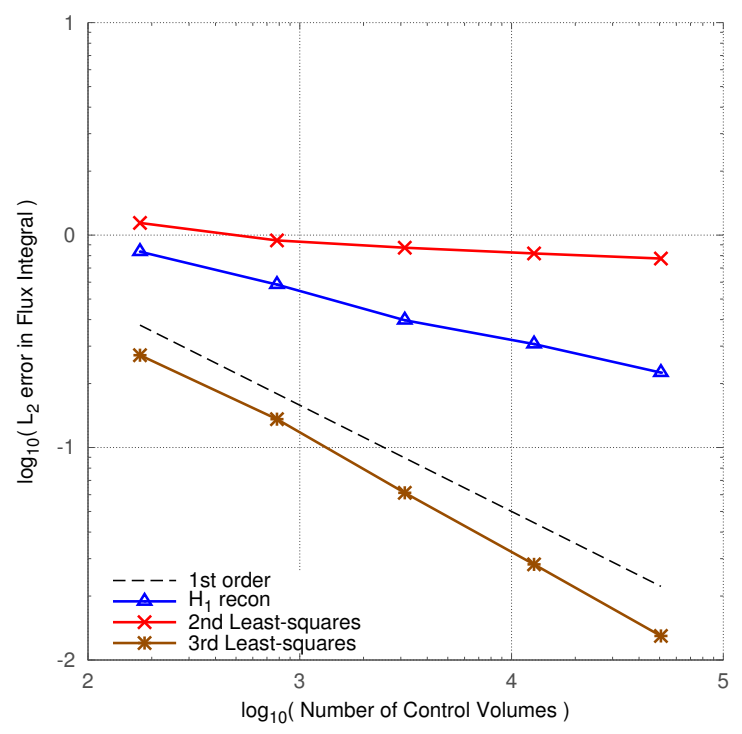

(b) Flux integral convergence rate (initial data)

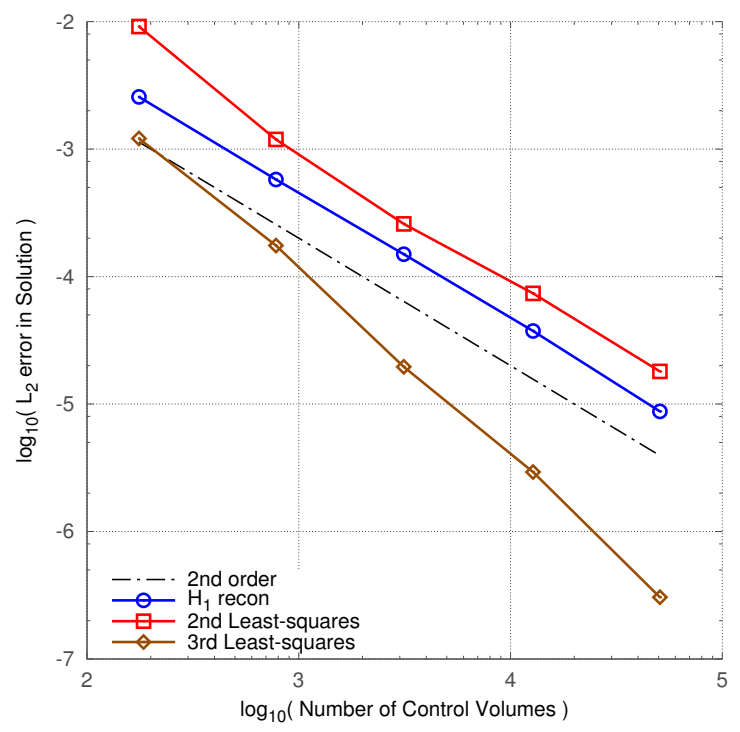

(d) Solution convergence rate

Figure 5.6: Case (iii) Plain Laplacian Poisson 2D test results 


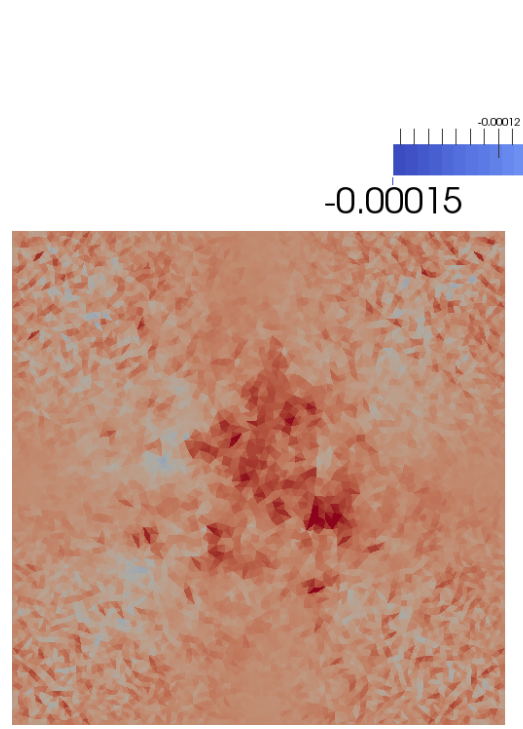

(a) Second-order least-squares

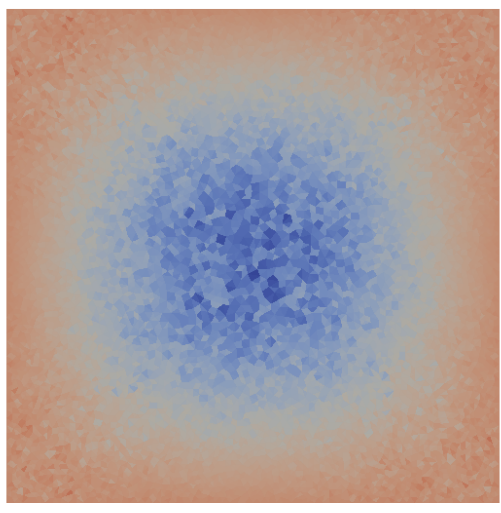

(b) $\mathrm{H}_{1}$ reconstruction
$4 e-5$

7e-05

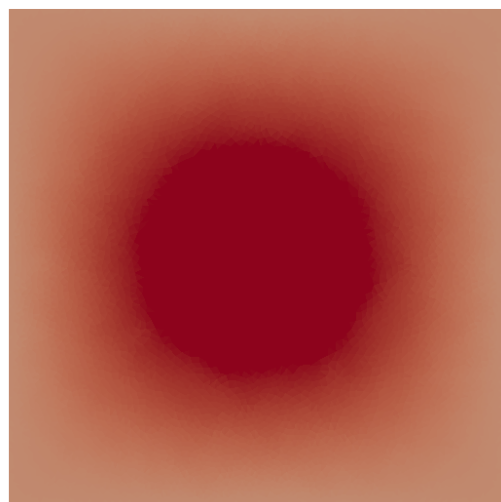

(c) Third order least-squares

Figure 5.7: Solution error comparison

\subsection{D Advection-Diffusion Test}

As a further demonstration of $\mathrm{H}_{1}$ reconstruction, we solve the advection-diffusion equation

$$
u \cdot \nabla \phi=\alpha \nabla^{2} \phi
$$

where $\alpha$ is the diffusion coefficient and $u$ is the velocity vector. The advection-diffusion equation is a model equation which is closer to real world flows, serving as a preliminary step towards future applications to the Navier-Stokes equations. The inherent stability of the upwind discretization of the convective terms is an overall benefit to the stability of this problem using $\mathrm{H}_{1}$ reconstruction. We use the same $\mathrm{H}_{1}$ reconstruction to compute the convective terms as we use for the diffusive terms. The tests were conducted on a series of unstructured meshes in a rectangular channel of height $(\mathrm{H}) 1$ and length (L) 3, initialized with smooth exact solution data. The top and bottom wall were enforced with homogeneous isothermal wall boundary conditions. The outlet boundary on the right was maintained at zero flux across the boundary, while at the inlet a velocity profile of $\sin (\pi y)$ was specified. The exact solution for this case can be given by

$$
\left(\frac{e^{R_{1} x} R_{2} e^{R_{2} L}-e^{R_{2} x} R_{1} e^{R_{1} L}}{R_{2} e^{R_{2} L}-R_{1} e^{R_{1} L}}\right) \sin (\pi y)
$$




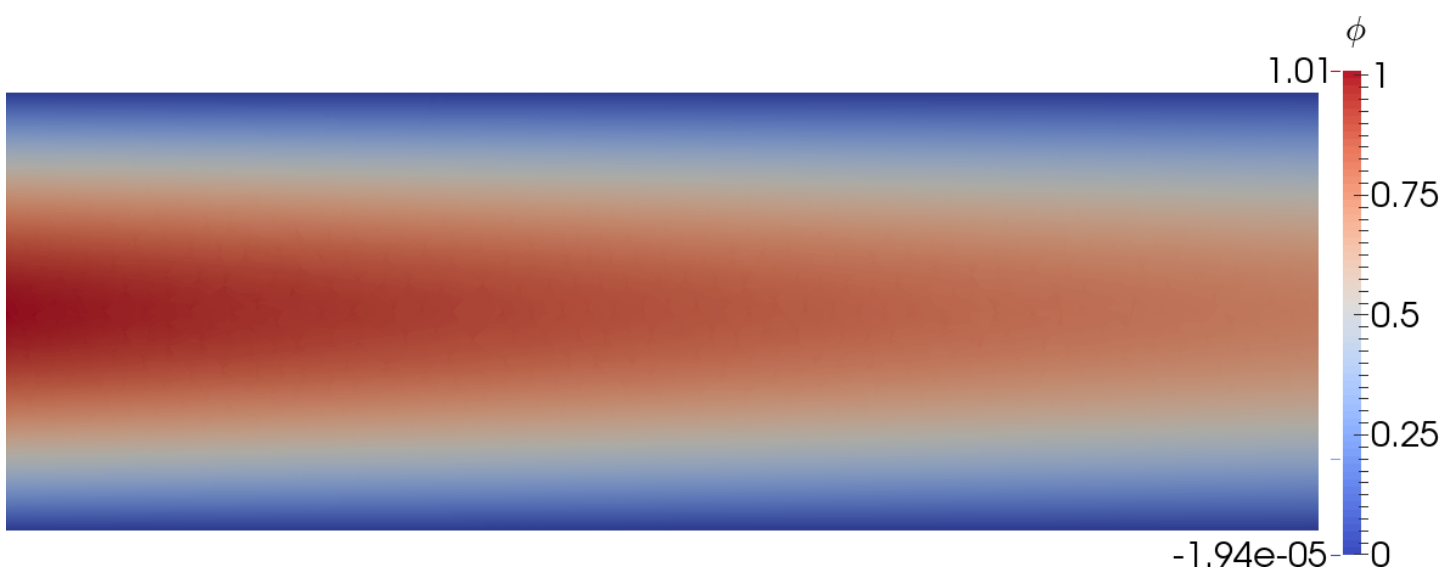

Figure 5.8: Advection-diffusion solution $(\alpha=0.01)$

where

$$
R_{1}=\frac{H}{2 \alpha}+\sqrt{\frac{H^{2}}{4 \alpha^{2}}+\pi^{2}} ; R_{2}=\frac{H}{2 \alpha}-\sqrt{\frac{H^{2}}{4 \alpha^{2}}+\pi^{2}} .
$$

The final solution is shown in Figure 5.8 with the coefficient of diffusion $(\alpha)$ set equal to 0.01 on an unstructured mesh consisting of 2162 control volumes. This value of 0.01 for the coefficient of diffusion causes the flow to be advection dominated while diffusion dominance is achieved with a value of at least 1 .

The orders of accuracy of the advection-diffusion case, flux and flux integral have been plotted and compared in Figure 5.9. The tests were run on 5 successively refined meshes containing 138, 530, 2162, 8655 and 35156 isotropic triangles.

The flux integral obtained using the initial data has been shown in Figure 5.9a. It shows good overall improvement with respect to a second-order least squares scheme. The diffusion dominant case reflects better order of accuracy while even the advection dominant case shows accuracy gains by a constant factor of two.

The solution convergence in Figure 5.9b, shows that using $\mathrm{H}_{1}$ discretization for the diffusive fluxes provides significant gains in solution accuracy by at least a factor of two, even with an advection dominant flow. In both the advection and the diffusion dominant cases, $\mathrm{H}_{1}$ reconstruction performs at a balance between second and third order least-squares schemes.

Table 5.2 lists the order of accuracy achieved in both advection and diffusion dominant cases for each scheme. Once again the rates of convergence have been averaged over the 


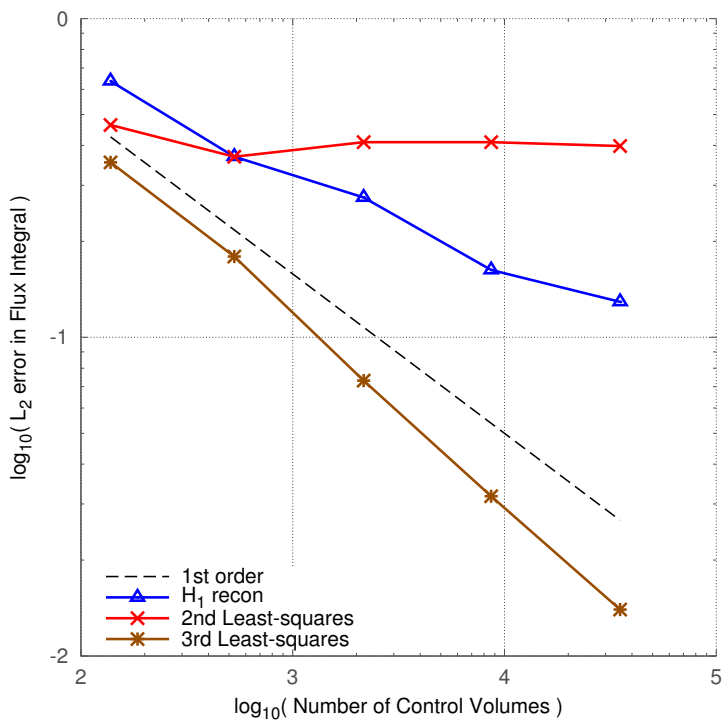

Diffusion Dominant $(\alpha=1)$

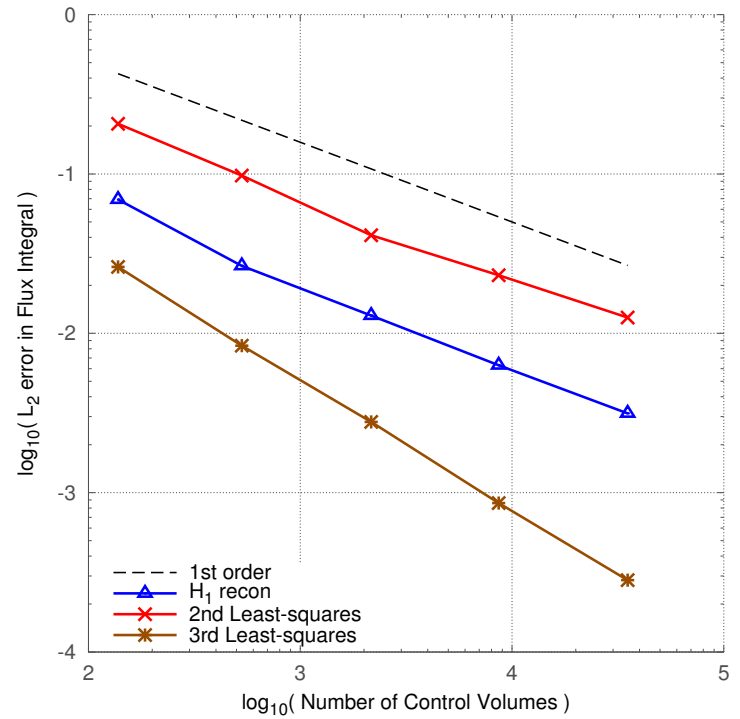

Advection Dominant $(\alpha=0.01)$

(a) Flux-integral convergence rates (initial data)

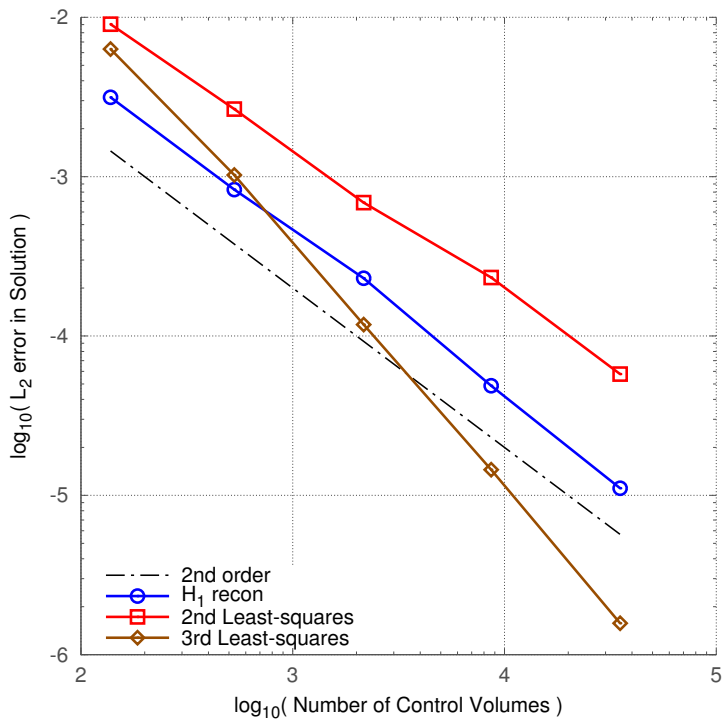

Diffusion Dominant $(\alpha=1)$

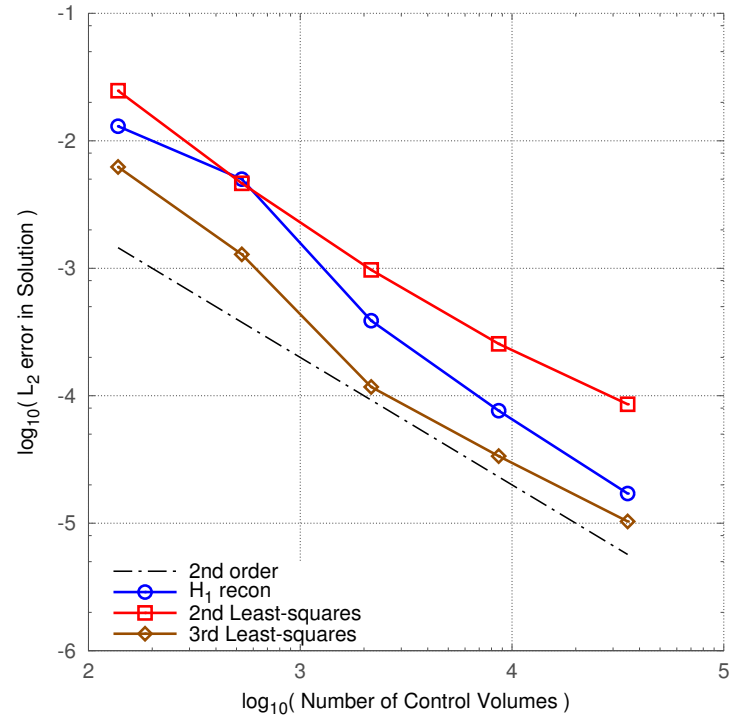

Advection Dominant $(\alpha=0.01)$

(b) Solution convergence rates

Figure 5.9: Advection-Diffusion 2D test results 
five refinement stages.

\begin{tabular}{|l|c|c|c|c|}
\hline \multicolumn{2}{|c|}{} & $\begin{array}{c}\text { Second-order } \\
\text { Least Squares }\end{array}$ & $\begin{array}{c}\mathrm{H}_{1} \\
\text { Reconstruction }\end{array}$ & $\begin{array}{c}\text { Third-order } \\
\text { Least Squares }\end{array}$ \\
\hline \hline \multirow{2}{*}{$\begin{array}{l}\text { Diffusion } \\
\text { Dominant }\end{array}$} & $\begin{array}{c}\text { Flux integral } \\
\text { (initial data) }\end{array}$ & 0.06 & 0.51 & 1.17 \\
\cline { 2 - 5 } & Solution & 1.83 & 2.01 & 3.01 \\
\hline $\begin{array}{l}\text { Advection } \\
\text { Dominant }\end{array}$ & $\begin{array}{c}\text { Flux integral } \\
\text { (initial data) }\end{array}$ & 1.01 & 1.15 & 1.63 \\
\cline { 2 - 5 } & Solution & 2.08 & 2.31 & 2.50 \\
\hline
\end{tabular}

Table 5.2: Orders of accuracy for advection-diffusion problem

Overall apart from the accuracy gains, the presence of the upwind convective flux also greatly enhances the stability of the $\mathrm{H}_{1}$ discretization, almost playing the part of the jump term itself. A more in-depth discussion about stability of the scheme will be presented in Section 6.1. The coefficient of diffusion has also been observed to affect the added stability brought in by the upwind discretization, being largest in the advection dominant situation. 


\section{Chapter 6}

\section{Characterization Tests and}

\section{Behavioural Analysis}

Apart from pure numerical accuracy, there are other desirable qualities that one would expect from a numerical scheme. The foremost among these is the issue of stability and robustness which we will look at first. We will also analyze the truncation error that results from our scheme followed by cost comparison studies. A further opportunity to study the behaviour of $\mathrm{H}_{1}$ reconstruction arose when the ANSYS Fluent software development team expressed their interest in our new scheme. They kindly offered to provide an insight into the flux discretization scheme used in the Fluent software. This enabled us to perform detailed comparative studies with their discretization scheme as well as possible avenues of implementing the $\mathrm{H}_{1}$ finite-volume scheme into their discretization. Our findings from this study have been presented in the last section.

\subsection{Stability Analysis}

In this section, we analyze the stability of the various schemes discussed. Stability analysis is crucial since it provides valuable insights into the robustness of the scheme on the variety of complex meshes that are encountered in real world applications.

In 2012, Ollivier-Gooch and Roy [42] proposed an eigenvalue analysis tool for unstructured finite-volume schemes. This tool, in addition to its original purpose of analyzing the asymptotic order of the truncation error, can also be used to study the stability of a scheme through the analysis of the eigenvalues of its Jacobian matrix.

The location of the eigenvalues of a space discretization scheme is indicative of the stability of the discretization scheme [27]. Specifically, the stability of the system of ODE's 
obtained from the method of lines is guaranteed if and only if all its eigenvalues have negative real components.

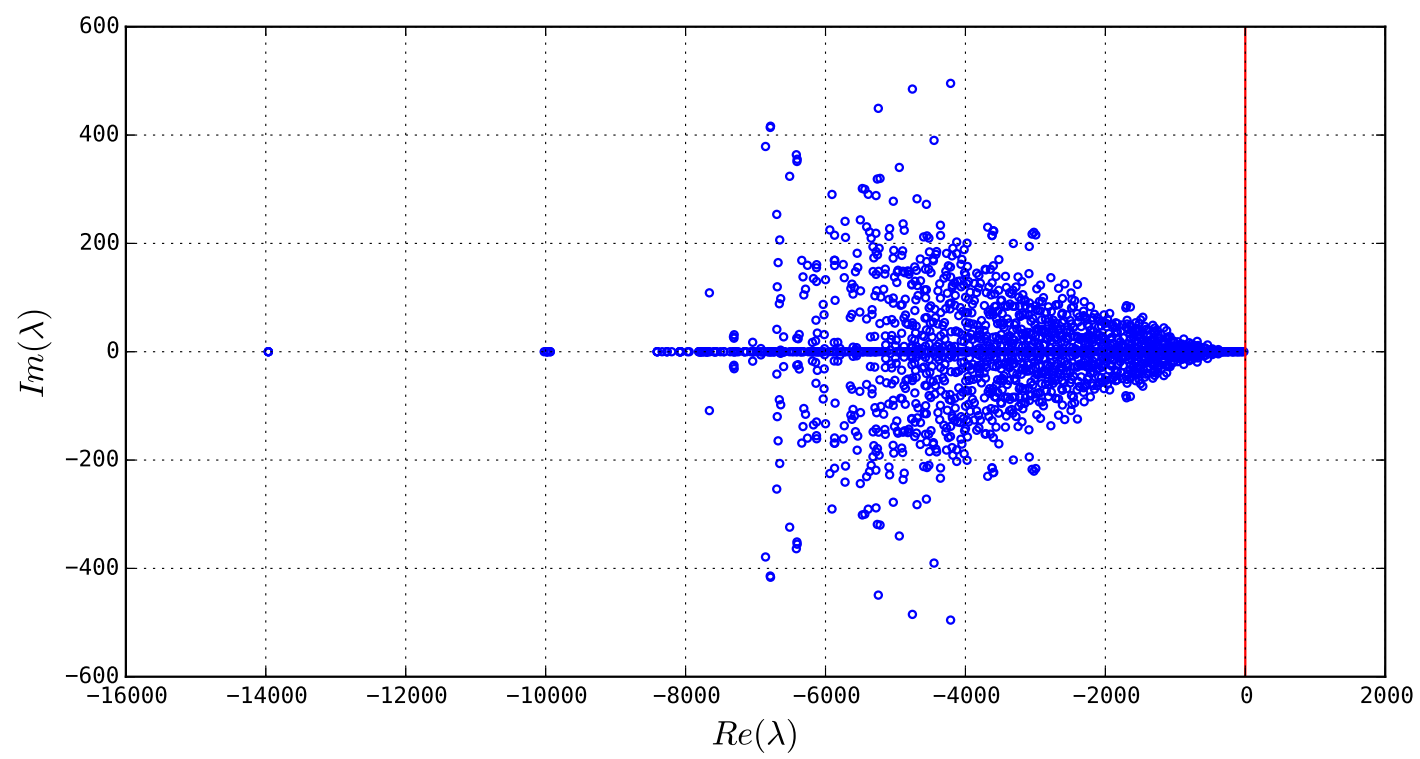

(a) Second-order least squares reconstruction with centroids

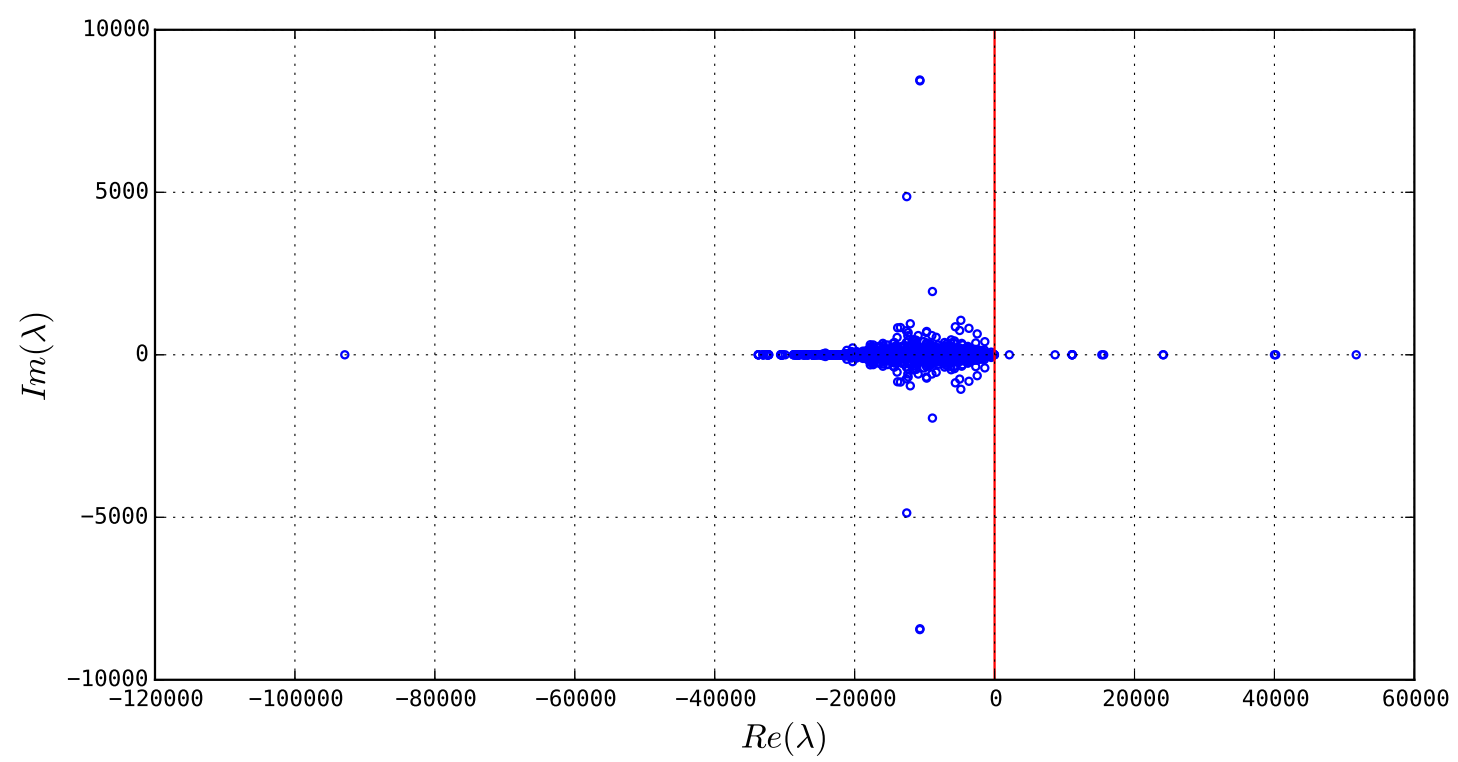

(b) Second-order least squares reconstruction with circumcentres

Figure 6.1: Eigenvalue plots - centroid vs. circumcentres

For instance, the eigenvalue spectrum of a regular second-order least squares reconstruction scheme with respect to its Jacobian matrix, as shown in Figure 6.1a, has all well-behaved eigenvalues with negative real components and consequently, this is a stable 
problem. Although the eigenvalues of the Poisson equation should be pure real, that is not the case here. This can be attributed to the unstructured nature of the underlying mesh that our Jacobian matrix is based upon and as a result it is non-symmetric. Thus the lack of symmetry contributes to the imaginary components of the eigenvalues. This and most of the other tests that follow were run on a cell-centred unstructured mesh for the Poisson model problem, unless specified otherwise.

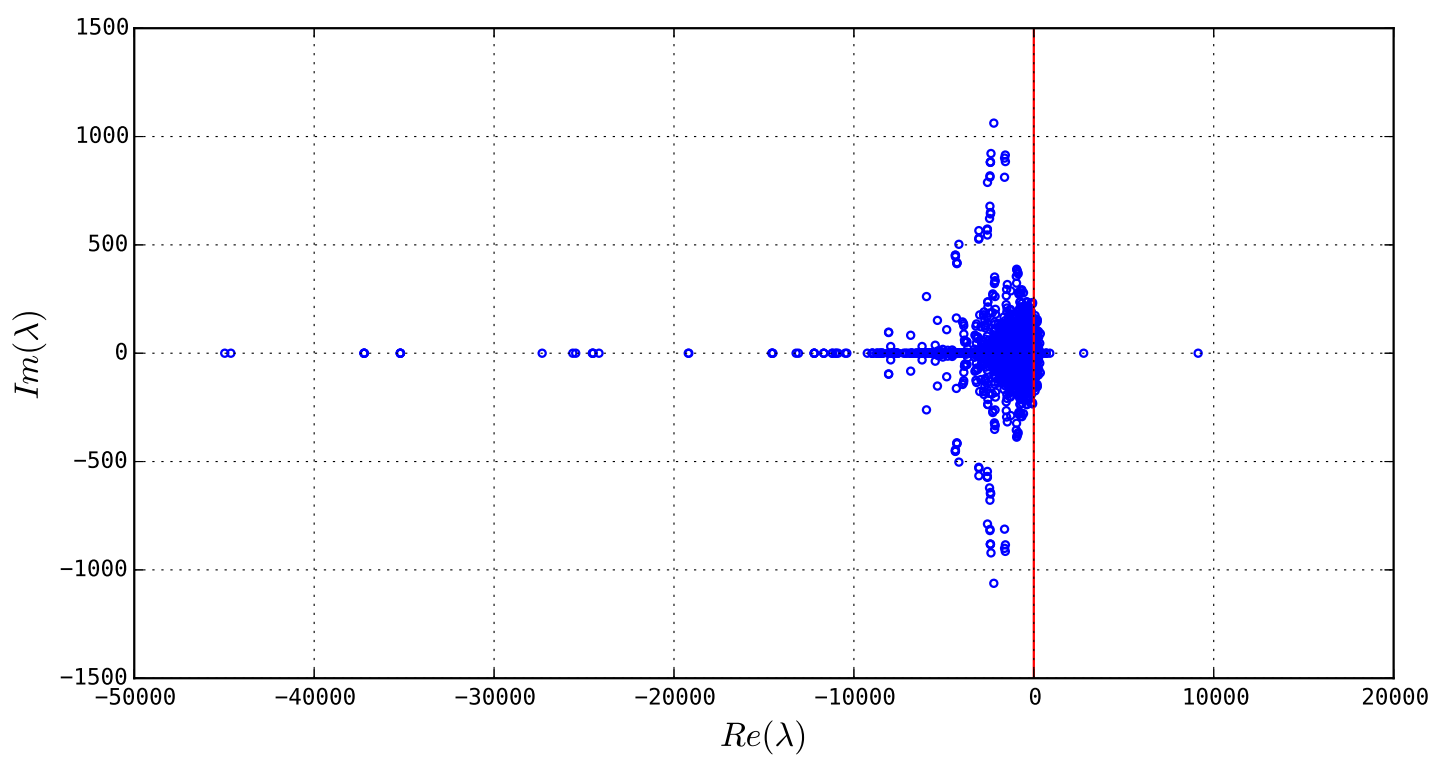

(a) $\mathrm{H}_{1}$ reconstruction with circumcentres

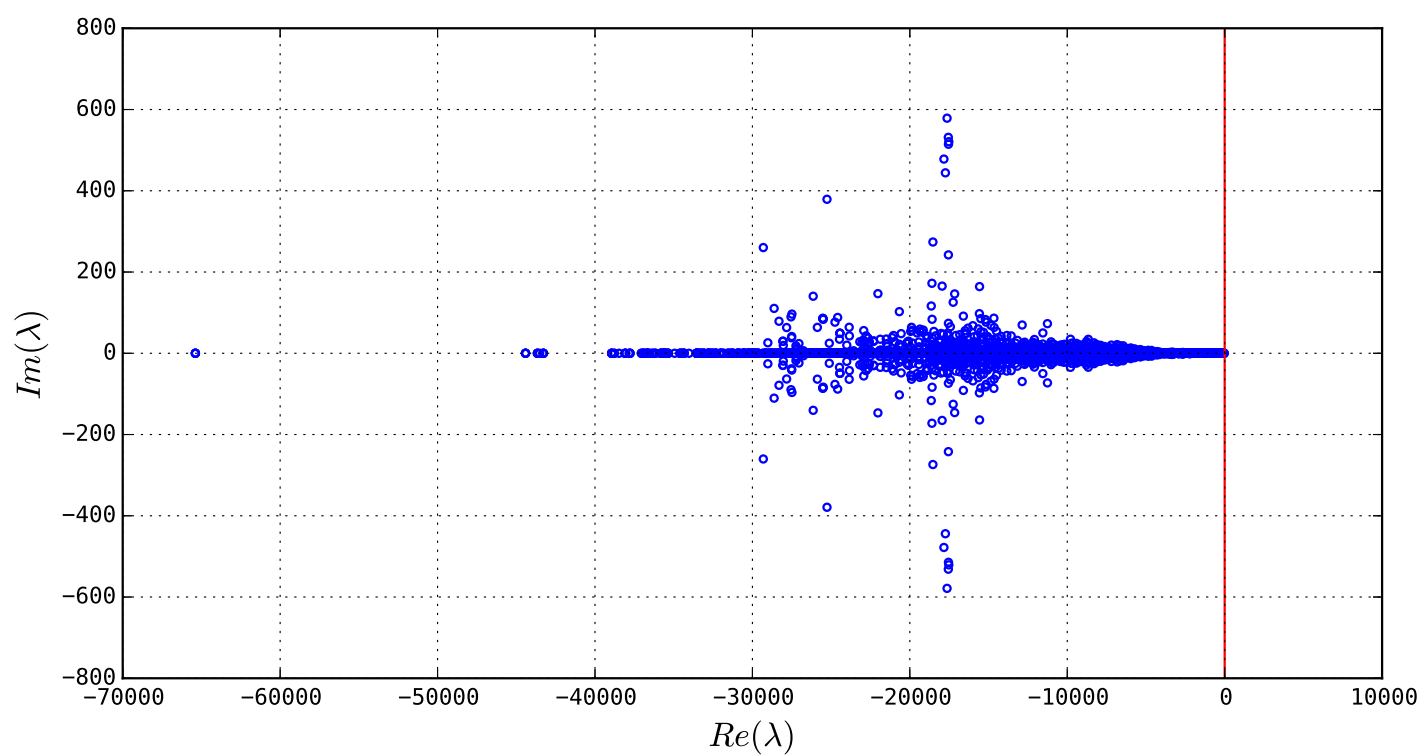

(b) $\mathrm{H}_{1}$ reconstruction with containment centres, high accuracy jump term

Figure 6.2: $\mathrm{H}_{1}$ reconstruction eigenvalue plots 


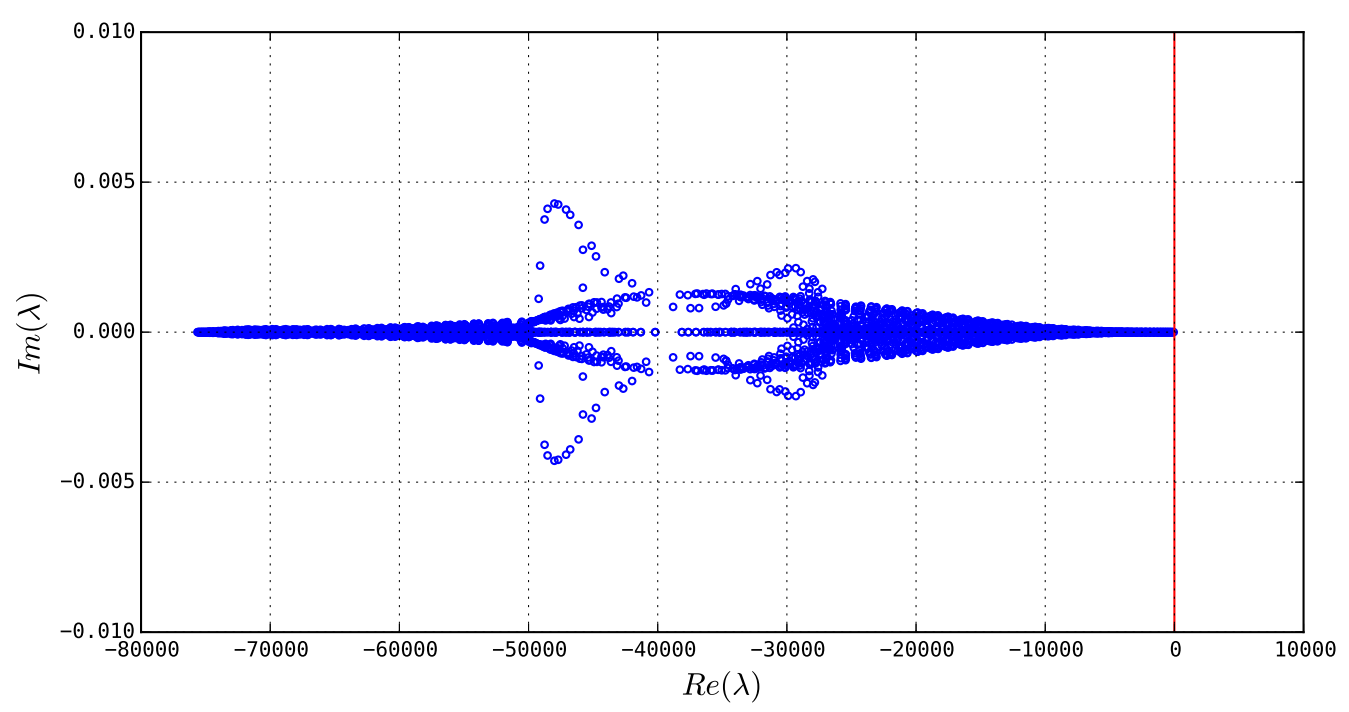

Figure 6.3: $\mathrm{H}_{1}$ reconstruction on equilateral mesh

In contrast Figure $6.1 \mathrm{~b}$ illustrates the eigenvalue spectrum of the same second-order least squares reconstruction but with circumcentres as the control-volume reference points instead of the traditional centroids. We can instantly observe a major degradation of the eigenvalue spectrum, with seven of the eigenvalues lying on the positive real half of the plane (the unstable half), which provides visual proof of the instability of the scheme involving circumcentres. Unfortunately $\mathrm{H}_{1}$ reconstruction on a cell-centred unstructured mesh with circumcentres is also unstable as shown by the positive eigenvalues in Figure 6.2a.

We were successful in enhancing the stability of the $\mathrm{H}_{1}$ reconstruction scheme with the use of containment centres instead of circumcentres as control-volume reference points and the inclusion of a high-accuracy jump term. The resulting eigenvalues are plotted in Figure 6.2b. As can be seen, they are all well behaved and lie on the stable half of the complex plane. Likewise the eigenvalue spectrum of $\mathrm{H}_{1}$ reconstruction is stable on an equilateral (structured) mesh, as seen in Figure 6.3.

Figure $6.4 \mathrm{a}$ shows the eigenvalue spectrum of the stable second-order least squares scheme on the advection-diffusion equation with a coefficient of diffusion set to 0.01. The stabilizing influence of the upwind discretization of the convective terms is clear when we look at the well behaved eigenvalues of $\mathrm{H}_{1}$ reconstruction with only circumcentres and without any jump term, in Figure 6.4b. 


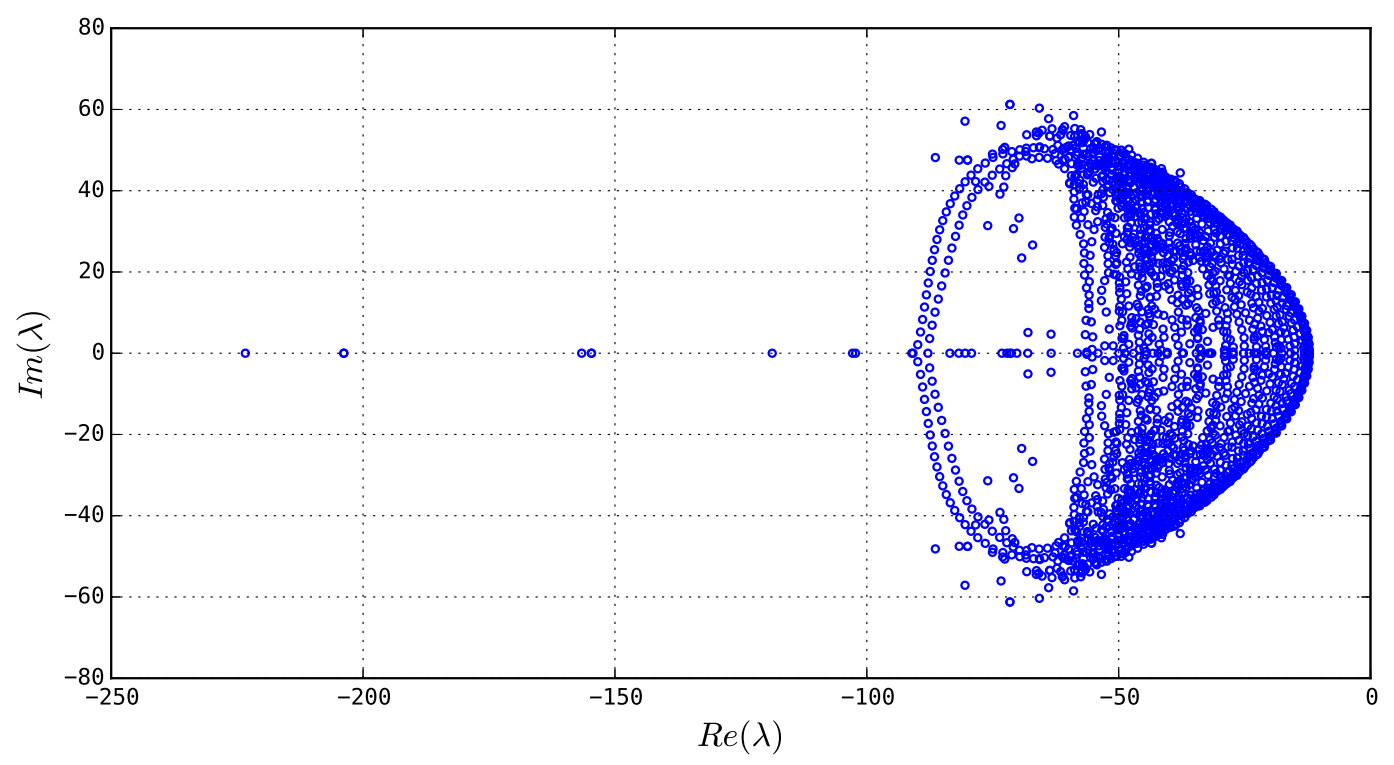

(a) Second-order least squares with centroids (advection-diffusion)

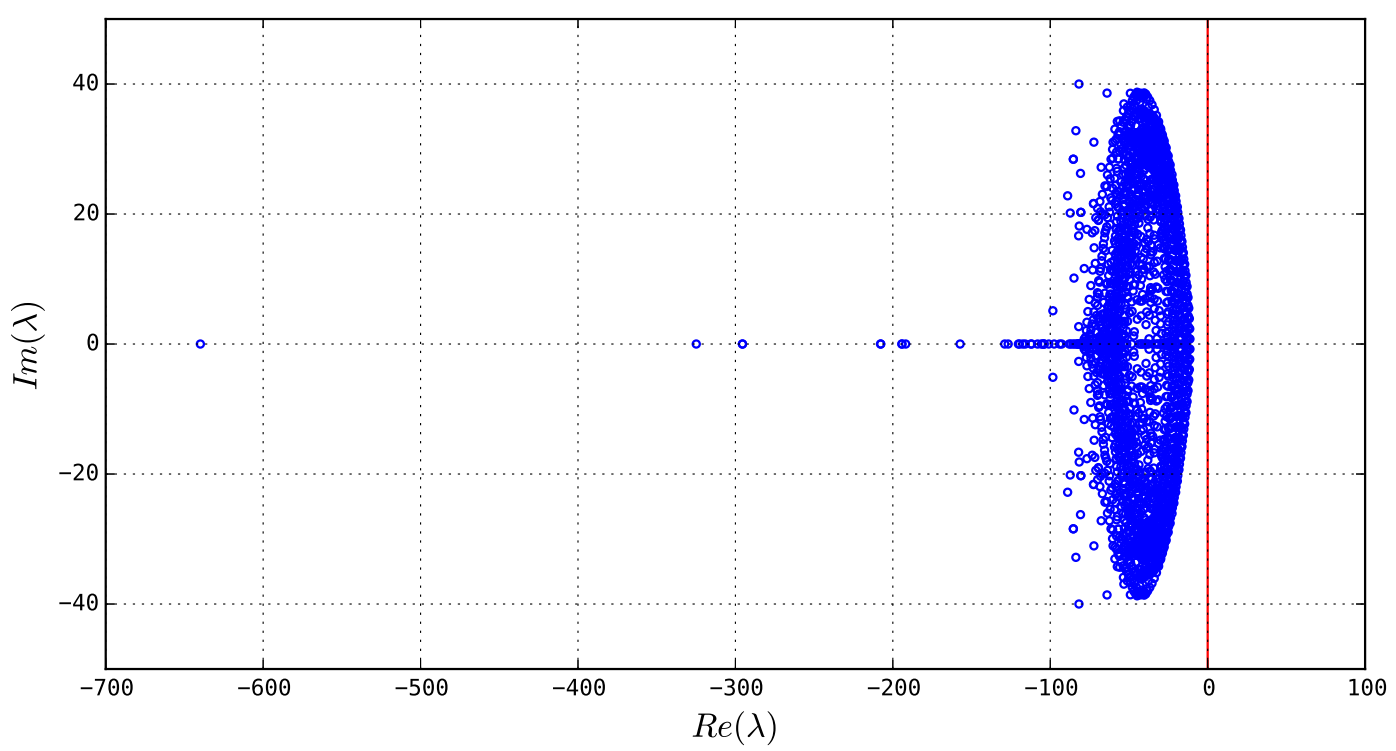

(b) $\mathrm{H}_{1}$ reconstruction with circumcentres (advection-diffusion)

Figure 6.4: Advention-diffusion eigenvalue plots

\subsection{Truncation Error Analysis}

The truncation error, as discussed earlier, is the error in approximating the continuous PDEs with the finite discretized equations. It has recently emerged as another measure of accuracy of the scheme and its estimates find varied applications such as improving the solution through defect correction, finding the error in an engineering output quantity via 
adjoint problems [13], mesh adaptation [48], as well as estimation of discretization errors via the error transport equation [49]. Ollivier-Gooch and Van Altena [43] performed truncation error analysis by analyzing the Taylor series of the Laplacian on structured triangular meshes. Their work was extended to arbitrary meshes and any linear PDE by Jalali and Ollivier-Gooch [20]. The result of their analysis is a set of Taylor series coefficients for the truncation error obtained for every control volume, which when combined with the local solution derivatives gives the truncation error itself. The same technique is herein used to evaluate and analyze how the $\mathrm{H}_{1}$ finite-volume scheme fares in comparison to the other two regular least-squares schemes. The truncation error magnitudes were found for the Poisson model problem on an unstructured mesh in a square domain.

The truncation error is known to be identical to the flux integral or the residual obtained from smooth initial data. Running the exact solution data through the discretization scheme once provides the finite discrete estimate of the flux integral. This estimate can then be compared to the exact known flux integral to obtain the exact truncation error for that scheme. Hence the asymptotic convergence rate of truncation error is the same as that for the flux integral with exact solution data. The flux integral convergence plot for Poisson has been presented again in Figure 6.5 for convenience. As with the flux integral, the asymptotic convergence rate of truncation error in least-squares schemes is one order lower than the gradient and hence two orders lower than the solution. Thus as can be seen in Figure 6.5, the truncation error for second-order least squares is zero order, while that of third-order least squares is first order. The $\mathrm{H}_{1}$ finite-volume scheme as we have already seen in section 5.2 performs on par with third-order least squares asymptotically.

Figure 6.6 juxtaposes the truncation errors from each of the three schemes being analyzed. The first row shows the truncation error, while the second row plots the magnitude of truncation error against the real part of the corresponding eigenvalues. A logarithmic scale has been used for both axes in order to enhance the visualization; however taking into consideration that the real part of the eigenvalues $\operatorname{Re}(\lambda)$ is negative (for our stable scheme), $-\operatorname{Re}(\lambda)$ is plotted instead. Plotting in such a manner is much more insightful than just looking at the convergence rates since it provides an explanation for the behaviour of the convergence [21]. It provides insights into the distribution of the error with respect to the 


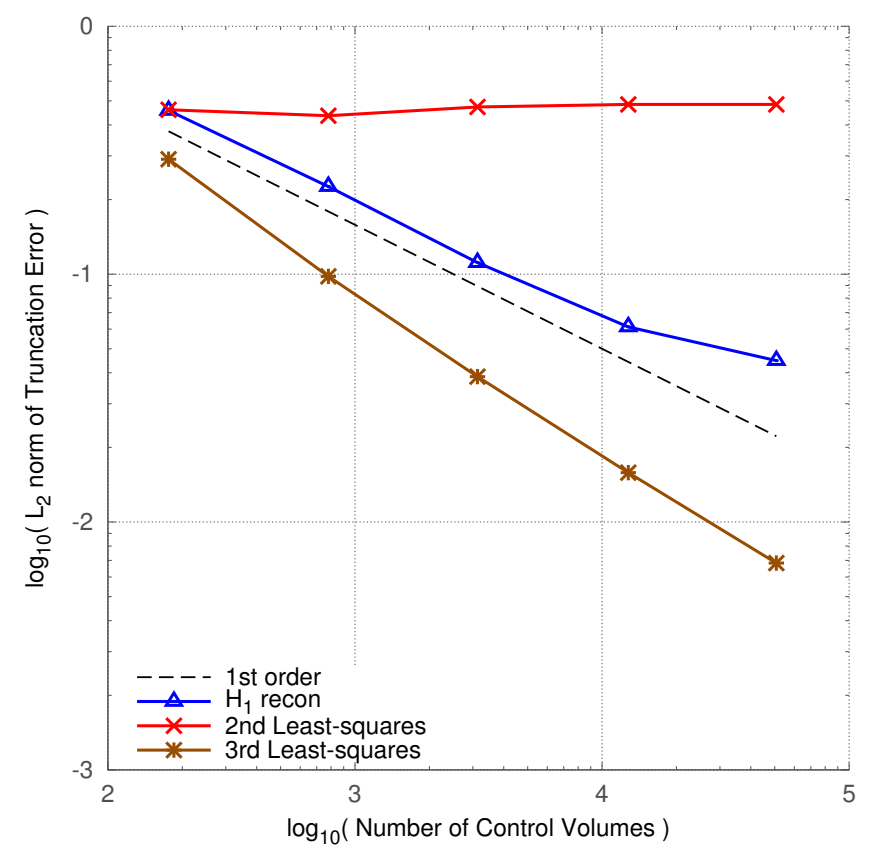

Figure 6.5: Truncation error convergence rate

high and low frequency modes of the eigenvalues. The leftmost eigenvalues correspond to the high frequency modes while the rightmost ones are the smoother low frequency errors. It is a general trend for the high frequency errors to dominate the eigendecomposition of the truncation error as evidenced by the truncation error magnitudes of least-squares schemes in Figure 6.6(a) and (c). For instance, if we take a careful look at the plot for the third order scheme we can observe that the majority of the eigenvalues are on the high-frequency band to the left and those dominate the truncation error as well. Those high-frequency modes that occupy the left half of the spectrum correspond to the boundary regions, while the right-most low frequency modes correspond to interior regions. The low frequency eigenvalues have considerably smaller truncation error magnitude. Not surprisingly, errors are larger for the boundary regions.

However, $\mathrm{H}_{1}$ reconstruction seems to defy this trend to some extent, with the high as well as the low frequency modes being of almost similar magnitudes. Yet in overall magnitude it is still only comparable to second-order least squares scheme. The smoother modes unfortunately seem to have suffered in $\mathrm{H}_{1}$ reconstruction and have higher error. There also exist certain outlier high-error eigenvalues which we believe result from the not-so-accurate 


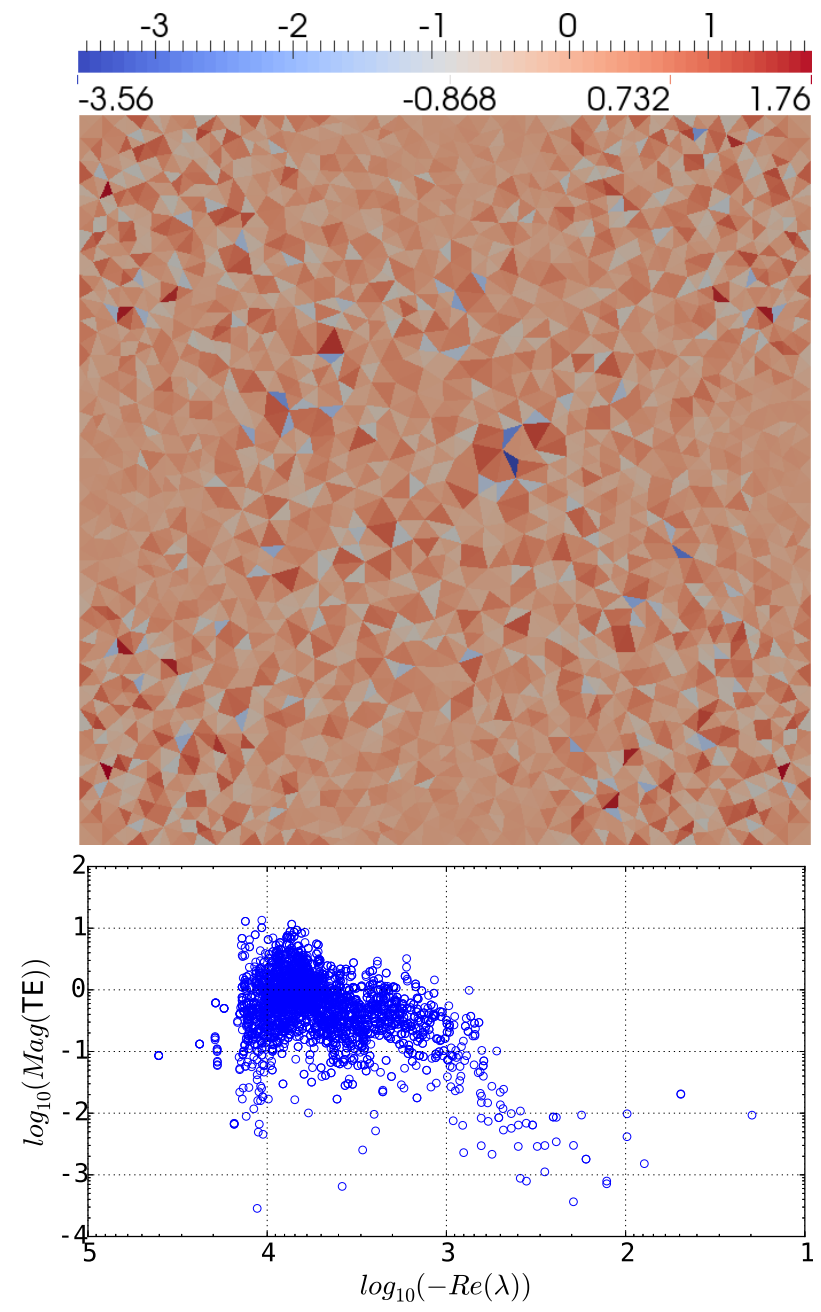

(a) Second-order least squares
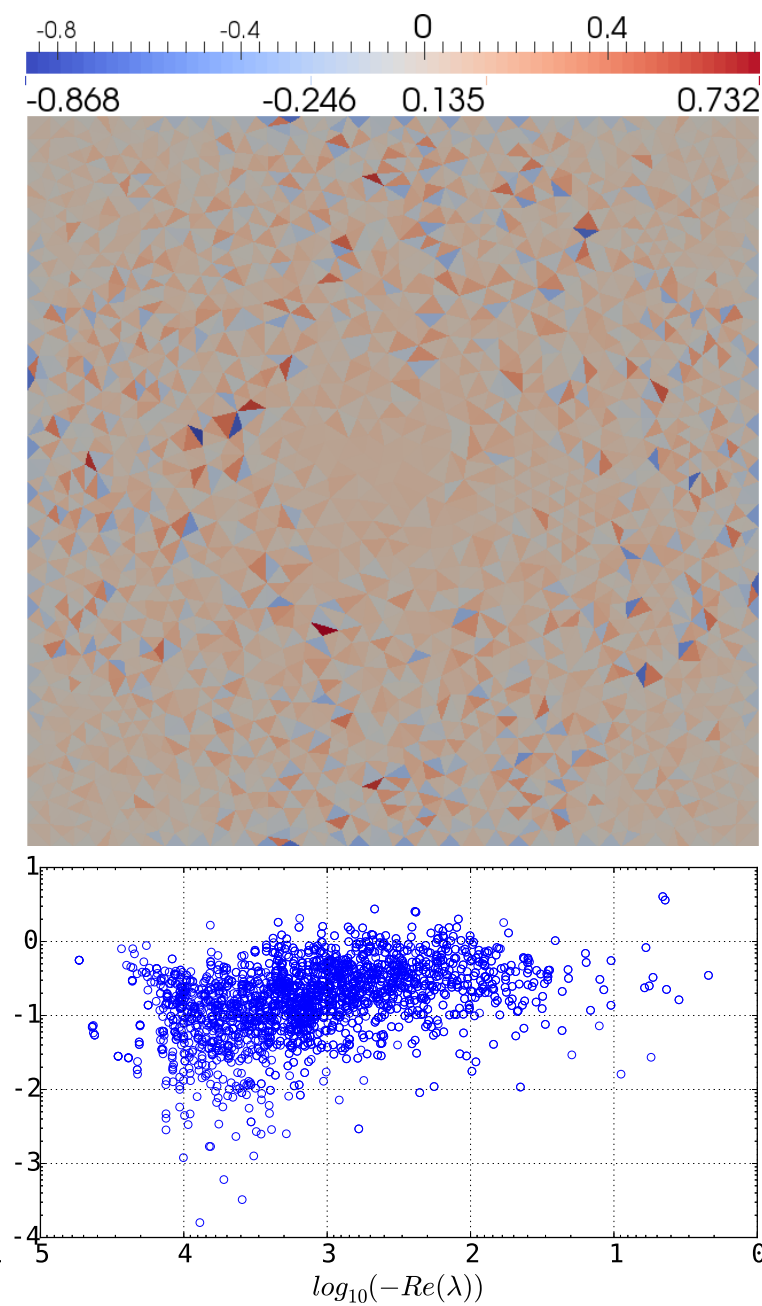

(b) $\mathrm{H}_{1}$ finite volume

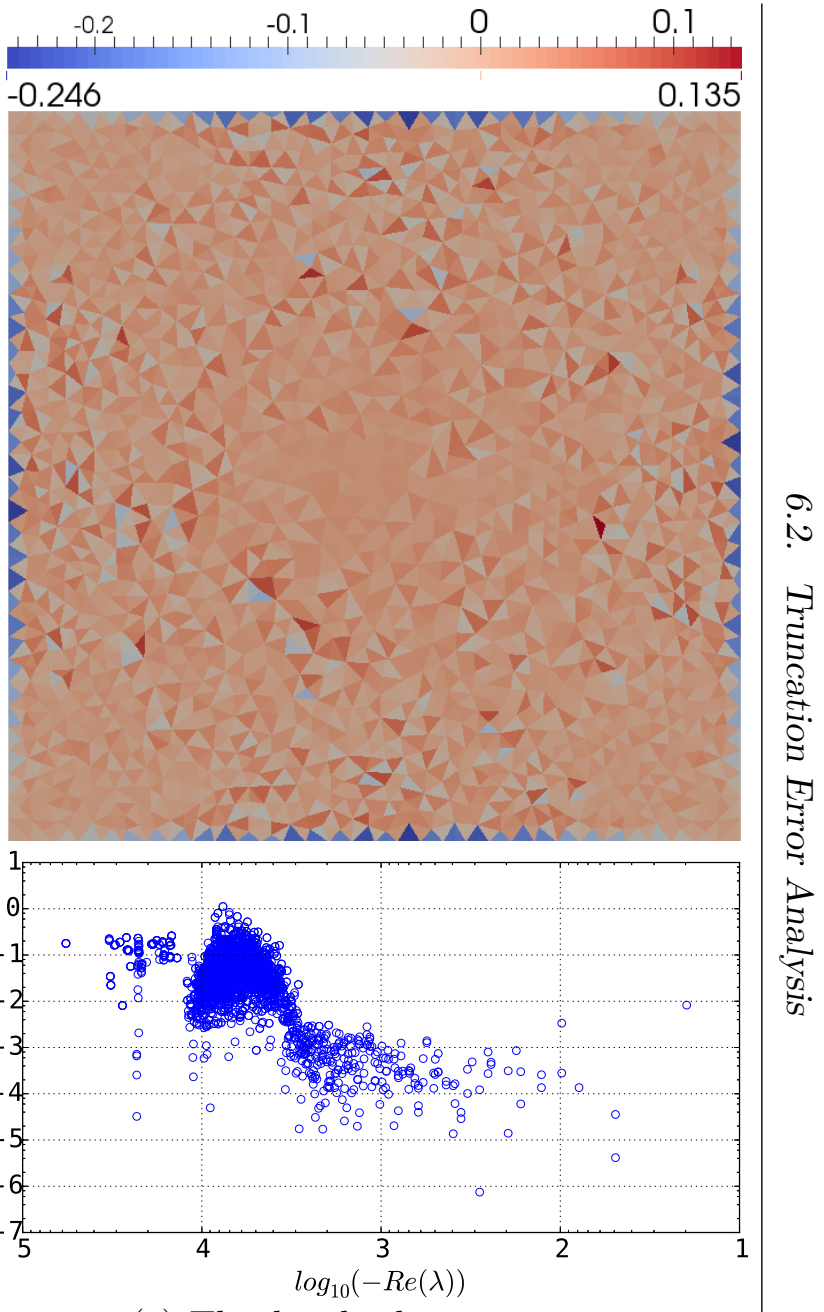

(c) Third-order least squares 


\begin{tabular}{|c|c|c|c|c|c|c|}
\hline \multirow{2}{*}{$\begin{array}{c}\text { Number } \\
\text { of Cells }\end{array}$} & \multicolumn{2}{|c|}{$\begin{array}{c}\text { Convergence Wall Time per } \\
\text { Iteration (seconds) }\end{array}$} & \multicolumn{3}{|c|}{$\begin{array}{c}\text { Jacobian Assembly Time } \\
\text { (seconds) }\end{array}$} \\
\cline { 2 - 7 } & 2 ndLS & $\mathrm{H}_{1}$ & $3 \mathrm{rdLS}$ & 2 ndLS & $\mathrm{H}_{1}$ & 3 rdLS \\
\hline \hline 176 & $1.08 \times 10^{-2}$ & $1.30 \times 10^{-2}$ & $2.17 \times 10^{-2}$ & $3.86 \times 10^{-3}$ & $5.21 \times 10^{-3}$ & $1.09 \times 10^{-2}$ \\
\hline 3132 & $1.45 \times 10^{-1}$ & $1.84 \times 10^{-1}$ & $3.40 \times 10^{-1}$ & $6.74 \times 10^{-2}$ & $9.19 \times 10^{-2}$ & $1.88 \times 10^{-1}$ \\
\hline 50715 & 2.52 & 3.23 & 5.66 & 1.09 & 1.50 & 3.01 \\
\hline
\end{tabular}

Table 6.1: Wall times

containment centres.

Moreover, if we look at the truncation error visualizations, on the top row of Figure 6.6, then the $\mathrm{H}_{1}$ reconstruction seems to more closely resemble the third order discretization in terms of the underlying smoothness but the extreme values are almost four times that of third-order least squares reconstruction. Meanwhile, the second-order scheme extremes are almost a similar factor larger than $\mathrm{H}_{1}$ reconstruction.

\subsection{Cost Comparisons}

Next, we compare the computational cost incurred with the $\mathrm{H}_{1}$ finite-volume scheme against the conventional least-squares schemes on the Poisson model problem.

Let us begin by analyzing the Jacobian calculations and its memory requirements. As indicated by Figure 6.7, although the memory required to store the Jacobian matrix is the same, the total Jacobian matrix assembly time of the new scheme is much lower than a full third-order least-squares scheme. The Jacobian memory allocation process accounts only for the size of the reconstruction stencil used and since $\mathrm{H}_{1}$ reconstruction utilizes the same nine cell stencil as the third-order scheme, we would expect their memory requirements to be identical. However, the $\mathrm{H}_{1}$ scheme uses just one Gauss quadrature point while the thirdorder least-squares scheme uses two. This translates to savings in the Jacobian assembly time, making it twice as fast as third-order and only slightly slower than the second-order Jacobian assembly time. This relation is clear when we look at the Jacobian assembly times listed in Table 6.1.

Moreover, our modifications detailed in section 3.1 are reflected in the convergence wall 


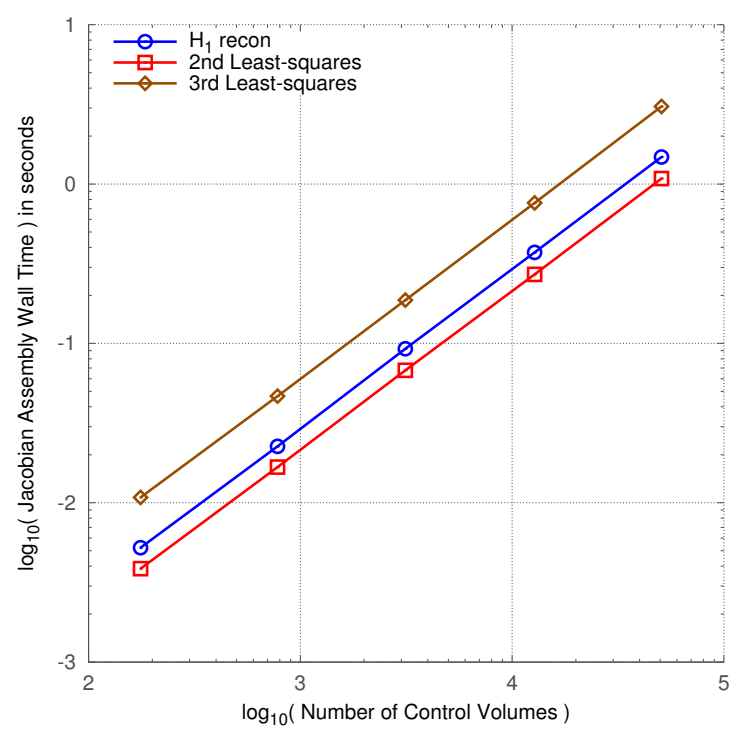

(a) Jacobian calculation wall times

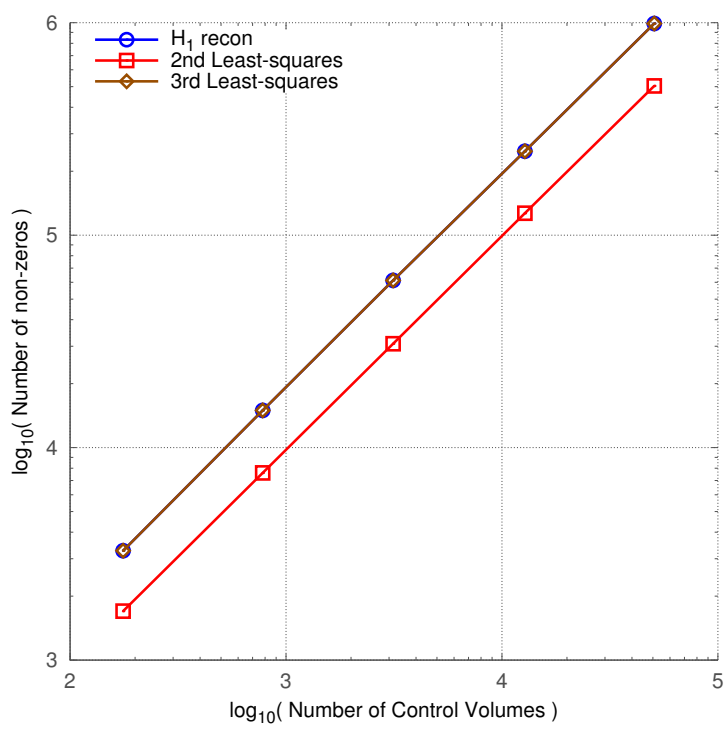

(b) Jacobian memory usage

Figure 6.7: Jacobian calculation comparisons

time per iteration of the new scheme which is also lower than a third-order least-squares scheme as seen in Figure 6.8b. Table 6.1 also lists the convergence wall time per iteration for each scheme. Unfortunately the $\mathrm{H}_{1}$ scheme currently takes a few additional iterations (pseudo-time steps) to reach full convergence which causes the total convergence time to be larger.

All the more insightful is the Figure 6.9 where we can see that for any given accuracy of the flux accuracy that we may require (say $10^{-3}$ ) then the quickest way to achieve that is by using the third-order least-squares scheme followed closely by our $\mathrm{H}_{1}$ scheme and then, by further off, the second-order least-squares scheme. We believe that by rectifying the cause of the few additional iterations that $\mathrm{H}_{1}$ scheme requires, we can manage to improve this plot, by becoming comparable to, if not better than the third-order least-squares scheme. Figure 6.9 also portrays the reason why high-order methods are generally more preferable to low-order schemes.

The impact of the savings in Jacobian assembly time is evident when we take a look at the time profile analysis. The Jacobian evaluation clearly dominates the convergence wall time as seen in Figure 6.10. The pie chart diameters for each scheme is proportional to its convergence wall time per iteration. The other prominent processes that make up the rest 
of the solver run time include the function evaluation and the KSP solve. The former is the flux integration process, which includes the evaluation of the fluxes. The KSP solve is linear solver part of our time-advance algorithm, solving the linear system that is obtained at each time step. The Generalized Minimal Residual (GMRES) algorithm is the Krylov subspace iterative linear solver that is used in ANSLib [32].

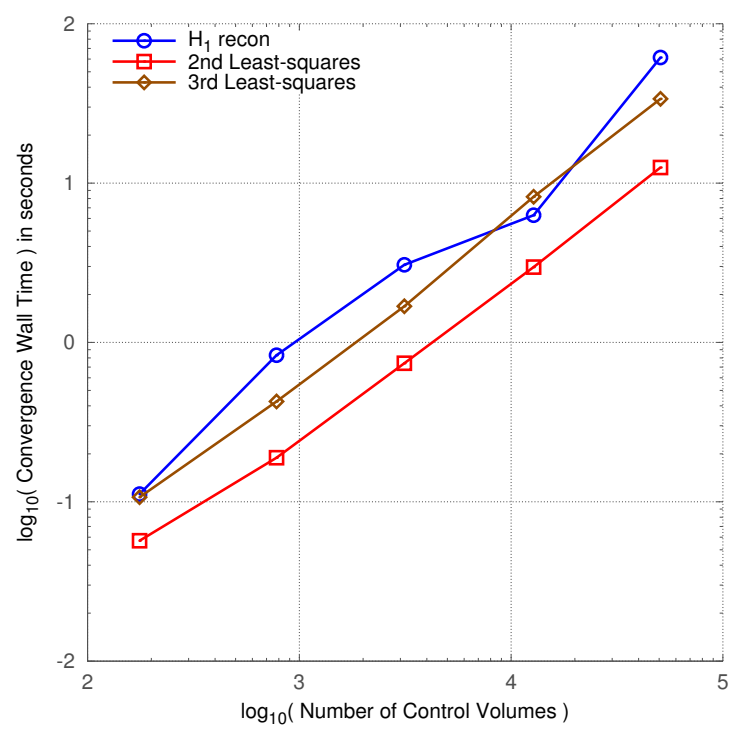

(a) Total convergence time

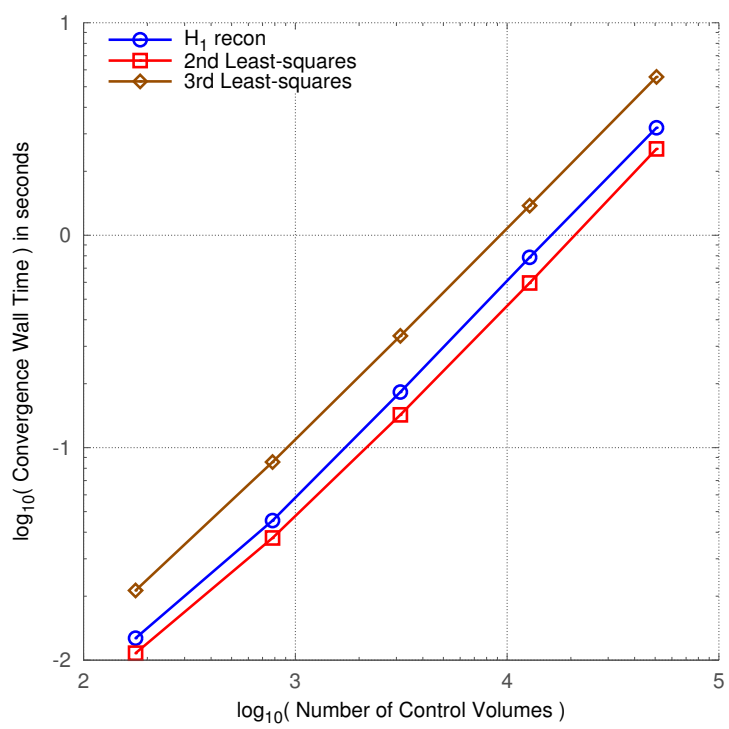

(b) Convergence time per iteration

Figure 6.8: Comparison of total wall clock time required for convergence

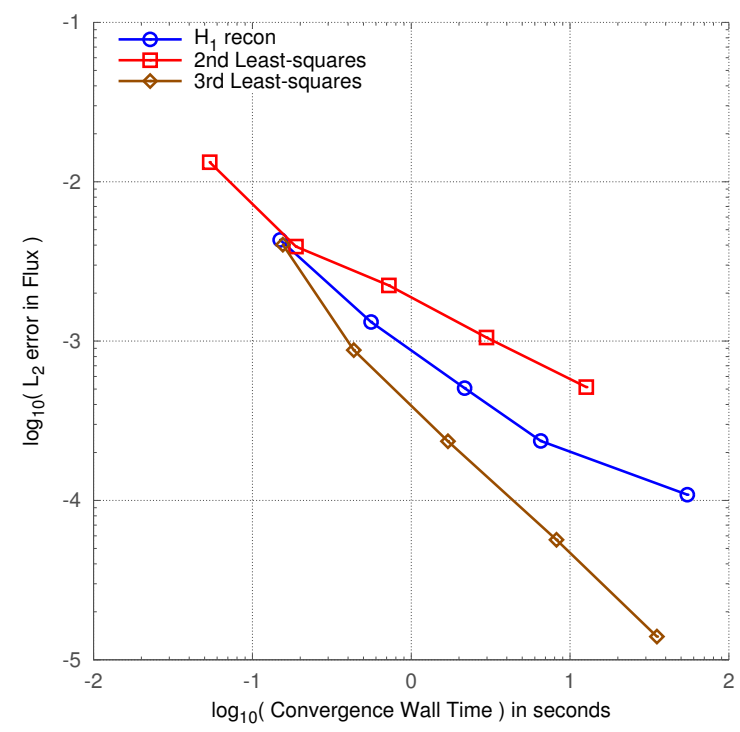

Figure 6.9: Flux error (steady state) vs total wall clock time required for convergence 

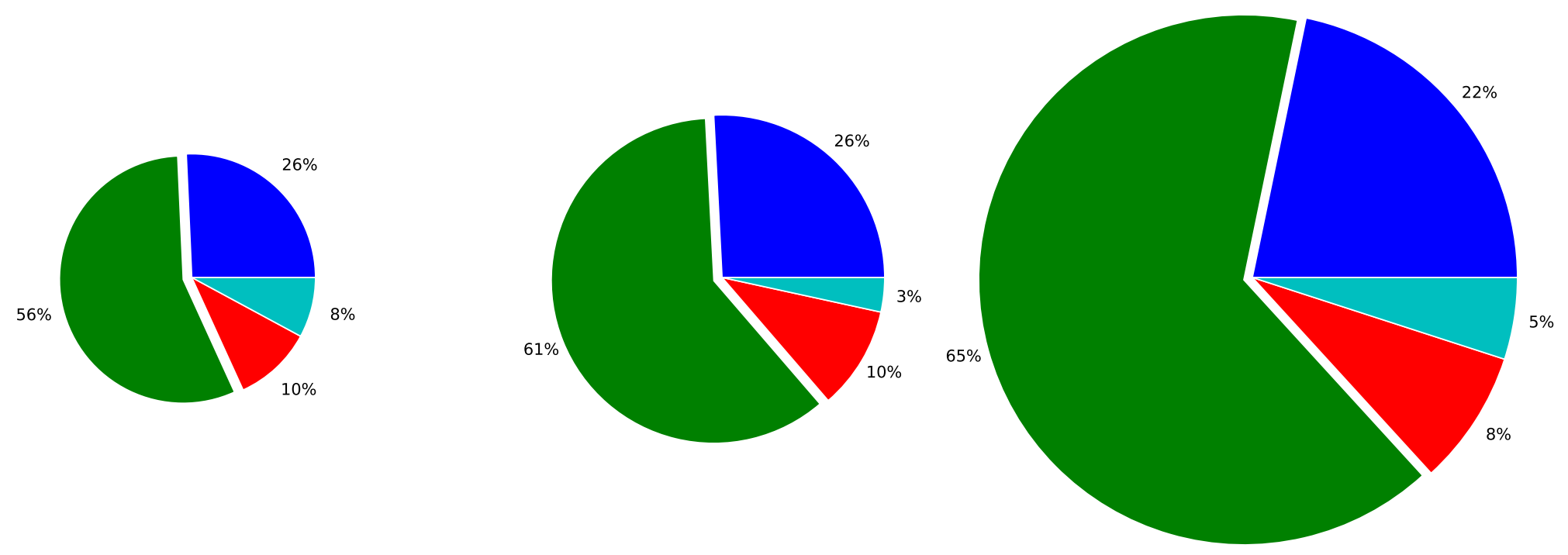

Figure 6.10: Time profile analysis 


\subsection{Behavioural Analysis of the $\mathrm{H}_{1}$ Finite-Volume Scheme in the ANSYS Fluent Discretization}

The interest expressed by the numerics software development team of ANSYS Fluent was an invaluable opportunity to further study the behaviour of the $\mathrm{H}_{1}$ finite-volume scheme when implemented within a different flux discretization framework. It also provided an opportunity to baseline our ANSLib numerical simulation software with a well established commercial software like Fluent. We begin with a brief description of the flux discretization scheme used in the Fluent software, followed by our approach to implement the $\mathrm{H}_{1}$ finite-volume scheme within their discretization framework. We will then look at how our implementation of the Fluent diffusion discretization scheme within ANSLib matches with the results from the Fluent software itself. Once satisfied with our baseline calibrations, we will look at how our modifications fare in comparison with other schemes.

\subsubsection{Fluent Diffusive Flux Discretization}

The Fluent flux discretization is based on the scheme of Mathur and Murthy [28], with additional improvements over the years. Foremost among the modifications is the inclusion of a second-order least-squares solution reconstruction which is very similar to the one used in ANSLib solver.

A central feature of the diffusive flux scheme implemented in Fluent is the "two-pass" gradient computation, which evaluates two distinct forms of gradients. These two gradient computations are discussed below followed by the final diffusive flux formulation and a discussion about how it is handled at the boundaries.

\subsubsection{Reconstruction Gradients}

The first pass performs a second-order least-squares solution reconstruction, which provides base gradients that are only first-order accurate, as anticipated from our discussions in Chapter 4. This reconstruction uses the first layer of face-neighbours to form the reconstruction stencil just like the one used in ANSLib second-order least-squares reconstruction. These gradients are only used to reconstruct the solution value at the face mid-point (Gauss 
point) for pass two. Thus at the Gauss point we have

$$
\widetilde{\phi_{g}}=\phi_{L}+\nabla \phi_{L} \cdot \vec{r}_{L G}
$$

where $\vec{r}_{L G}$ is the distance from the cell centroid to the Gauss point location.

\subsubsection{Diffusion Gradients}

The second pass computes a distinct second gradient by applying the divergence theorem over the control volume.

$$
\iint_{C V_{i}} \nabla \phi d \mathcal{V}=\oint_{\partial C V_{i}} \phi \cdot \vec{A}
$$

which upon assuming the gradient $\nabla \phi$ to be constant over the control volume (first-order accurate) can be simplified as

$$
\nabla \phi_{C V_{i}} \Omega=\oint_{\partial C V_{i}} \phi \cdot \vec{A}
$$

where $\Omega$ is the size of the control volume (area in $2 \mathrm{D}$ and volume in $3 \mathrm{D}$ ).

In this divergence formulation, Fluent uses the averaged reconstructed solution values at the face. Thus the diffusion gradient for each control volume is formulated as

$$
\nabla \phi=\frac{1}{\Omega} \sum_{f}\left(\bar{\phi}_{g} \vec{A}\right)
$$

where $\vec{A}$ is the face area-vector and

$$
\bar{\phi}_{g}=\left(\frac{\widetilde{\phi_{g^{+}}}+\widetilde{\phi_{g^{-}}}}{2}\right) .
$$

This is a generalized formulation for both two and three dimensions, hence face-area vectors and volumes have been used.

Such a formulation extends the stencil that ultimately contributes to the formation of the gradients to include both the first and second layers of face-neighbours. 


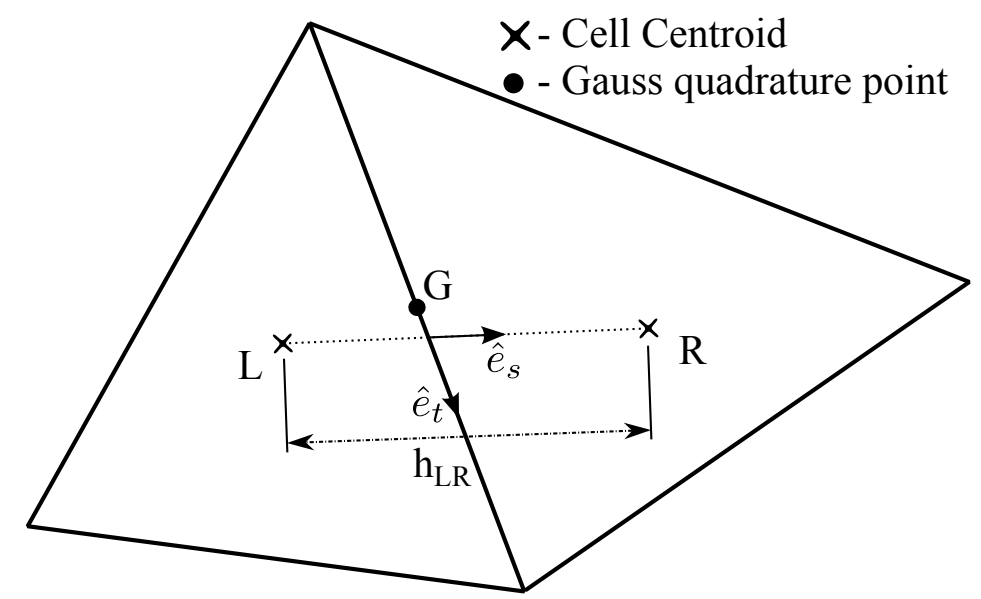

Figure 6.11: Illustration of Fluent diffusive flux terms

\subsubsection{Diffusive flux formulation}

The Fluent diffusive flux algorithm expresses the diffusion term at the face-centre

$$
\mathbb{F}_{D f}=\alpha(\nabla \phi \cdot \vec{A})
$$

where $\alpha$ is the diffusion coefficient, in terms of the solution values $(\phi)$ at the control-volume centroids of the left and right cell. The final diffusive flux formulation is given by

$$
\mathbb{F}_{D f}=\alpha\left(\frac{\phi_{L}-\phi_{R}}{h_{L R}}\right) \frac{\vec{A} \cdot \vec{A}}{\vec{A} \cdot \hat{e}_{s}}+\alpha\left(\overline{\nabla \phi} \cdot \vec{A}-\overline{\nabla \phi} \cdot \hat{e}_{s} \frac{\vec{A} \cdot \vec{A}}{\vec{A} \cdot \hat{e}_{s}}\right)
$$

where the averaged diffusion gradient of the two adjacent control volumes is used

$$
\overline{\nabla \phi}=\left(\frac{\nabla \phi_{L}+\nabla \phi_{R}}{2}\right)
$$

and $\hat{e}_{s}$ is the unit-normal vector along the line connecting the two control-volume centroids as depicted in Figure 6.11.

The extended stencil about the face $\mathrm{f}$ that results as a consequence of the diffusion gradient can be visualized in Figure 6.12a. In contrast a regular second-order least-squares flux discretization that was discussed in Section 2.1.3 would result in a first-layer faceneighbour stencil as illustrated in Figure 6.12b. 


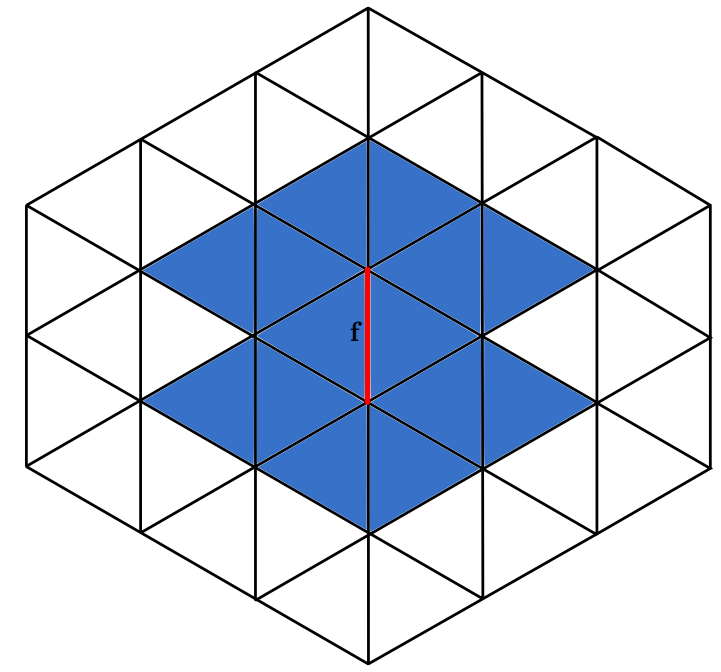

(a) Fluent scheme

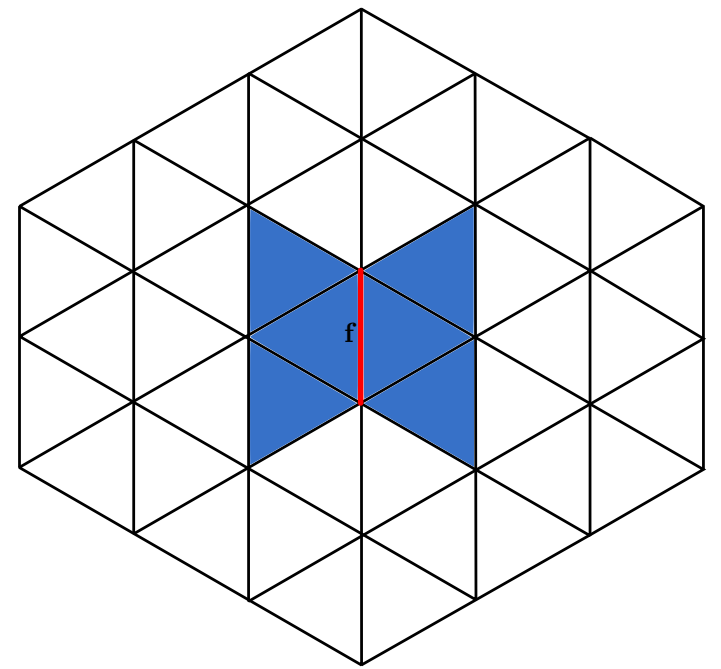

(b) Second-order least-squares scheme

Figure 6.12: Gradient stencils for diffusive fluxes about face $\mathrm{f}$

\subsubsection{Boundary Fluxes}

The boundary face centroids are considered as regular cell centroids at the boundaries and hence the diffusive flux takes a similar form as Equation 6.6 yielding

$$
\mathbb{F}_{D f}=\alpha\left(\frac{\phi_{L}-\phi_{b}}{h_{L b}}\right) \frac{\vec{A} \cdot \vec{A}}{\vec{A} \cdot \hat{e}_{b}}+\alpha\left(\nabla \phi_{L} \cdot \vec{A}-\nabla \phi_{L} \cdot \hat{e}_{b} \frac{\vec{A} \cdot \vec{A}}{\vec{A} \cdot \hat{e}_{b}}\right)
$$

where $\phi_{b}$ is the solution value at the boundary face and $\hat{e}_{b}$ is the unit-normal vector connecting the cell centroid and the boundary face centroid.

\subsection{2 $\quad \mathrm{H}_{1}$ Scheme in Fluent Diffusive Flux}

There are various design preferences and constraints to contend with when implementing changes within an existing algorithm or scheme. Keeping in mind these constraints, the following choices were made with regards to how we implement our scheme within theirs.

1. We retain the use of the modified $\mathrm{H}_{1}$ reconstruction which provides us with second order gradients since that was our motivation to begin with.

2. We use centroids instead of circumcentres/containment-centres since centroids are the accepted norm. 
3. We replace just their formulation of the diffusion gradients discussed in subsection 6.4.1.2 with the $\mathrm{H}_{1}$ flux discretization as

$$
\overline{\nabla \phi}_{H 1}=\frac{1}{\left(h_{L}+h_{R}\right)}\left\{h_{R} \cdot \nabla \phi_{L}+h_{L} \cdot \nabla \phi_{R}\right\}+O\left(h^{2}\right)
$$

where $\nabla \phi_{L}$ are the gradients obtained from the $\mathrm{H}_{1}$ reconstruction. The remaining formulation of the Fluent diffusive flux is maintained. Hence we have

$$
\mathbb{F}_{D f}=\alpha\left(\frac{\phi_{L}-\phi_{R}}{h_{L R}}\right) \frac{\vec{A} \cdot \vec{A}}{\vec{A} \cdot \hat{e}_{s}}+\alpha\left(\overline{\nabla \phi}_{H 1} \cdot \vec{A}-\overline{\nabla \phi}_{H 1} \cdot \hat{e}_{s} \frac{\vec{A} \cdot \vec{A}}{\vec{A} \cdot \hat{e}_{s}}\right)
$$

\subsubsection{Comparative Numerical Test Results}

Since we are dealing with two entirely different software packages that have very few things in common, to make any comparisons meaningful, we set up calibration tests. That involved getting the two codes, Fluent and ANSLib, to work on the same test cases as well as on the same meshes. The latter, as it turned out, was the tougher bottle-neck for which specific mesh converters had to be written. As for the test cases, the Poisson and advection-diffusion model problems were deemed sufficient and the problem set up was maintained exactly the same as those mentioned in Sections 5.2 and 5.3.

We then implemented the Fluent diffusion discretization into ANSLib and performed convergence studies, while keeping the reconstruction parameters such as inverse-distance weighting consistent in both pieces of software. Once satisfied with our calibrations, we ran comparison tests to assess the accuracy of various schemes including the $\mathrm{H}_{1}$ scheme within the Fluent discretization discussed in the previous section. The following finite-volume schemes were compared:

1. $\mathrm{H}_{1}$ finite-volume scheme (ANSLib-H1),

2. Second-order least-squares scheme with jump term (ANSLib- $\mathrm{A}_{2} \mathrm{~L}_{2}$-wJ),

3. Second-order least-squares scheme without jump term (ANSLib- $\mathrm{A}_{2} \mathrm{~L}_{2}$-nJ),

4. Fluent diffusive flux discretization in ANSLib (ANSLib-Fluent), and 


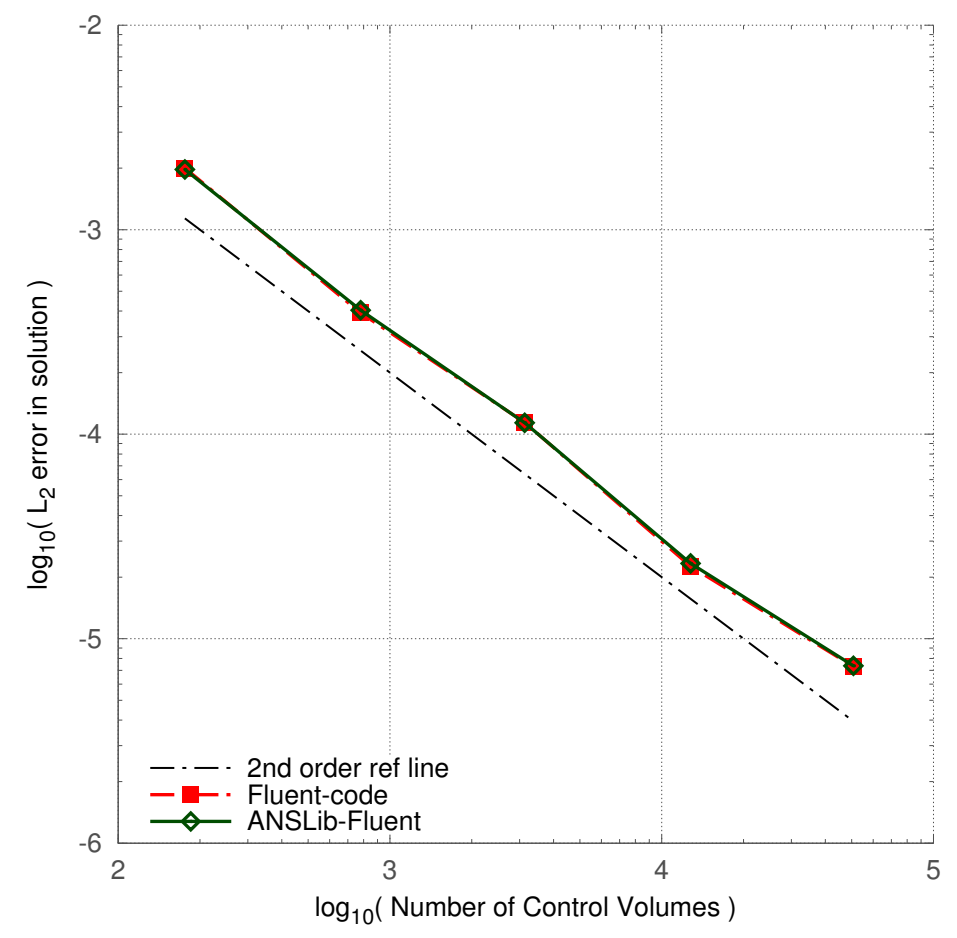

Figure 6.13: Poisson solution convergence rates in Fluent software (Fluent-code) and Fluent discretization implementation in ANSLib (ANSLib-Fluent)

5. $\mathrm{H}_{1}$ scheme in Fluent diffusive flux (ANSLib-H1Fluent)

The results of these calibration tests as well as the full comparison tests are presented in the following subsections.

\subsubsection{2D Poisson Test}

The Poisson test setup is similar to the one used in Section 5.2, wherein the test function $\frac{\pi}{8} \sin (\pi x) \sin (\pi y)$ and associated source term was used for the method of manufactured solutions along with homogeneous Dirichlet boundary conditions and smooth initial solution data. Figure 5.3 shows the resultant manufactured solution on a unit square mesh consisting of 780 triangles.

Figure 6.13 shows how the Fluent discretization implementation in ANSLib fares compared to the actual Fluent software. The Poisson solution accuracy convergence rates have been plotted and as can be seen they overlap each other. Thus we can be satisfied with the comparative studies that follow, which only plot the ANSLib-Fluent discretization for 


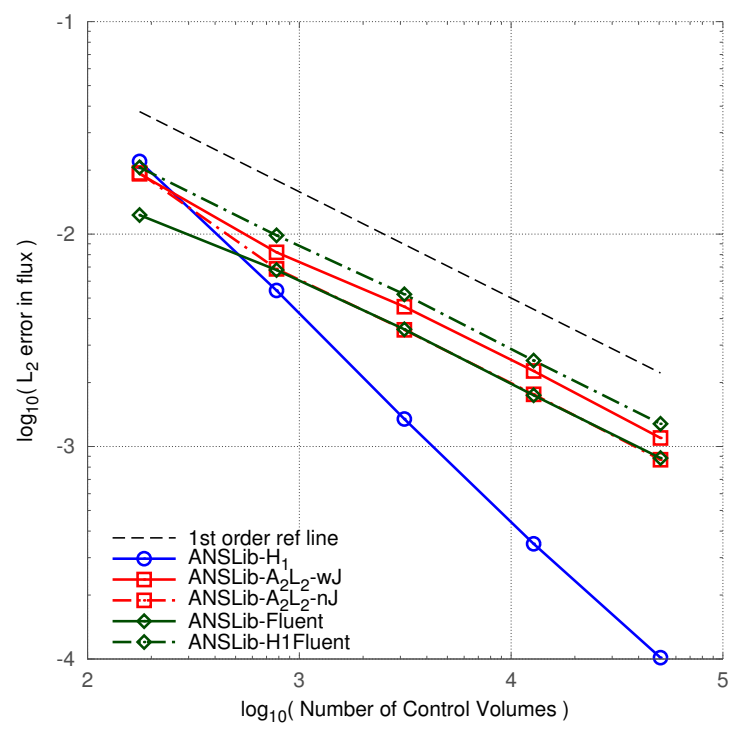

(a) Flux convergence rate at Gauss points (initial data)

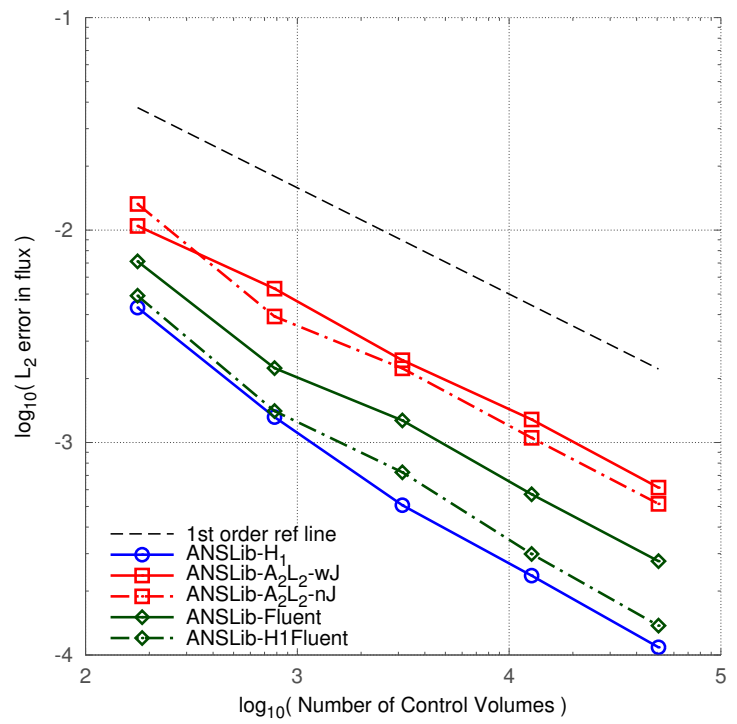

(c) Flux convergence rate at Gauss points (converged solution)

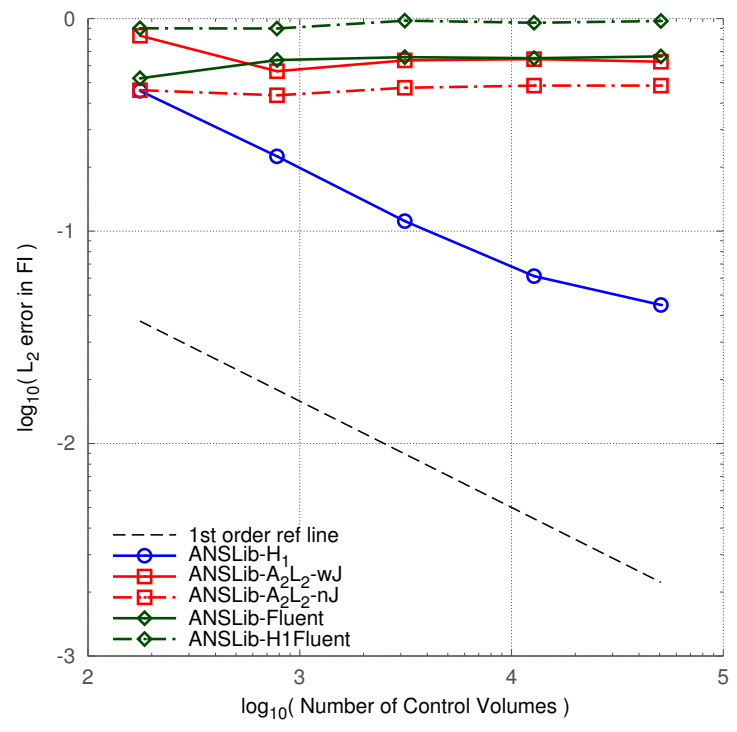

(b) Flux integral convergence rate (initial data)

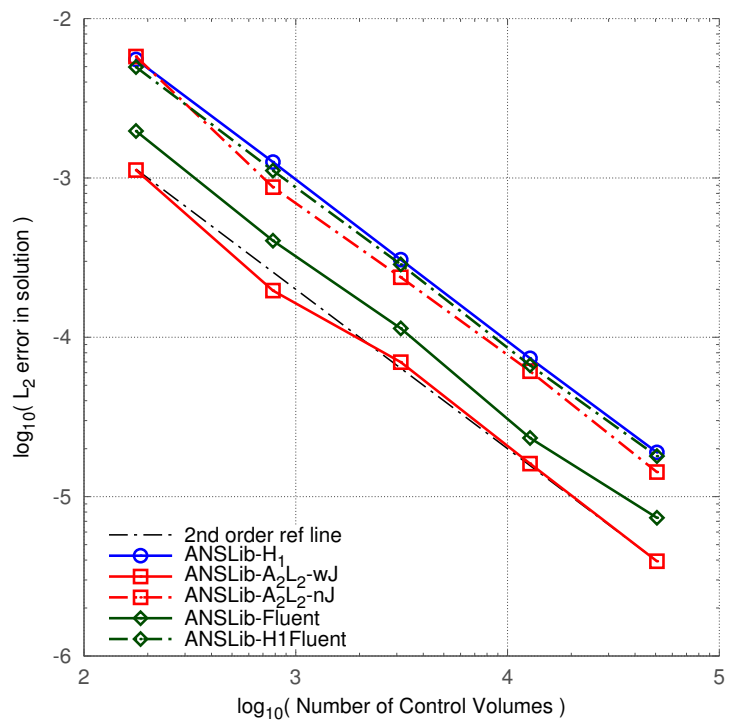

(d) Solution convergence rate

Figure 6.14: Poisson test results 
simplicity.

The asymptotic convergence of the diffusive flux obtained using smooth initial data has been shown in Figure 6.14a. We observe that the full $\mathrm{H}_{1}$ reconstruction flux, with secondorder accuracy, does better than the rest of the schemes, which are first-order accurate. However our $\mathrm{H}_{1}$ scheme in the Fluent discretization does not fare well, because cell centroids are used instead of circumcentres. Moreover, the additional terms from Equation 6.10 present in the $\mathrm{H}_{1}$-Fluent flux, apart from the $\mathrm{H}_{1}$ gradients, may be adversely affecting the flux accuracy. A similar trend can be seen in the flux integral accuracy in Figure 6.14b.

Following that result, we can observe the flux accuracy at the end of complete solution convergence in Figure 6.14c. All the schemes here have significantly smaller error than when using the exact initial solution (Figure 6.14a). The full $\mathrm{H}_{1}$ reconstruction flux is no longer second order in the presence of the solution error, though is is still the most accurate. In contrast, the $\mathrm{H}_{1}$-Fluent discretization performs much better relative to other schemes.

All the more interesting is the plot of the converged solution accuracies seen in Figure $6.14 \mathrm{~d}$ where we can observe that the rankings have almost been reversed save for the second-order least-squares with jump term which does exceptionally well.

\subsubsection{2D Advection-Diffusion Test}

Once again we set up the same advection-diffusion case that was described in Section 5.3, with an unstructured rectangular-channel mesh.

The ANSLib-Fluent implementation does compare well enough with the results from the Fluent software as we can see in Figure 6.15. However, variations arise in the advection dominated flow $(\alpha=0.01)$ which indicates that the advection flow solution for the two do not quite match up. The advection solution and its corresponding flux are heavily dependent on the solution reconstruction, which is identical in the interior. Hence we can infer that the second-order least-squares reconstruction used in the two software packages vary slightly at the boundaries. Nonetheless, the two results were deemed to be close enough to move ahead with our comparisons.

The full comparison test plots for the convergence of the advection-diffusion solution have been presented in Figure 6.16. $\mathrm{H}_{1}$ finite-volume is slightly more accurate than the other 


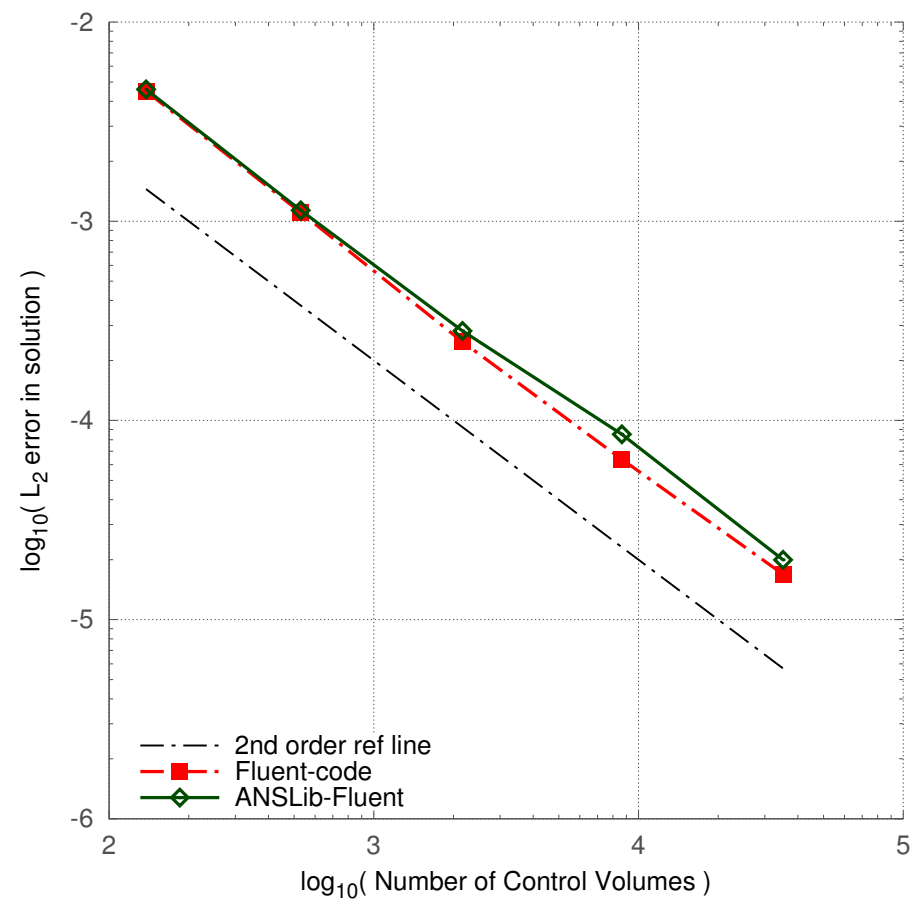

Diffusion Dominant $(\alpha=1)$

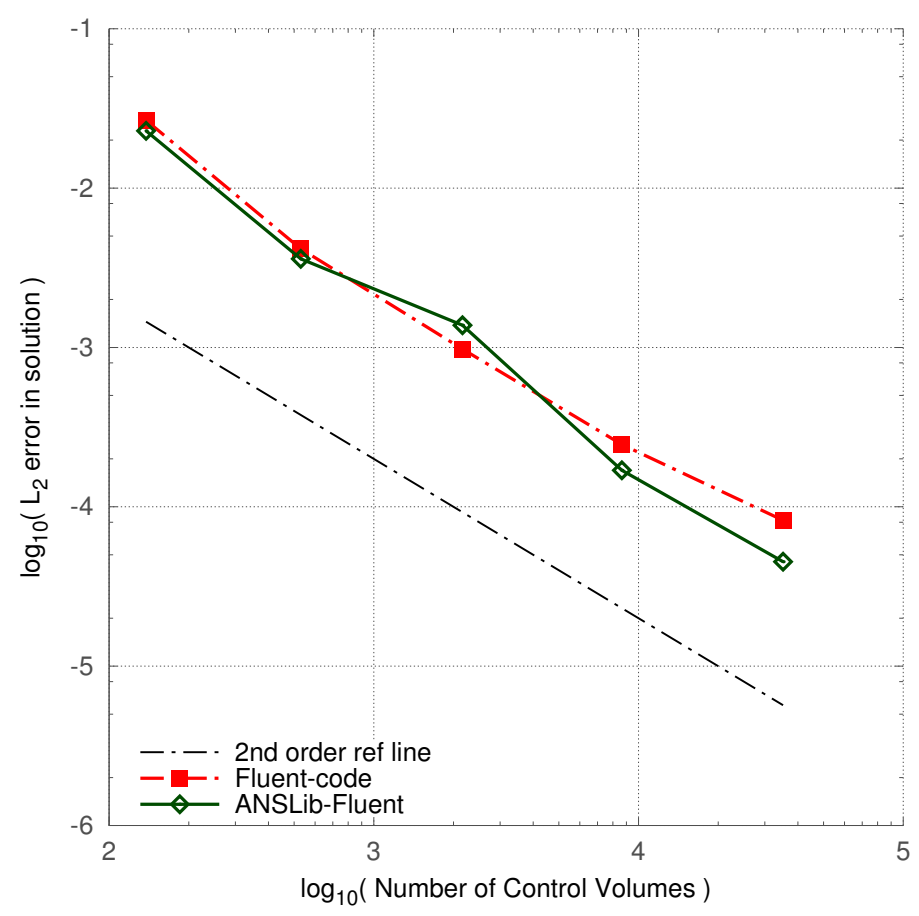

Advection Dominant $(\alpha=0.01)$

Figure 6.15: Advection-Diffusion solution convergence rates in Fluent software and Fluent discretization implementation in ANSLib 


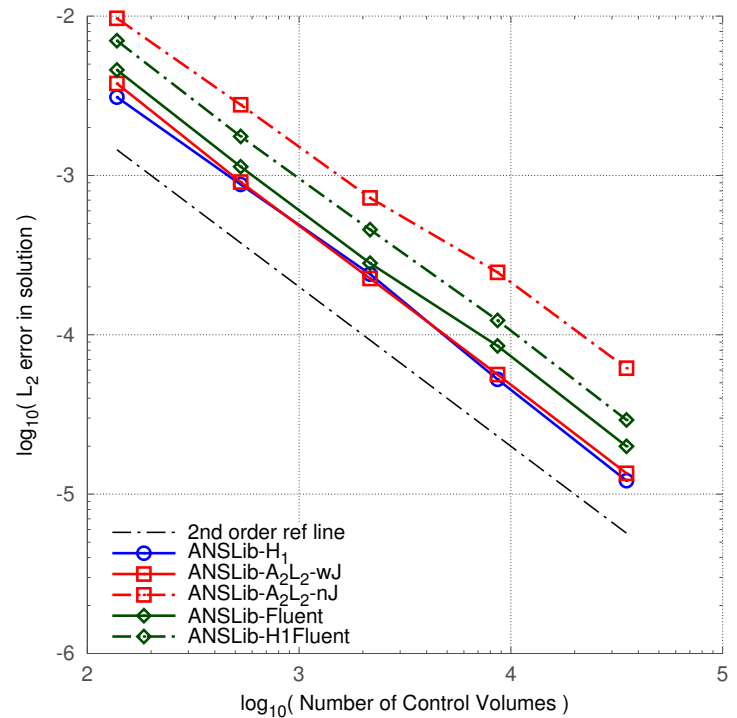

Diffusion Dominant $(\alpha=1)$

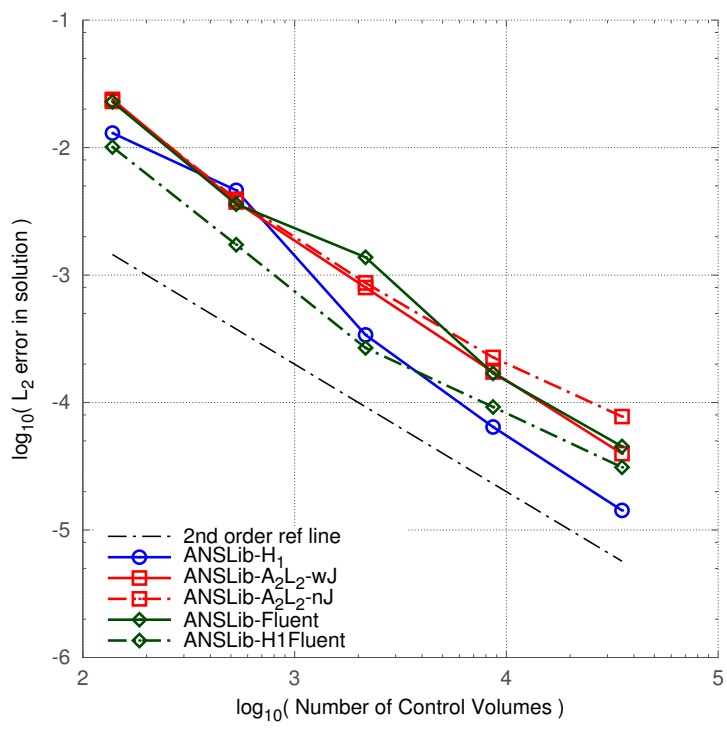

Advection Dominant $(\alpha=0.01)$

Figure 6.16: Advection-Diffusion Solution convergence rates

schemes, while the $\mathrm{H}_{1}$-Fluent implementation does well in the advection dominated case. The convective flux seems to have a positive influence on the $\mathrm{H}_{1}$-Fluent implementation. The second-order least-squares without jump term scheme does not perform as well as the others in both the cases.

The presence of the upwind convective flux in the advection-diffusion problem can be seen as a positive influence on the stability and accuracy of the $\mathrm{H}_{1}$ finite-volume scheme as we saw in Sections 6.1 and 5.3. However in the case of the $\mathrm{H}_{1}$-Fluent scheme, once again the influence of the unmodified terms in Equation 6.10 seems to have significant effects on the solution. 


\section{Chapter 7}

\section{Summary and Conclusions}

Any implementation of the finite-volume gradients has always been expected to be an order less accurate than the solution itself. This applies to the diffusive fluxes as well as any other quantity derived from the gradients. The research described in this thesis was aimed at addressing that concern and we have presented a solution. In the following sections we will summarize, highlight the major conclusions and also present our visions for the future expansions of the research described in this thesis.

\section{$7.1 \quad$ Summary}

A novel finite-volume scheme to evaluate the diffusive fluxes to second-order accuracy was developed and presented in this thesis. It has the potential to improve their accuracy, raising it by an entire order even on unstructured meshes and thus counter the loss of an order in comparison to the solution accuracy. A more accurate diffusive flux should cascade into improvements in the flux integral and this has been shown to be true with our results. A finite-volume formulation was utilized as the foundation to develop the new scheme since it is currently widely implemented for practical application in the industry. Although more accurate high-order schemes have been around for a while, they have not gained traction in the industry due to the higher computational complexity involved and lack of robustness. Hence the industry standard is only second-order accuracy. This scheme was developed to bridge that gap, among other benefits including a better truncation error estimation.

The framework of a general least-squares finite-volume scheme was described in detail. The proposed $\mathrm{H}_{1}$ reconstruction procedure was then described in relation to a second and third-order least-squares scheme, to provide second-order gradients and without evaluating the second-degree derivatives to save on overall computational resources utilized. The 
circumcentres were used as the primary control-volume reference points, with containmentcentres being implemented only when adjacent triangles had nearly coincident circumcentres. Second-order diffusive fluxes are then computed from these gradient values without the use of second-degree derivatives using a linear interpolation flux discretization. The flux integral is obtained from these flux estimates using a single-point Gauss quadrature. We also introduce a high-accuracy jump term to stabilize the scheme yet not degrade its asymptotic accuracy.

Rigorous tests were conducted to verify the proposed scheme as well as to ascertain its characteristics and behaviour. Both analytical and numerical approaches were used as verification procedures.

The analytical approach gave us preliminary insights into the accuracy of the scheme on a uniform equilateral triangle grid. On this grid, the computations can be handled analytically with the aid of symbolic algebra software tools. We analyzed and tweaked the reconstruction stencil used in our scheme as well as studying the resulting error in the Laplacian (truncation error) while comparing with the second and third-order least-squares schemes. We were also able to study the decoupling phenomenon that can been seen in second-order least-squares as well as $\mathrm{H}_{1}$ reconstruction schemes.

The numerical approach on the other hand is not restricted by the number of control volumes and their topology. Hence it is ideal to study the scheme on unstructured meshes. Preliminary tests were conducted to verify the foundations of our scheme's linear interpolation flux discretization in one dimension as well as the $\mathrm{H}_{1}$ reconstruction accuracy in two dimensions. The Poisson model equation was used as the primary test case to isolate and analyze the diffusive flux. The Method of Manufactured Solutions (MMS), with a set of three exact solutions for Poisson's equation, was used to evaluate the discretization error and study the convergence characteristics of the solution, the flux and the flux integral. The expected convergence was verified and compared with those from the least-squares schemes. The effect of the two alternative implementations of control-volume reference points was studied. The implementation and verification of $\mathrm{H}_{1}$ finite-volume scheme in the advection-diffusion model problem was also studied.

Once satisfied with the accuracy and implementation of the scheme, we analyzed its 
various characteristics and behaviour, concentrating on its stability, truncation error and resource usage. The stability analysis was visualized by studying the eigenvalues spectrum along the lines of the eigenanalysis strategy proposed by Ollivier-Gooch and Roy [42]. We were able to gain much insight into the effect that choice of control-volume reference points and the use of a jump term had on the overall stability of different schemes. A truncation error analysis of our scheme was also performed using methods proposed by Jalali and Ollivier-Gooch [20]. We compared the truncation error of our scheme with that of second and third-order least-squares schemes. Finally an in-depth cost analysis was conducted to verify the advantages gained over other least-squares schemes and possible avenues of improvements.

The behaviour of $\mathrm{H}_{1}$ finite-volume scheme within the Fluent diffusive flux discretization scheme was also explored. The Fluent diffusive discretization was implemented within ANSLib and baseline tests were performed to ensure the implementation worked as well as the actual Fluent software itself. Several schemes and their variations were then compared on the chosen model problems of Poisson and advection-diffusion to study and compare the behaviour of each.

\subsection{Conclusions}

Although the research and development of a new finite-volume scheme is challenging and inevitably fraught with unforeseen complications, we are happy to be able to present one which does fulfil the expectations.

By means of the analytical tests we were able to verify the reconstruction order of accuracy for linear (second-order) least-squares reconstruction on cell-centred uniform equilateral triangle meshes. However we do observe inconsistencies in the quadratic reconstruction which has a leading-order-error term which is first-order resulting from degeneracy in reconstruction on uniform triangular mesh. $\mathrm{H}_{1}$ reconstruction being a derivative of the quadratic reconstruction, seems to inherit this inconsistency along with an unforeseen solution decoupling unique to $\mathrm{H}_{1}$ reconstruction.

Fortunately the numerical tests, conducted with cell-centred triangular unstructured 
meshes on Poisson and advection-diffusion problems, show more promising results. An overview of the results convey that the diffusive fluxes do indeed have an improved order of accuracy while the solution itself is more accurate than second-order least-squares scheme by a constant factor, albeit a small factor. The flux integral at zero iterations, which has much smaller error, shows much improvement in order for the case where we replace only the "ugly circumcentres" with containment centres. In addition, the solution error is also much smoother and lies between that of second and third order least-squares schemes in terms of smoothness.

The complication of stability was by far the toughest hurdle to overcome with very few options available that would not adversely sacrifice the gains in accuracy of the diffusive flux. The use of circumcentres as the control-volume reference points turned out to be a highly unstable choice. The solution presented itself in the form of containment centres and more importantly the high-accuracy jump term.

The truncation error again showed some improvement compared to the second-order least-squares reconstruction, although not as much as we would have wished for. The high and low frequency modes of the eigenvalues appear to share similar magnitude in the truncation error for $\mathrm{H}_{1}$ reconstruction.

As for computational resource usage, as anticipated, $\mathrm{H}_{1}$ reconstruction is cheaper than a full third-order least-squares reconstruction. The Jacobian evaluation time is twice as fast as the third-order scheme and almost on par with the second-order scheme. However the memory requirement is identical to the third-order scheme. This is expected since the memory requirement is essentially only dependent on the number of neighbours in the reconstruction stencil and $\mathrm{H}_{1}$ reconstruction uses the exact same stencil as the third-order scheme. These results also translate into the total wall convergence time per iteration since the Jacobian evaluation time is the largest single part of the computational time. However at the moment $\mathrm{H}_{1}$ reconstruction takes a couple more iterations to converge than either of the least-squares schemes and this adversely affects the overall time taken for convergence.

The study of the Fluent diffusive flux discretization was a very insightful experience. Every discretization scheme has its unique advantages and disadvantages, so comparing these idiosyncrasies and their causes is overall always beneficial. For instance, the extended 
stencil that results from the use of the distinct Fluent diffusion gradients and its inferred effect on the stability of the scheme was enlightening. The implementation of the Fluent discretization into ANSLib was completely successful and the matching results reinforced the similarities of other underlying algorithms. However combining the $\mathrm{H}_{1}$ scheme with the Fluent discretization provided mixed success that was not entirely as we anticipated. The seemingly inverse relation between the flux accuracy and solution accuracy convergence for the Poisson scheme was the highlight of those results and its cause will be probed further in the near future.

In conclusion, looking at the whole picture, the proposed new $\mathrm{H}_{1}$ finite-volume scheme meets our research objectives of striking a balance between the best of second and third order finite-volume schemes.

\subsection{Recommended Future Work}

Our work with the $\mathrm{H}_{1}$ finite-volume scheme paves the way for further analysis, extensions and applications.

1. Work can be geared towards improving the convergence characteristics as well as improving the robustness of the scheme.

2. The current research has only focused on the interior regions of the mesh. Implementation in the boundary regions is another important step. The boundary discretization is the most challenging part of any numerical scheme and thus the impact of the boundaries on the solution and its gradients cannot be neglected. A possible route to the same could be through the effective usage of ghost cells.

3. We could also look at possible implementations for vertex-centred meshes. Vertexcentred mesh discretizations have the advantage of being slightly more robust than their cell-centred counterparts due to more numerous first neighbours, as well as compact stencils for third-order (and hence for $\mathrm{H}_{1}$ reconstruction).

4. The present research was limited to second-order solution and gradients. The extension to higher order would be more challenging yet potentially lucrative. The 
anticipated challenge would be dealing with the two Gauss point values required for higher order accuracy.

5. Extension from two dimensions to three dimensions is another avenue to look at. Using circumcentres of tetrahedrons should be straightforward. However handling issues of stability in three dimensions could be a significant challenge.

6. Future explorations could include extension from simple model problems to the more complex real fluid flows defined by the Navier-Stokes equations, possibly in combination with existing viscous flux discretization schemes, and study of their combined characteristics.

7. Another area of future work is application to problems with gradient-based source terms, a class which includes the production term for many RANS turbulence models including the Spalart-Allmaras turbulence models $[53,52]$. The requirements for improved accuracy in source term evaluation and its impact on solution accuracy will be of primary interest. 


\section{Bibliography}

[1] Rémi Abgrall. On essentially non-oscillatory schemes on unstructured meshes: Analysis and implementation. Journal of Computational Physics, 114(1):45-58, 1994.

[2] John D. Anderson. Computational Fluid Dynamics: The Basics with Applications. McGraw-Hill Education, 1995.

[3] Timothy J. Barth. Aspects of unstructured grids and finite-volume solvers for the Euler and Navier-Stokes equations. In Unstructured Grid Methods for Advection-Dominated Flows, pages 6-1 - 6-61. AGARD, Neuilly sur Seine, France, 1992. AGARD-R-787.

[4] Timothy J. Barth. Recent developments in high order k-exact reconstruction on unstructured meshes. AIAA paper 93-0668, January 1993.

[5] Timothy J. Barth and Herman Deconinck. High-order methods for computational physics, volume 9. Springer Science \& Business Media, 1999.

[6] Timothy J. Barth and Paul O. Frederickson. Higher order solution of the Euler equations on unstructured grids using quadratic reconstruction. AIAA paper 90-0013, January 1990.

[7] Timothy J. Barth and Dennis C. Jespersen. The design and application of upwind schemes on unstructured meshes. AIAA paper 89-0366, January 1989.

[8] Timothy J. Barth and Mario Ohlberger. Finite Volume Methods: Foundation and Analysis. John Wiley \& Sons, Ltd, 2004.

[9] Alexander N. Brooks and Thomas J. R. Hughes. Streamline upwind/Petrov-Galerkin formulations for convection dominated flows with particular emphasis on the incom- 
pressible Navier-Stokes equations. Computer methods in applied mechanics and engineering, 32(1):199-259, 1982.

[10] Bernardo Cockburn and Chi-Wang Shu. Runge-Kutta discontinuous Galerkin methods for convection-dominated problems. Journal of scientific computing, 16(3):173-261, 2001.

[11] Boris Diskin, James L. Thomas, Eric J. Nielsen, Hiroaki Nishikawa, and Jeffrey A. White. Comparison of node-centered and cell-centered unstructured finite-volume discretizations: Viscous fluxes. American Institute of Aeronautics and Astronautics Journal, 48(7):1326-1338, July 2010.

[12] Olivier Friedrich. Weighted essentially non-oscillatory schemes for the interpolation of mean values on unstructured grids. Journal of Computational Physics, 144(1):194-212, July 1998.

[13] Michael B. Giles and Niles A. Pierce. An introduction to the adjoint approach to design. Flow, Turbulence, and Combustion, 65(3-4):393-415, 2000.

[14] Ami Harten, Björn Enquist, Stanley Osher, and Sukumar R. Chakravarthy. Uniformly high order accurate essentially non-oscillatory schemes, III. Journal of Computational Physics, 71(2):231-303, August 1987.

[15] Jan S. Hesthaven and Tim Warburton. Nodal discontinuous Galerkin methods: algorithms, analysis, and applications. Springer Science \& Business Media, 2007.

[16] Thomas J. R. Hughes, Michel Mallet, and Mizukami Akira. A new finite element formulation for Computational Fluid Dynamics: II. beyond SUPG. Computer Methods in Applied Mechanics and Engineering, 54(3):341-355, 1986.

[17] H.T. Huynh. A flux reconstruction approach to high-order schemes including discontinuous Galerkin methods. AIAA paper, 4079:2007, 2007.

[18] H.T. Huynh, Z. J. Wang, and P.E. Vincent. High-order methods for computational fluid dynamics: A brief review of compact differential formulations on unstructured grids. Computers \&f Fluids, 98:209-220, 2014. 
[19] Alireza Jalali. Truncation error analysis of unstructured finite volume discretization schemes. Master's thesis, The University of British Columbia, Department of Mechanical Engineering, 2012.

[20] Alireza Jalali and Carl Ollivier-Gooch. Accuracy assessment of finite volume discretizations of diffusive fluxes on unstructured meshes. In Proceedings of the Fiftieth AIAA Aerospace Sciences Meeting. American Institute of Aeronautics and Astronautics, 2012. AIAA Paper 2012-0608.

[21] Alireza Jalali, Mahkame Sharbatdar, and Carl Ollivier-Gooch. Accuracy analysis of unstructured finite volume discretization schemes for diffusive fluxes. Computers 86 Fluids, 101:220-232, 2014.

[22] Aaron Katz and Venkateswaran Sankaran. An efficient correction method to obtain a formally third-order accurate flow solver for node-centered unstructured grids. Journal of Scientific Computing, 51(2):375-393, 2012.

[23] David A. Kopriva and John H. Kolias. A conservative staggered-grid Chebyshev multidomain method for compressible flows. Journal of Computational Physics, 125(1):244 $-261,1996$.

[24] Sanjiva K. Lele. Compact finite difference schemes with spectral-like resolution. Journal of computational physics, 103(1):16-42, 1992.

[25] Xu-Dong Liu, Stanley Osher, and Tony Chan. Weighted essentially non-oscillatory schemes. Journal of Computational Physics, 115:200-212, 1994.

[26] Yen Liu, Marcel Vinokur, and ZJ Wang. Spectral difference method for unstructured grids i: basic formulation. Journal of Computational Physics, 216(2):780-801, 2006.

[27] Harvard Lomax, Thomas H. Pulliam, and David W. Zingg. Fundamentals of Computational Fluid Dynamics. Springer Science \& Business Media, 2001.

[28] S. R. Mathur and J. Y. Murthy. A pressure-based method for unstructured meshes. Numerical Heat Transfer, 31(2):195-215, 1997. 
[29] Dimitri J. Mavriplis. Revisiting the least-squares procedure for gradient reconstruction on unstructured meshes. In Proceedings of the Sixteenth AIAA Computational Fluid Dynamics Conference, 2003.

[30] Christopher Michalak. Efficient high-order accurate unstructured finite-volume algorithms for viscous and inviscid compressible flows. $\mathrm{PhD}$ thesis, The University of British Columbia, Department of Mechanical Engineering, 2009.

[31] Christopher Michalak and Carl Ollivier-Gooch. Globalized matrix-explicit NewtonGMRES for the high-order accurate solution of the Euler equations. Computers and Fluids, 39:1156-1167, 2010.

[32] Amir Nejat. A Higher-Order Accurate Unstructured Finite Volume Newton-Krylov Algorithm for Inviscid Compressible Flows. PhD thesis, The University of British Columbia, Department of Mechanical Engineering, 2007.

[33] Amir Nejat and Carl Ollivier-Gooch. A high-order accurate unstructured finite volume Newton-Krylov algorithm for inviscid compressible flows. Journal of Computational Physics, 227(4):2592-2609, 2008.

[34] Hiroaki Nishikawa. A first-order system approach for diffusion equation. I: Secondorder residual-distribution schemes. Journal of Computational Physics, 227(1):315$352,2007$.

[35] Hiroaki Nishikawa. Beyond interface gradient: A general principle for constructing diffusion schemes. In Proceedings of the Fortieth AIAA Fluid Dynamics Conference, 2010.

[36] Hiroaki Nishikawa. A first-order system approach for diffusion equation. II: Unification of advection and diffusion. Journal of Computational Physics, 229(11):3989-4016, 2010.

[37] Carl Ollivier-Gooch, Amir Nejat, and Christopher Michalak. On obtaining and verifying high-order finite-volume solutions to the Euler equations on unstructured meshes. American Institute of Aeronautics and Astronautics Journal, 47(9):2105-2120, 2009. 
[38] Carl F. Ollivier-Gooch. High-order ENO schemes for unstructured meshes based on least-squares reconstruction. AIAA paper 97-0540, January 1997.

[39] Carl F. Ollivier-Gooch. Quasi-ENO schemes for unstructured meshes based on unlimited data-dependent least-squares reconstruction. Journal of Computational Physics, 133(1):6-17, 1997.

[40] Carl F. Ollivier-Gooch. An unstructured mesh improvement toolkit with application to mesh improvement, generation and (de-)refinement. AIAA 98-0218, January 1998.

[41] Carl F. Ollivier-Gooch. A toolkit for numerical simulation of PDE's: I. Fundamentals of generic finite-volume simulation. Computer Methods in Applied Mechanics and Engineering, 192:1147-1175, February 2003.

[42] Carl F. Ollivier-Gooch and Christopher Roy. Reducing truncation error on unstructured meshes by vertex movement. In Proceedings of the Forty-Second AIAA Fluid Dynamics Conference, 2012.

[43] Carl F. Ollivier-Gooch and Michael Van Altena. A high-order accurate unstructured mesh finite-volume scheme for the advection-diffusion equation. Journal of Computational Physics, 181(2):729-752, 2002.

[44] B. Pincock and A. Katz. High-order flux correction for viscous flows on arbitrary unstructured grids. Journal of Scientific Computing, 61(2):454-476, 2014.

[45] Thomas H Pulliam and Joseph L Steger. Implicit finite-difference simulations of threedimensional compressible flow. AIAA Journal, 18(2):159-167, 1980.

[46] Varun P. Puneria. Truncation error analysis for diffusion schemes in boundary regions of unstructured meshes. Master's thesis, The University of British Columbia, Department of Mechanical Engineering, 2015.

[47] Varun P. Puneria and Carl Ollivier-Gooch. Truncation error analysis for diffusion schemes in boundary regions of unstructured meshes. In Proceedings of the TwentySecond Annual Conference of the Computational Fluid Dynamics Society of Canada. Société canadienne de CFD / CFD Society of Canada, 2014. 
[48] Christopher Roy. Strategies for driving mesh adaptation in CFD. In Proceedings of the Forty-Seventh AIAA Aerospace Sciences Meeting. American Institute of Aeronautics and Astronautics, 2009.

[49] Christopher Roy. Review of discretization error estimators in scientific computing. In Proceedings of the Forty-Eighth AIAA Aerospace Sciences Meeting. American Institute of Aeronautics and Astronautics, 2010. AIAA Paper 2010-0126.

[50] Mahkame Sharbatdar and Carl Ollivier-Gooch. Smooth estimate of the truncation error for unstructured mesh finite volume methods. In Proceedings of the Sixth European Conference on Computational Fluid Dynamics, 2014.

[51] Chi-Wang Shu. High-order finite difference and finite volume WENO schemes and discontinuous galerkin methods for CFD. International Journal Computational Fluid Dynamics, 17:107-118, 2003.

[52] P. R. Spalart. Strategies for turbulence modelling and simulations. International Journal of Heat and Fluid Flow, 21(3):252 - 263, 2000.

[53] P. R. Spalart and S. R. Allmaras. A one-equation turbulence model for aerodynamic flows. In Proceedings of the Thirtieth AIAA Aerospace Sciences Meeting, January 1992.

[54] James L. Thomas, Boris Diskin, and Christopher Rumsey. Towards verification of unstructured-grid solvers. In Forty-sixth AIAA Aerospace Sciences Meeting, 2008. AIAA 2008-666.

[55] Michael Van Altena. High-order finite-volume discretisations for solving a modified advection-diffusion problem on unstructured triangular meshes. Master's thesis, The University of British Columbia, Department of Mechanical Engineering, October 1999.

[56] V. Venkatakrishnan. A perspective on unstructured grid flow solvers. Technical report, ICASE 95-3 NASA, 1995.

[57] Henk Kaarle Versteeg and Weeratunge Malalasekera. An introduction to computational fluid dynamics: the finite volume method. Pearson Education, 2007. 
[58] P. Vincent, P. Castonguay, and A. Jameson. A new class of high-order energy stable flux reconstruction schemes. Journal of Scientific Computing, 47(1):50-72, 2011.

[59] Z. J. Wang, Krzysztof Fidkowski, Rémi Abgrall, Francesco Bassi, Doru Caraeni, Andrew Cary, Herman Deconinck, Ralf Hartmann, Koen Hillewaert, HT Huynh, et al. High-order CFD methods: current status and perspective. International Journal for Numerical Methods in Fluids, 72(8):811-845, 2013.

[60] Gary Kai-Kin Yan, Varun P. Puneria, Alireza Jalali, and Carl Ollivier-Gooch. Truncation and discretization error for diffusion schemes on unstructured meshes. In Proceedings of the Fifty-Second AIAA Aerospace Sciences Meeting. American Institute of Aeronautics and Astronautics, 2014. 